\title{
Understanding Chemoselectivity in Proton-Coupled Electron Transfer: A Kinetic Study of Amide and Thiol Activation
}

Guanqi Qiu and Robert R. Knowles*

Department of Chemistry, Princeton University, Princeton, NJ 08544, USA

\section{Supporting Information}

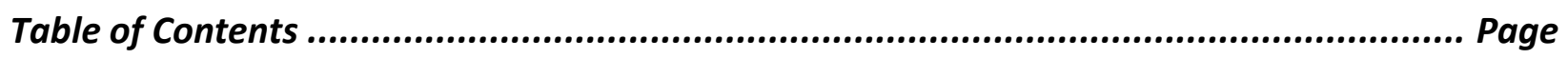

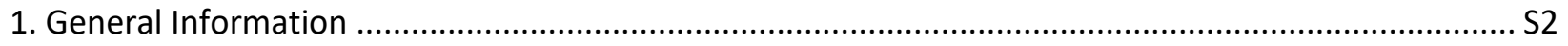

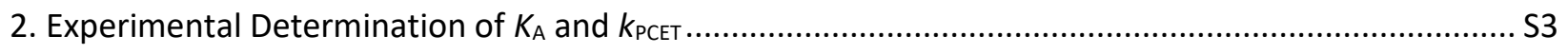

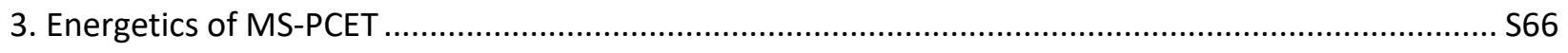

4. Synthesis and Characterization of Substrates and Products ............................................................ S67

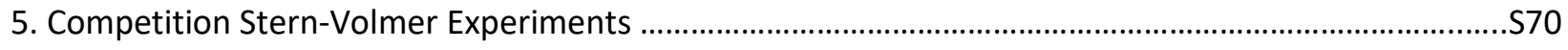

6. Calculation of MS-PCET Selectivity Ratio $Q_{0}$ in Catalytic Hydroamidation Reactions............................. S77

7. Deconvolution of the Effect from Thiol-Related Steps Downstream in the Catalytic Cycle ................. S91

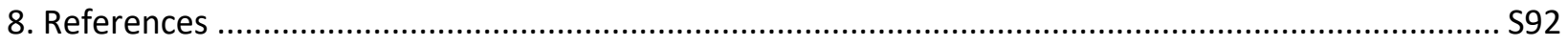




\section{General Information}

Commercial reagents were purified prior to use following the guidelines of Perrin and Armarego. ${ }^{1}$ All solvents were purified and stored according to the method of Grubbs. ${ }^{2}$ All luminescence quantities were measured using steady-state Stern-Volmer experiments on an Agilent Cary Eclipse fluorescence spectrophotometer. Stern-Volmer fluorescence quenching experiments were run with freshly prepared (typically $5 \times 10^{-6} \mathrm{M}$ ) solutions of $\operatorname{Ir}(\mathrm{III})$ photocatalyst in 1,2-dichloroethane (DCE) at ambient temperature $\left(22-24{ }^{\circ} \mathrm{C}\right)$ under an argon atmosphere. All solutions were degassed with argon before luminescence measurement. The irradiation and emission wavelengths depend on the specific photocatalyst used. In all Stern-Volmer experiments, the fluorescence intensity was measured three times for each sample. Timeresolved luminescence experiments were used qualitatively to identify the nature (static or dynamic) of the relevant steady-state quenching experiments. These experiments were conducted using timecorrelated single-photon counting with Horiba DeltaFlex.

${ }^{1} \mathrm{H}$ and ${ }^{13} \mathrm{C}$ NMR spectra were recorded on a Bruker 500 (500 and $126 \mathrm{MHz}$ for ${ }^{1} \mathrm{H}$ and ${ }^{13} \mathrm{C}$ respectively) instrument, and are internally referenced to residual solvent signals, $\mathrm{CDCl} 3$ referenced at $\delta 7.26$ and 77.16 ppm. Data for $1 \mathrm{H}$ is reported as follows: chemical shift $(\delta \mathrm{ppm})$, integration, multiplicity $(\mathrm{s}=$ singlet, $\mathrm{d}=$ doublet, $\mathrm{t}=$ triplet, $\mathrm{q}=$ quartet, $\mathrm{m}=$ multiplet), broad peaks $(\mathrm{br})$, coupling constant $(\mathrm{Hz})$ and assignment. Dimethyl sulfone was used as the internal standard to determine the NMR yield. IR spectra were recorded on a Perkin Elmer Paragon 1000 spectrometer and are reported in terms of frequency of absorption $\left(\mathrm{cm}^{-1}\right)$. High-resolution mass spectra were obtained at Princeton University mass spectrometry facilities using an Agilent 6210 TOF LC/MS. 


\section{Experimental Determination of $K_{\mathrm{A}}$ and $\boldsymbol{k}_{\mathrm{PCET}}$}

Table S2. Kinetic and H-bonding equilibrium data for ketone MS-PCET reactions

\begin{tabular}{|c|c|c|c|c|c|c|}
\hline entry & $\begin{array}{l}\text { amide } \\
\text { /thiol }\end{array}$ & $* \operatorname{Ir}(\mathrm{III})$ & $\begin{array}{c}\mathrm{BDFE}_{\mathrm{N}-\mathrm{H} / \mathrm{S}-\mathrm{H}}{ }^{\text {ref }} \\
(\mathrm{kcal} / \mathrm{mol})\end{array}$ & $K_{\mathrm{A}}\left(\mathrm{M}^{-1}\right)$ & $\begin{array}{l}\Delta \mathrm{G}^{\circ}{ }^{\prime} \mathrm{PCET} \\
(\mathrm{kcal} / \mathrm{mol})\end{array}$ & $\begin{array}{c}k_{\mathrm{PCET}} \\
\left(\mathrm{M}^{-1} \mathrm{~s}^{-1}\right)\end{array}$ \\
\hline 1 & Al & $|r-|$ & 98.9 & 1050 & 8.4 & $8.4 \times 10^{9}$ \\
\hline 2 & Al & Ir-2 & 98.9 & 1050 & 10.9 & $1.1 \times 10^{9}$ \\
\hline 3 & A2 & $\mid r-I$ & 101.1 & 3550 & 11.3 & $9.3 \times 10^{8}$ \\
\hline 4 & A2 & Ir-2 & 101.1 & 3550 & 13.8 & $6.8 \times 10^{7}$ \\
\hline 5 & A3 & $\mid r-I$ & 101.6 & 1390 & 11.2 & $9.3 \times 10^{8}$ \\
\hline 6 & A3 & Ir-2 & 101.6 & 1390 & 13.7 & $7.5 \times 10^{7}$ \\
\hline 7 & A4 & $|r-|$ & 100.0 & 1500 & 9.7 & $3.0 \times 10^{9}$ \\
\hline 8 & A4 & Ir-2 & 100.0 & 1500 & 12.2 & $1.8 \times 10^{8}$ \\
\hline 9 & TI & $\mid r-I$ & 79.1 & 200 & -12.4 & $9.5 \times 10^{9}$ \\
\hline 10 & TI & Ir-2 & 79.1 & 200 & -9.8 & $3.6 \times 10^{9}$ \\
\hline 11 & $\mathrm{~T} 2$ & $|r-|$ & 76.9 & 44 & -15.6 & $1.0 \times 10^{10}$ \\
\hline 12 & $\mathrm{~T} 2$ & Ir-2 & 76.9 & 44 & -13.0 & $7.0 \times 10^{9}$ \\
\hline 13 & T3 & $|r-|$ & 84.0 & 5600 & -5.5 & $4.0 \times 10^{9}$ \\
\hline 14 & T3 & Ir-2 & 84.0 & 5600 & -3.0 & $1.0 \times 10^{9}$ \\
\hline 15 & $\mathrm{~T} 4$ & $|r-|$ & 81.3 & 2150 & -8.8 & $8.3 \times 10^{9}$ \\
\hline 16 & T4 & Ir-2 & 81.3 & 2150 & -6.3 & $2.2 \times 10^{9}$ \\
\hline
\end{tabular}

We employed the same luminescence experimental method formulated in our previous study. ${ }^{1}$ Details of the method are discussed in both the main text and the supporting information. Pertaining to the luminescence, the counterpart of "ketone" in the previous study are "thiol" or "amide" in this study; the counterpart of "acid" in our previous study is "base" in this study; the counterpart of "reductant" is "oxidant" in this study. Therefore, in this study $S_{0}$ is the initial concentration of thiol or amide in the solution prepared, and $A_{0}$ is the initial concentration of base in the solution prepared.

In this study, in all entries In table S2 except entries 11 and 12, amide or thiol does not quench the whereas the base dynamically quench the luminescence intensity of Ir oxidants. In entries 11 and 12, both thiol and base dynamically quench the luminescence intensity of the Ir oxidants.

In our previous study, we mentioned the following: ${ }^{1}$

"More rigorously, it is the unbound ketone and/or acid that result in the non-PCET quenching. Thus, pertaining to non-PCET quenching, the $\mathrm{H}$-bonded adduct concentration should be subtracted from the initial concentration of the non-PCET quencher. For example, if the ketone does not quench and the acid dynamically quench the luminescence intensity, equation 4 in the main text is modified to equation S6a and rearranged to equation S6b. 


$$
\begin{aligned}
& \frac{\mathrm{I}_{0}}{\mathrm{I}}=1+\mathrm{k}_{\mathrm{SV}}^{\prime}\left(\mathrm{A}_{0}-\mathrm{x}\right)+\mathrm{k}_{\mathrm{PCET}} \tau_{0}(\mathrm{x}) \\
& \frac{\mathrm{I}_{0}}{\mathrm{I}}=1+\mathrm{k}^{\prime}{ }_{\mathrm{SV}} \mathrm{A}_{0}+\left(\mathrm{k}_{\mathrm{PCET}} \tau_{0}-\mathrm{k}^{\prime}{ }_{\mathrm{SV}}\right)(\mathrm{x})
\end{aligned}
$$

Therefore, "k $\mathrm{kCET}_{\mathrm{PCT}} \tau_{0}$ " determined is actually $\left(k_{\mathrm{PCET}} \tau_{0}-k^{\prime} \mathrm{sv}\right)$.

As will be shown in Section 5 below, the non-PCET dynamic quenching constants are negligible (typically less than $2 \%)$ compared to the PCET rate constants, i.e. $\left(k_{\mathrm{PCET}} \tau_{0}-k_{\text {SV }}^{\prime}\right) \gg k^{\prime}$ sv. Thus, we nevertheless treat the non-PCET quenching according to equations S2-S5 described above for simplicity, and report ( $\left.k_{\mathrm{PCET}} \tau_{0}-k^{\prime}{ }_{\mathrm{SV}}\right)$ as $k_{\mathrm{PCET}} \tau_{0}$. i.e. Equation $\mathrm{S} 7$ is used instead of equations S6b."

$$
\frac{\mathrm{I}_{0}}{\mathrm{I}}=1+k^{\prime}{ }_{\mathrm{SV}} \mathrm{A}_{0}+k_{\mathrm{PCET}} \tau_{0}(\mathrm{x})
$$

In this study, however, the non-PCET quenching by the base alone is no longer negligible relative to the PCET rate constants of certain combinations of amide and oxidant. Therefore, equation S6b is employed. We define a new term, $k_{\text {PCET }}^{\prime} \tau_{0}$ in which $k_{\text {PCET }}^{\prime} \tau_{0}=\left(k_{\text {PCET }} \tau_{0}-k_{S V}^{\prime}\right)$ and $k_{S V}^{\prime}$ is the dynamic quenching constant by the base alone. The key equation is modified to equation (a)

$$
S_{0}+A_{0}=\frac{S_{0} A_{0} k_{o b s} \tau_{0}}{\boldsymbol{k}_{\text {PCET }}^{\prime} \tau_{0}}+\frac{\boldsymbol{k}_{\text {PCET }}^{\prime} \tau_{0}}{k_{\text {obs }} \tau_{0}}-\frac{1}{K_{A}}
$$

$k_{\text {PCET }}^{\prime} \tau_{0}$ are $K_{\mathrm{A}}$ are found then. $k_{\mathrm{PCET}} \tau_{0}$ is obtained by equation (b)

$$
k_{\text {PCET }} \tau_{0}=k_{\text {PCET }}^{\prime} \tau_{0}+k^{\prime}{ }_{S V}(\mathrm{~b})
$$

In our previous study, we mentioned the following right after: ${ }^{1}$

"In cases where the ketone dynamically quenches the luminescence intensity, our chosen values of $S_{0}$ are all much higher than that of $A_{0}$ thus even more higher than the adduct concentration $x$, making $\left(S_{0}-x\right) \approx S_{0}$. Thus, this concern is not relevant."

Similarly, in this study Table S2 entries 11 and 12, our chosen values of $S_{0}$ (initial concentration of thiol T2) are much higher than the adduct concentration $\mathrm{x}$, making $\left(\mathrm{S}_{0}-\mathrm{x}\right) \approx \mathrm{S}_{0}$. Thus, this concern is also not relevant here. 


\subsection{Entry 1.}

Amide does not quench the luminescence of oxidant *Ir (III). Non-PCET quenching of oxidant by the base alone (without amide or thiol) is given by the following equation:

$$
\mathrm{I}_{0} / \mathrm{I}=1+k_{\mathrm{SV}}^{\prime} \mathrm{A}_{0}
$$

In all experiments, the solution was irradiated at $380 \mathrm{~nm}$, and fluorescence was measured at $496 \mathrm{~nm}$.

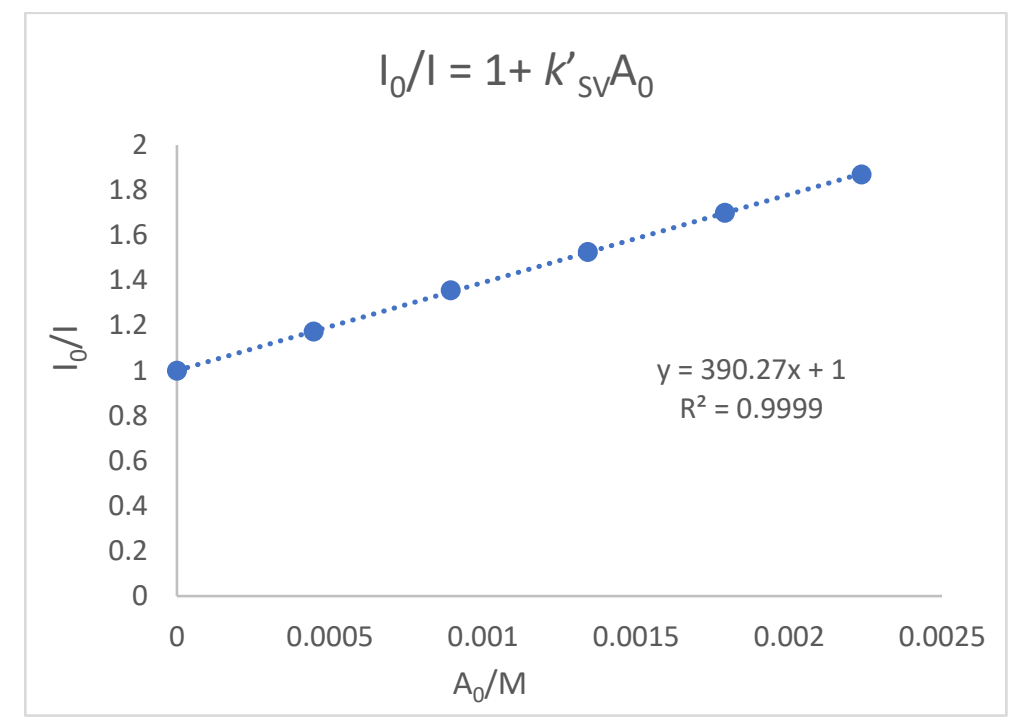

Figure S2.1. Stern-Volmer plot of oxidant *Ir (III) luminescence data of non-PCET quenching by base alone

$$
k^{\prime}{ }_{S V}=390
$$

Time-resolved luminescence suggests that the non-PCET quenching by base is dynamic, therefore, the following equation is used:

$$
\frac{I_{0}}{I}=1+\left(k^{\prime}{ }_{S V}+k_{o b s} \tau_{0} S_{0}\right) A_{0}
$$


Table. S2.1.1. $\mathrm{S}_{0}$ fixed at $0.0025 \mathrm{M}, \mathrm{A}_{0}$ varied from 0.000075 to $0.000175 \mathrm{M}$

\begin{tabular}{ccccccc}
\hline $\mathbf{A}_{0} / \mathbf{M}$ & $\mathbf{S}_{0} \mathbf{A}_{0} / \mathbf{M}^{2}$ & I/au 1 & I/au 2 & I/au 3 & I/au ave & $I_{0} / \mathbf{I}$ \\
0 & 0 & & & & & 1 \\
0.000075 & $1.875 \mathrm{E}-07$ & 167 & 166 & 166 & 166.3333 & 1.839679 \\
0.0001 & 0.00000025 & 145 & 145 & 145 & 145 & 2.110345 \\
0.000125 & $3.125 \mathrm{E}-07$ & 128 & 129 & 127 & 128 & 2.390625 \\
0.00015 & 0.000000375 & 116 & 116 & 116 & 116 & 2.637931 \\
0.000175 & $4.375 \mathrm{E}-07$ & 106 & 106 & 106 & 106 & 2.886792 \\
\hline
\end{tabular}

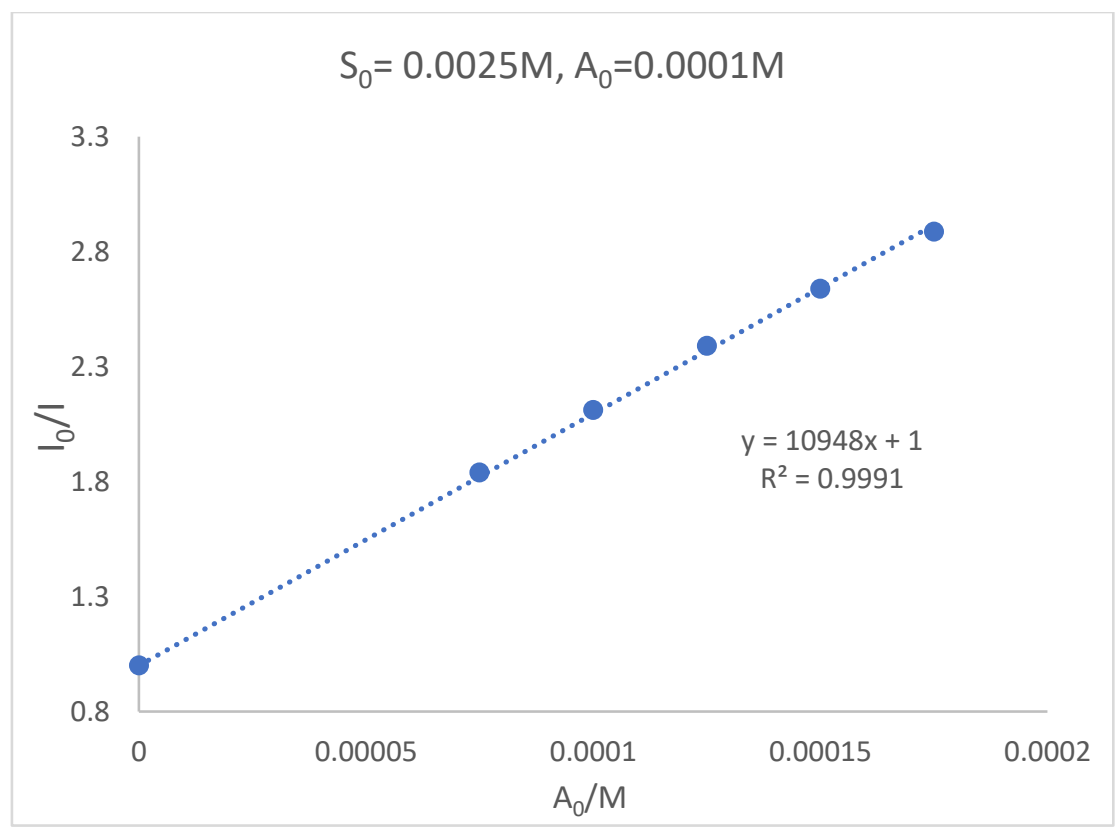

Figure S2.1.1. Stern-Volmer plot of luminescence data in Table S2.1.1.

$I_{0}$ was extrapolated from the five data points.

$$
\begin{gathered}
k_{S V}^{\prime}+k_{o b s} \tau_{0} S_{0}=10948 \\
390+k_{o b s} \tau_{0} \times 0.0025=10948, k_{o b s} \tau_{0}=4223200
\end{gathered}
$$


Table. S2.1.2. $S_{0}$ fixed at $0.0005 \mathrm{M}, \mathrm{A}_{0}$ varied from 0.00015 to $0.00025 \mathrm{M}$

\begin{tabular}{ccccccc}
\hline $\mathbf{A}_{0} / \mathbf{M}$ & $\mathbf{S}_{0} \mathbf{A}_{0} / \mathbf{M}^{2}$ & I/au 1 & I/au 2 & I/au 3 & I/au ave & $I_{0} / \mathbf{I}$ \\
0 & 0 & & & & & 1 \\
0.00015 & 0.000000075 & 189 & 190 & 191 & 190 & 1.773684 \\
0.000175 & $8.75 \mathrm{E}-08$ & 178 & 177 & 179 & 178 & 1.893258 \\
0.0002 & 0.0000001 & 167 & 166 & 168 & 167 & 2.017964 \\
0.000225 & $1.125 \mathrm{E}-07$ & 160 & 160 & 160 & 160 & 2.10625 \\
0.00025 & 0.000000125 & 150 & 151 & 152 & 151 & 2.231788 \\
\hline
\end{tabular}

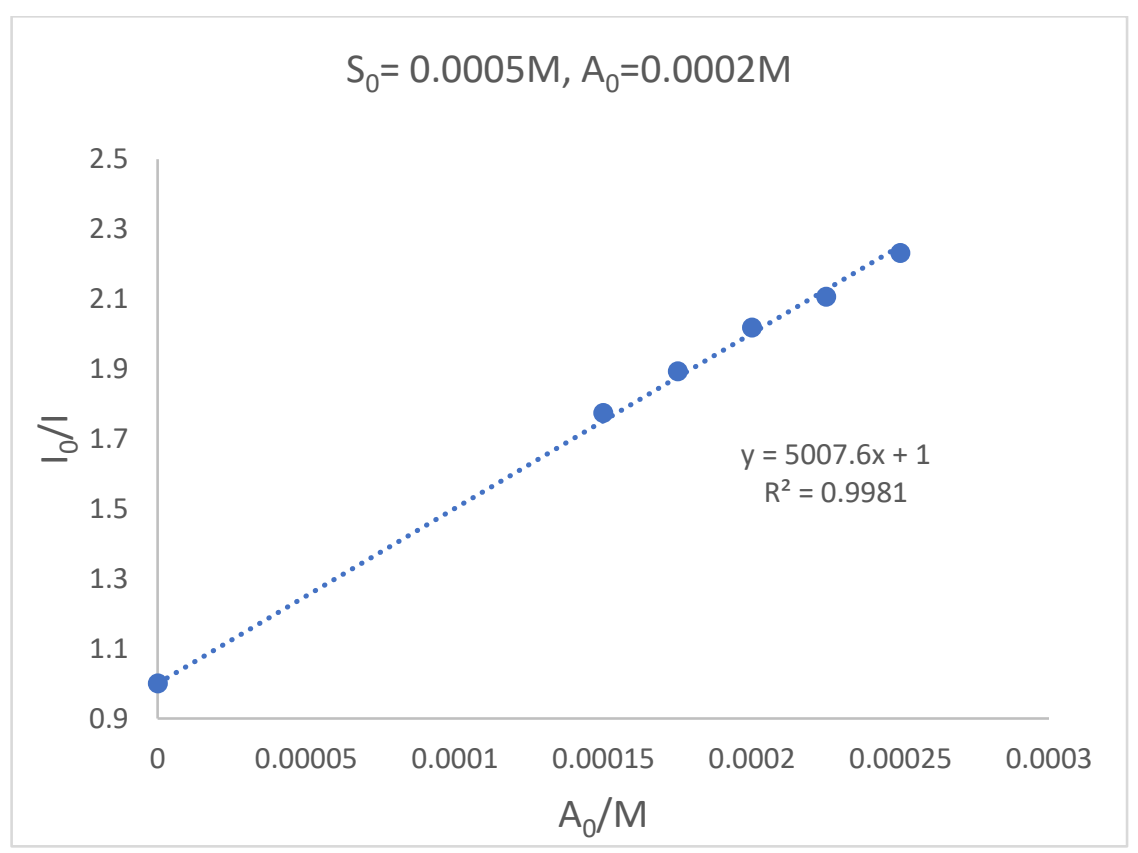

Figure S2.1.2. Stern-Volmer plot of luminescence data in Table S2.1.2.

$$
\begin{aligned}
& I_{0} \text { was extrapolated from the five data points. } \\
& \qquad k^{\prime}{ }_{S V}+k_{o b s} \tau_{0} S_{0}=5008 \\
& 390+k_{o b s} \tau_{0} \times 0.0005=50085, k_{o b s} \tau_{0}=9236000
\end{aligned}
$$


Table. S2.1.3. $S_{0}$ fixed at $0.005 \mathrm{M}, \mathrm{A}_{0}$ varied from 0.000075 to $0.00015 \mathrm{M}$

\begin{tabular}{ccccccc}
\hline $\mathbf{A}_{0} / \mathbf{M}$ & $\mathbf{S}_{0} \mathbf{A}_{0} / \mathbf{M}^{2}$ & I/au 1 & I/au 2 & I/au 3 & I/au ave & $I_{0} / \mathbf{I}$ \\
0 & 0 & & & & & 1 \\
0.00005 & 0.00000025 & 189 & 188 & 189 & 188.6667 & 1.590106 \\
0.000075 & $3.75 \mathrm{E}-07$ & 153 & 151 & 151 & 151.6667 & 1.978022 \\
0.0001 & 0.0000005 & 129 & 129 & 130 & 129.3333 & 2.319588 \\
0.000125 & $6.25 \mathrm{E}-07$ & 116 & 115 & 115 & 115.3333 & 2.601156 \\
0.00015 & 0.00000075 & 103 & 103 & 103 & 103 & 2.912621 \\
\hline
\end{tabular}

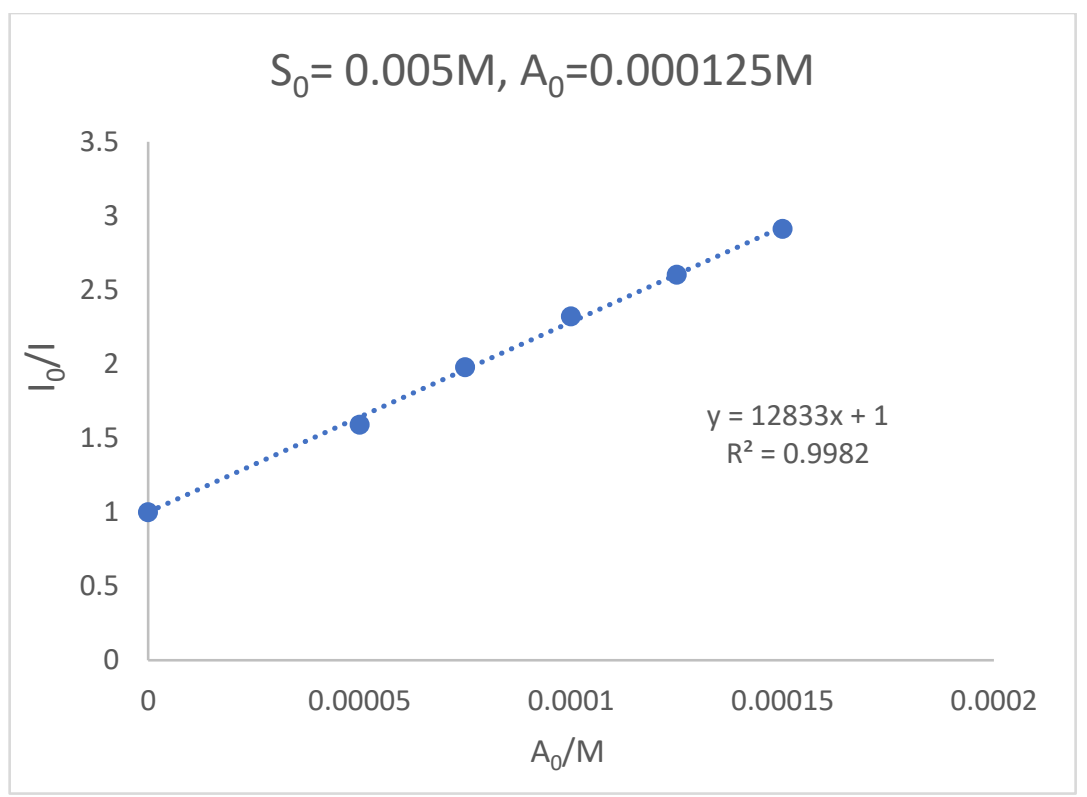

Figure S2.1.3. Stern-Volmer plot of luminescence data in Table S2.1.3.

$I_{0}$ was extrapolated from the four data points.

$$
\begin{gathered}
k^{\prime}{ }_{S V}+k_{o b s} \tau_{0} S_{0}=12833 \\
390+k_{o b s} \tau_{0} \times 0.005=12833, k_{o b s} \tau_{0}=2488600
\end{gathered}
$$


Table. S2.1.4. $S_{0}$ fixed at $0.001 \mathrm{M}, \mathrm{A}_{0}$ varied from 0.0001 to $0.000175 \mathrm{M}$

\begin{tabular}{ccccccc}
\hline $\mathbf{A}_{0} / \mathrm{M}$ & $\mathrm{S}_{0} \mathbf{A}_{0} / \mathrm{M}^{2}$ & I/au 1 & I/au 2 & I/au 3 & I/au ave & $\mathrm{I}_{0} / \mathbf{I}$ \\
0 & 0 & & & & & 1 \\
0.0001 & 0.0000001 & 176 & 177 & 176 & 176.3333 & 1.741021 \\
0.000125 & 0.000000125 & 159 & 159 & 158 & 158.6667 & 1.934874 \\
0.00015 & 0.00000015 & 146 & 146 & 146 & 146 & 2.10274 \\
0.000175 & 0.000000175 & 136 & 136 & 136 & 136 & 2.257353 \\
\hline
\end{tabular}

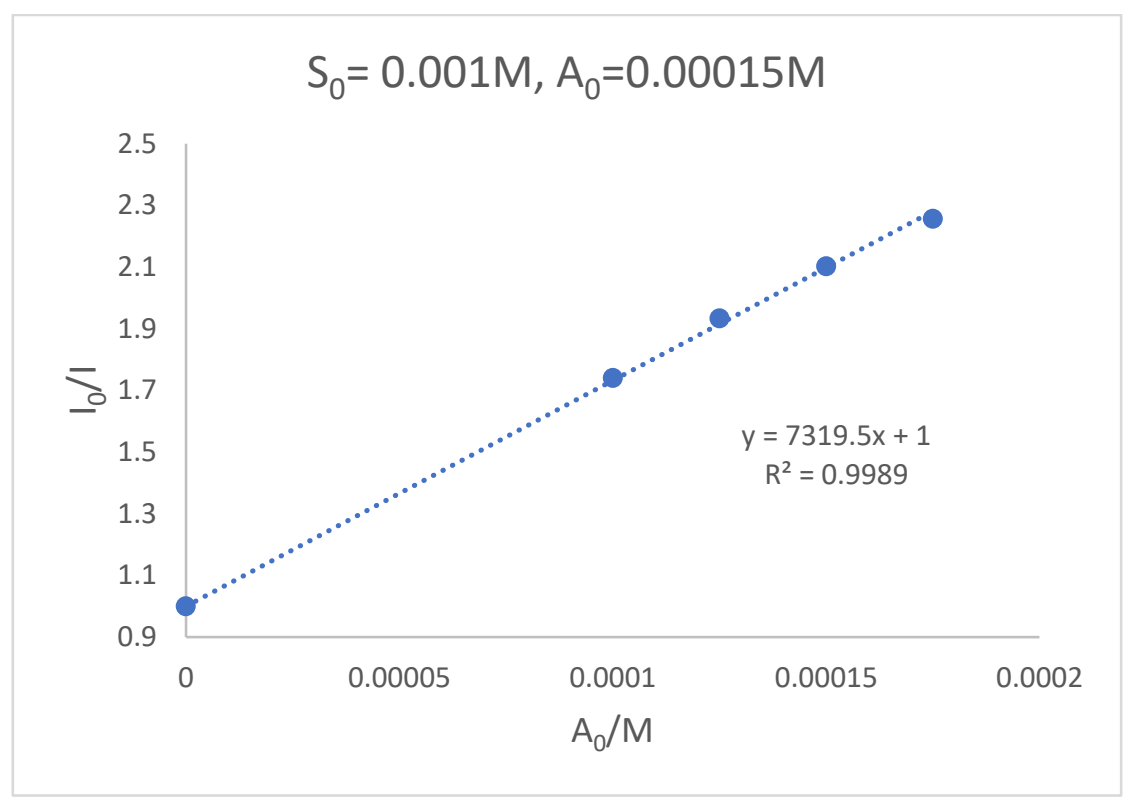

Figure S2.1.4. Stern-Volmer plot of luminescence data in Table S2.1.4.

$$
\begin{aligned}
& I_{0} \text { was extrapolated from the four data points. } \\
& \qquad k^{\prime}{ }_{S V}+k_{o b s} \tau_{0} S_{0}=7320 \\
& 390+k_{o b s} \tau_{0} \times 0.005=7320, k_{o b s} \tau_{0}=6930000
\end{aligned}
$$


Table 2.1.5. Solutions to Key Equation a and Entry 1 in Table S2.

\begin{tabular}{|c|c|c|c|c|c|}
\hline & $S_{0}(M)$ & $A_{0}(M)$ & $S_{0} A_{0}\left(M^{2}\right)$ & $S_{0}+A_{0}(M)$ & $k_{o b s} \tau_{0}$ \\
\hline 1 & 0.0025 & 0.000125 & $3.125 \mathrm{E}-07$ & 0.002625 & 4223200 \\
\hline 2 & 0.0005 & 0.0002 & 0.0000001 & 0.0007 & 9236000 \\
\hline 3 & 0.005 & 0.0001 & 0.0000005 & 0.0051 & 2488600 \\
\hline \multirow[t]{14}{*}{4} & 0.001 & 0.000125 & $1.25 \mathrm{E}-07$ & 0.001125 & 6930000 \\
\hline & & & $K_{\mathrm{A}} / \mathrm{M}^{-1}$ & $k_{\text {PCET }}^{\prime} \tau_{0} / M^{-1}$ & \\
\hline & & $1 \& 2$ & 1040 & 14770 & \\
\hline & & $1 \& 3$ & 980 & 15030 & \\
\hline & & $1 \& 4$ & 820 & 15910 & \\
\hline & & $2 \& 3$ & 1020 & 14920 & \\
\hline & & $2 \& 4$ & 1500 & 11930 & \\
\hline & & $3 \& 4$ & 880 & 15340 & \\
\hline & & average & 1050 & 14650 & \\
\hline & \multicolumn{4}{|c|}{$k_{\mathrm{PCET}} \tau_{0}=k_{\mathrm{PCET}}^{\prime} \tau_{0}+k_{\mathrm{SV}}^{\prime}=14650+390=15040$} & \\
\hline & \multicolumn{4}{|c|}{$\Delta G^{\circ}{ }_{\mathrm{H} \text {-bond }}(\mathrm{kcal} / \mathrm{mol})=-\mathrm{R} T \ln K_{\mathrm{A}}=-4.12$} & \\
\hline & \multicolumn{4}{|c|}{$\ln \left[k_{\text {PCET }}\left(\mathrm{M}^{-1} \mathrm{~S}^{-1}\right)\right]=\ln \left[k_{\text {PCET }} \mathrm{T}_{0} / \tau_{0}\right]=22.86$} & \\
\hline & \multicolumn{4}{|c|}{$\tau_{0}=1.8 \mu \mathrm{s}$} & \\
\hline & \multicolumn{4}{|c|}{ error in $\Delta G^{\circ}{ }_{\text {-bond }}(\mathrm{kcal} / \mathrm{mol})=0.15$} & \\
\hline
\end{tabular}




\subsection{Entry 2 .}

Amide does not quench the luminescence of oxidant *Ir (III). Non-PCET quenching of oxidant by the base alone (without amide or thiol) is given by the following equation:

$$
\mathrm{I} / \mathrm{I}=1+k_{\mathrm{sv}}^{\prime} A_{0}
$$

In all experiments, the solution was irradiated at $370 \mathrm{~nm}$, and fluorescence was measured at $470 \mathrm{~nm}$.

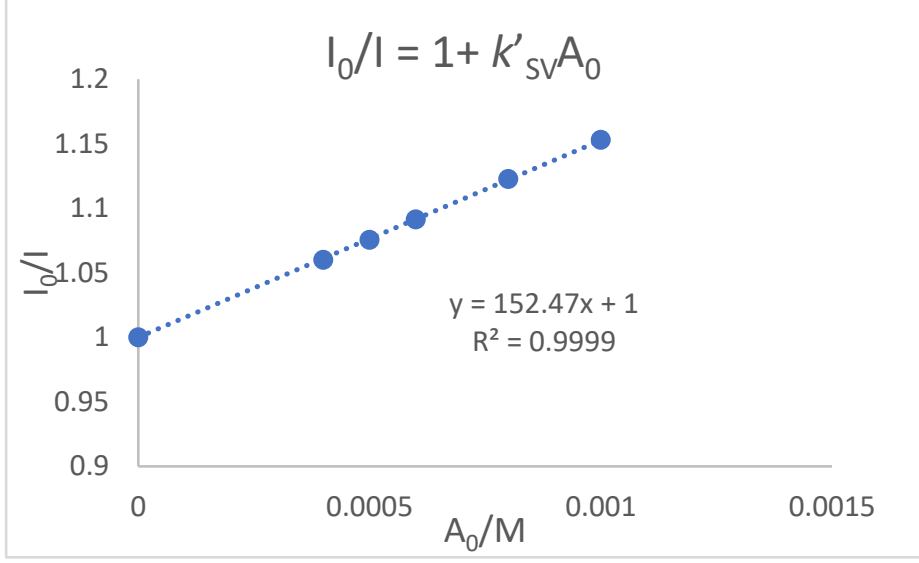

Figure S2.2.a Stern-Volmer plot of oxidant luminescence data of non-PCET quenching by base alone

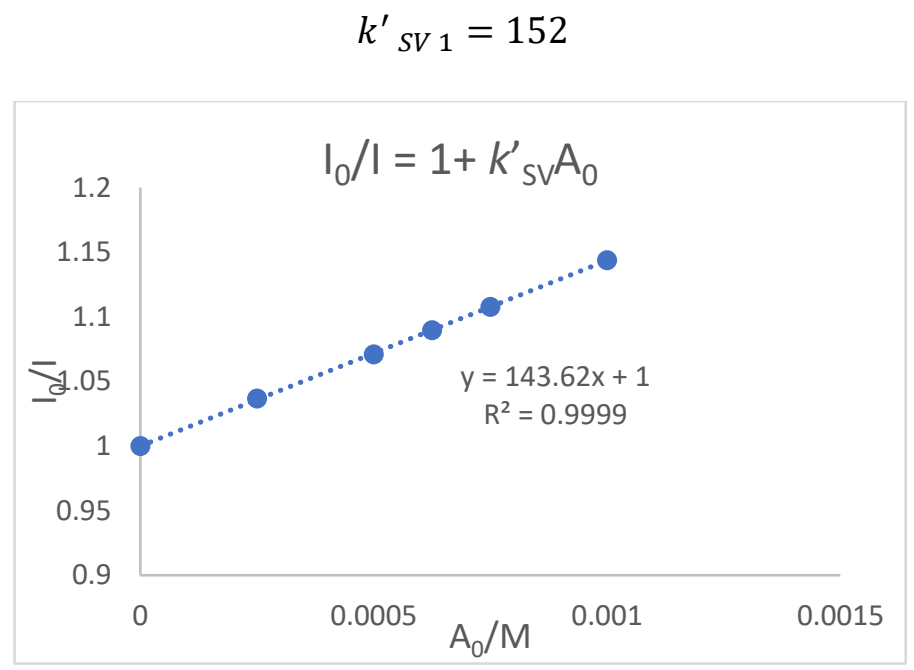

Figure S2.2.b Repeat Stern-Volmer plot of oxidant luminescence data of non-PCET quenching by base alone

$$
\begin{gathered}
k_{S V 2}^{\prime}=144 \\
k_{S V}^{\prime}=148 \pm 4
\end{gathered}
$$


Time-resolved luminescence suggests that the non-PCET quenching by base is dynamic, therefore, the following equation is used:

$$
\frac{I_{0}}{I}=1+\left(k_{S V}^{\prime}+k_{o b s} \tau_{0} S_{0}\right) A_{0}
$$

Table S2.2.1. Luminescence quenching data with $S_{0}=0.0025 \mathrm{M}, A_{0}=0.0001 \mathrm{M}$. $S_{0}$ fixed at $0.0025 \mathrm{M}$. $A_{0}$ varied from 0.00005 to $0.00015 \mathrm{M}$.

\begin{tabular}{ccccccc}
\hline $\mathbf{A}_{0} / \mathbf{M}$ & $\mathbf{S}_{0} \mathbf{A}_{0} / \mathbf{M}^{2}$ & I/au 1 & I/au 2 & I/au 3 & I/au ave & $I_{0} / \mathbf{I}$ \\
0 & 0 & & & & & 1 \\
0.00005 & $1.25 \mathrm{E}-07$ & 630 & 630 & 631 & 630.3333 & 1.099418 \\
0.000075 & $1.88 \mathrm{E}-07$ & 603 & 603 & 603 & 603 & 1.149254 \\
0.0001 & $2.5 \mathrm{E}-07$ & 575 & 579 & 579 & 577.6667 & 1.199654 \\
0.000125 & $3.13 \mathrm{E}-07$ & 555 & 555 & 555 & 555 & 1.248649 \\
0.00015 & $3.75 \mathrm{E}-07$ & 534 & 535 & 535 & 534.6667 & 1.296135 \\
\hline
\end{tabular}

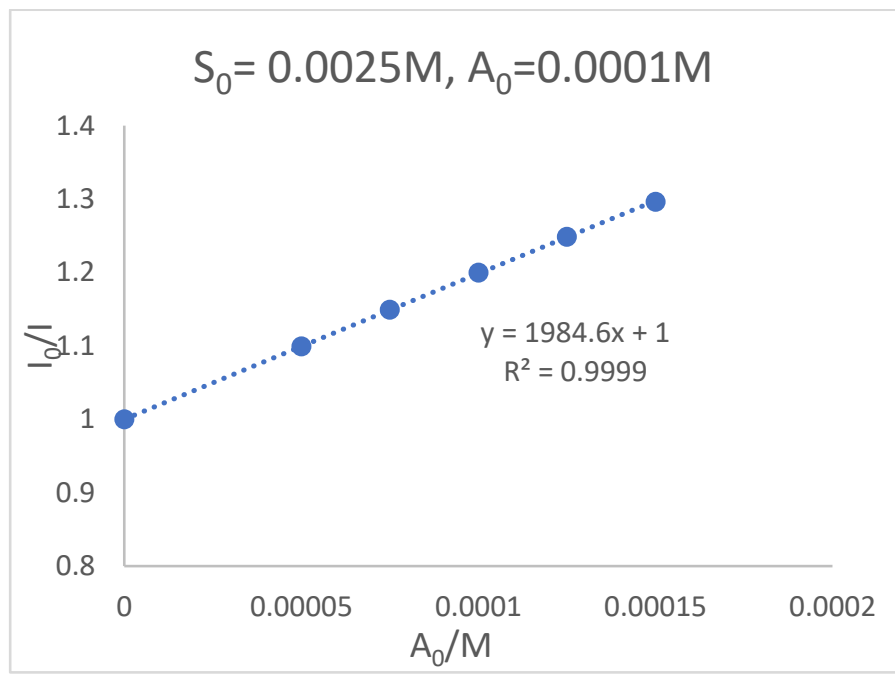

Figure S2.2.1. Stern-Volmer plot of luminescence data in Table S2.2.1.

$$
\begin{aligned}
& I_{0} \text { was extrapolated from the five data points. } \\
& \qquad k^{\prime}{ }_{S V}+k_{o b s} \tau_{0} S_{0}=1985 \\
& 148+k_{o b s} \tau_{0} \times 0.0025=1985, k_{o b s} \tau_{0}=734800
\end{aligned}
$$


Table S2.2.2. Repeat. Luminescence quenching data with $S_{0}=0.0025 \mathrm{M}, A_{0}=0.0001 \mathrm{M}$. $S_{0}$ fixed at $0.0025 \mathrm{M}$. $A_{0}$ varied from 0.00005 to $0.00015 \mathrm{M}$.

\begin{tabular}{ccccccc}
\hline$A_{0} / \mathbf{M}$ & $\mathbf{S}_{0} A_{0} / M^{2}$ & I/au 1 & I/au 2 & I/au 3 & I/au ave & $I_{0} / \mathbf{I}$ \\
0 & 0 & & & & & 1 \\
0.00005 & $2.5 \mathrm{E}-07$ & 810 & 810 & 810 & 810 & 1.085185 \\
0.000075 & $3.75 \mathrm{E}-07$ & 780 & 780 & 781 & 780.3333 & 1.126442 \\
0.0001 & $5 \mathrm{E}-07$ & 754 & 752 & 753 & 753 & 1.167331 \\
0.000125 & $6.25 \mathrm{E}-07$ & 728 & 729 & 729 & 728.6667 & 1.206313 \\
0.00015 & $7.5 \mathrm{E}-07$ & 705 & 704 & 704 & 704.3333 & 1.247989 \\
\hline
\end{tabular}

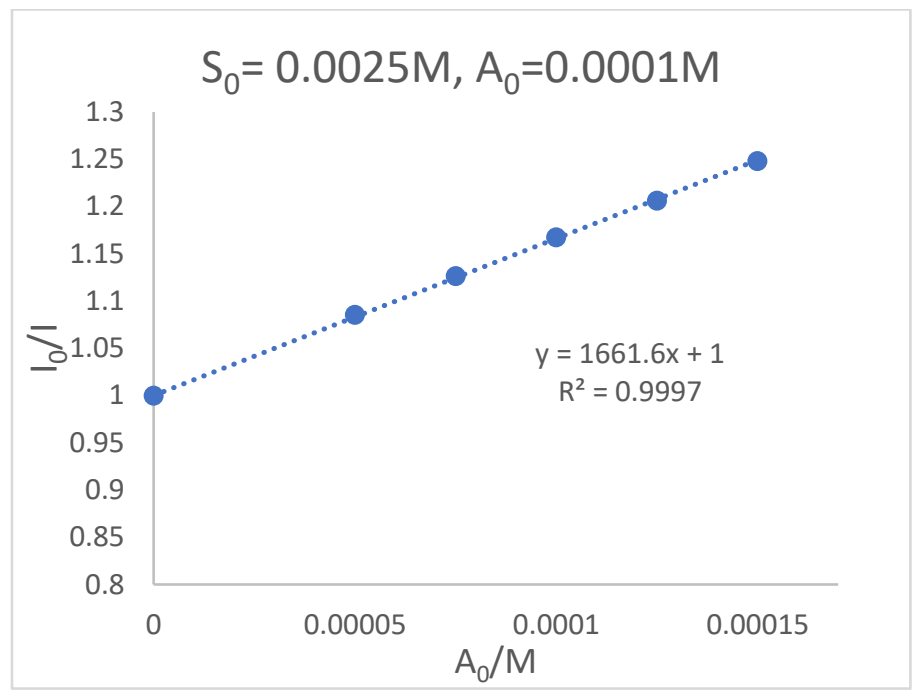

Figure S2.2.2. Stern-Volmer plot of luminescence data in Table S2.2.2.

$I_{0}$ was extrapolated from the five data points.

$$
\begin{gathered}
k^{\prime}{ }_{S V}+k_{\text {obs }} \tau_{0} S_{0}=1662 \\
148+k_{\text {obs }} \tau_{0} \times 0.0025=1662, k_{\text {obs }} \tau_{0}=605600 \\
k_{\text {obs average }} \tau_{0}=670200 \pm 64600
\end{gathered}
$$

Substitute $K_{\mathrm{A}}=1045$ (from entry 1), $k_{o b s} \tau_{0}=670200$ into the equation

$$
\begin{gathered}
S_{0}+A_{0}=\frac{S_{0} A_{0} k_{\text {obs }} \tau_{0}}{k_{P C E T} \tau_{0}}+\frac{k_{P C E T} \tau_{0}}{k_{o b s} \tau_{0}}-\frac{1}{K_{A}} \\
0.0025+0.0001=\frac{0.0025 \times 0.0001 \times 670200}{k_{P C E T} \tau_{0}}+\frac{k_{P C E T} \tau_{0}}{670200}-\frac{1}{1050}
\end{gathered}
$$




$$
\begin{gathered}
k_{\text {PCET }}^{\prime} \tau_{0}=2380 \\
k_{\text {PCET }} \tau_{0}=k_{\text {PCET }}^{\prime} \tau_{0}+k_{\text {SV }}^{\prime}=2380+148=2530 \\
\Delta G^{\circ}{ }_{\text {-bond }}(\mathrm{kcal} / \mathrm{mol})=-\mathrm{RT} \ln K_{\mathrm{A}}=-4.12 \\
\ln \left[k_{\mathrm{PCET}}\left(\mathrm{M}^{-1} \mathrm{~s}^{-1}\right)\right]=\ln \left[k_{\mathrm{PCET}} \tau_{0} / \tau_{0}\right]=20.83 \\
\tau_{0}=2.3 \mu \mathrm{S} \\
\text { error in } \Delta G^{\circ}{ }_{\mathrm{H} \text {-bond }}(\mathrm{kcal} / \mathrm{mol})=0.12 \\
\text { error in } \ln \left[k_{\mathrm{PCET}}\left(\mathrm{M}^{-1} \mathrm{~s}^{-1}\right)\right]=0.12
\end{gathered}
$$

\subsection{Entry 3.}

Amide does not quench the luminescence of oxidant *Ir (III), therefore, the following equation is used:

$$
\frac{I_{0}}{I}=1+\left(k^{\prime}{ }_{S V}+k_{o b s} \tau_{0} S_{0}\right) A_{0}
$$

Table. S2.3.1. $S_{0}$ fixed at $0.006 \mathrm{M}, \mathrm{A}_{0}$ varied from 0.000025 to $0.00015 \mathrm{M}$

\begin{tabular}{ccccccc}
\hline$A_{0} / \mathbf{M}$ & $\mathbf{S}_{0} \mathbf{A}_{0} / \mathbf{M}^{2}$ & I/au 1 & I/au 2 & I/au 3 & I/au ave & $I_{0} / \mathbf{I}$ \\
0 & 0 & & & & & 1 \\
0.000025 & 0.00000015 & 498 & 492 & 496 & 495.3333 & 1.040027 \\
0.00005 & 0.0000003 & 480 & 482 & 480 & 480.6667 & 1.071761 \\
0.0001 & 0.0000006 & 442 & 443 & 445 & 443.3333 & 1.162015 \\
0.000125 & 0.00000075 & 429 & 428 & 427 & 428 & 1.203645 \\
0.00015 & 0.0000009 & 418 & 415 & 415 & 416 & 1.238365 \\
\hline
\end{tabular}




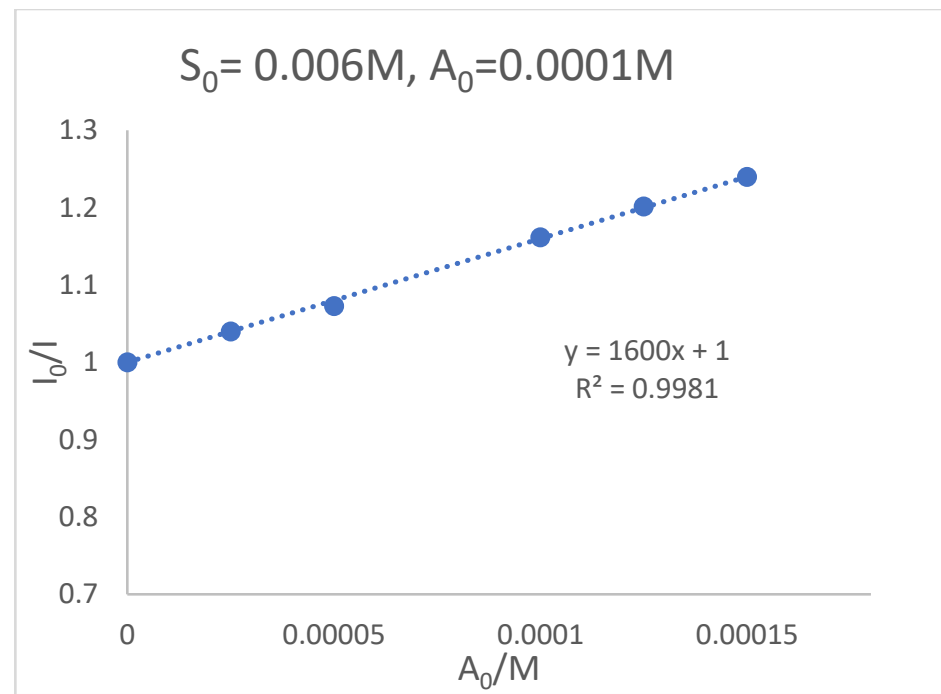

Figure S2.3.1. Stern-Volmer plot of luminescence data in Table S2.3.1.

$I_{0}$ was extrapolated from the five data points.

$$
\begin{gathered}
k^{\prime}{ }_{S V}+k_{o b s} \tau_{0} S_{0}=1600 \\
390+k_{o b s} \tau_{0} \times 0.006=1600, k_{o b s} \tau_{0}=201700
\end{gathered}
$$

Table. S2.3.2. $\mathrm{S}_{0}$ fixed at $0.003 \mathrm{M}, \mathrm{A}_{0}$ varied from 0.00015 to $0.0003 \mathrm{M}$

\begin{tabular}{ccccccc}
\hline $\mathbf{A}_{0} / \mathbf{M}$ & $\mathbf{S}_{0} \mathbf{A}_{0} / \mathbf{M}^{2}$ & I/au 1 & I/au 2 & I/au 3 & I/au ave & $I_{0} / \mathbf{I}$ \\
0 & 0 & & & & & 1 \\
0.00015 & 0.00000045 & 349 & 347 & 347 & 347.6667 & 1.239406 \\
0.0002 & 0.0000006 & 332 & 331 & 331 & 331.3333 & 1.300503 \\
0.00025 & 0.00000075 & 310 & 310 & 310 & 310 & 1.39 \\
0.0003 & 0.0000009 & 296 & 294 & 294 & 294.6667 & 1.46233 \\
\hline
\end{tabular}




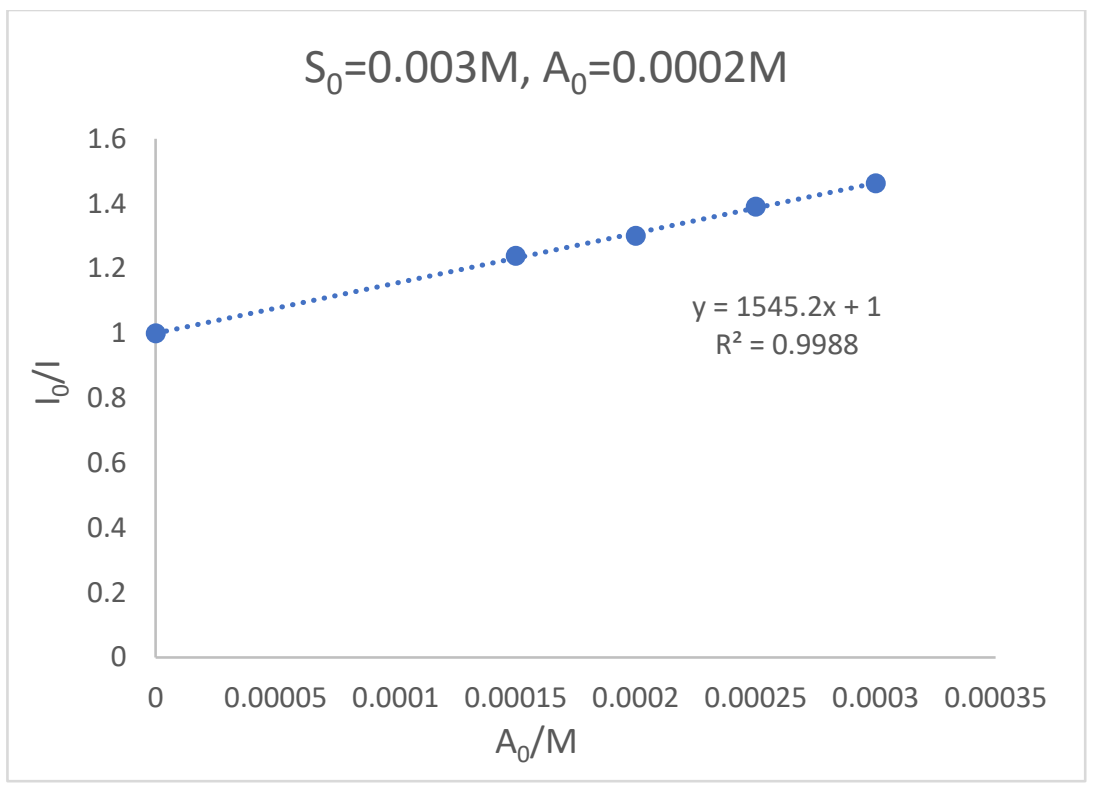

Figure S2.3.2. Stern-Volmer plot of luminescence data in Table S2.3.2.

$I_{0}$ was extrapolated from the four data points.

$$
\begin{gathered}
k^{\prime}{ }_{S V}+k_{o b s} \tau_{0} S_{0}=1545 \\
390+k_{o b s} \tau_{0} \times 0.003=1545, k_{o b s} \tau_{0}=385000
\end{gathered}
$$

Table. S2.3.3. $S_{0}$ fixed at $0.004 \mathrm{M}, \mathrm{A}_{0}$ varied from 0.00005 to $0.00025 \mathrm{M}$

\begin{tabular}{ccccccc}
\hline $\mathbf{A}_{0} / \mathbf{M}$ & $\mathbf{S}_{\mathbf{0}} \mathbf{A}_{0} / \mathbf{M}^{2}$ & I/au 1 & I/au 2 & I/au 3 & I/au ave & $I_{0} / \mathbf{I}$ \\
0 & 0 & & & & & 1 \\
0.00005 & 0.0000002 & 459 & 462 & 459 & 460 & 1.076087 \\
0.0001 & 0.0000004 & 427 & 428 & 427 & 427.3333 & 1.158346 \\
0.00015 & 0.0000006 & 401 & 399 & 400 & 400 & 1.2375 \\
0.0002 & 0.0000008 & 378 & 376 & 376 & 376.6667 & 1.314159 \\
0.00025 & 0.000001 & 356 & 354 & 354 & 354.6667 & 1.395677 \\
\hline
\end{tabular}




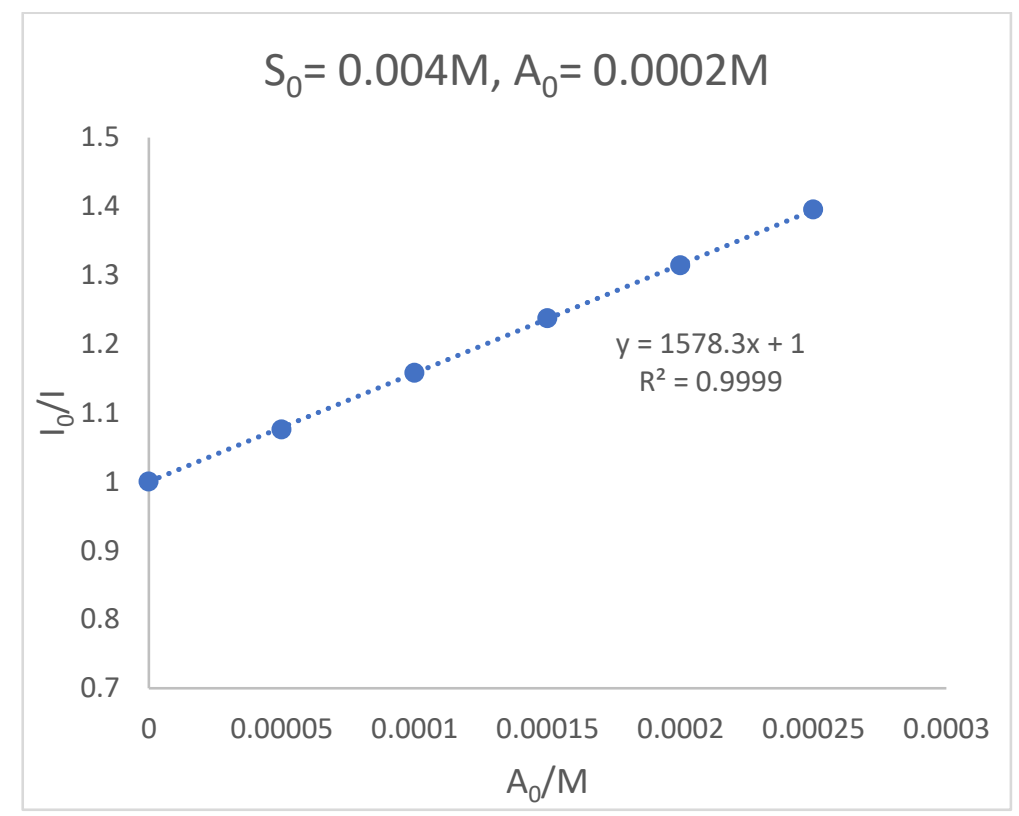

Figure S2.3.3. Stern-Volmer plot of luminescence data in Table S2.3.3.

$I_{0}$ was extrapolated from the five data points.

$$
\begin{gathered}
k^{\prime}{ }_{S V}+k_{o b s} \tau_{0} S_{0}=1578 \\
390+k_{o b s} \tau_{0} \times 0.003=1578, k_{o b s} \tau_{0}=297000
\end{gathered}
$$

Table. S2.3.4. $S_{0}$ fixed at $0.005 \mathrm{M}, \mathrm{A}_{0}$ varied from 0.000075 to $0.00015 \mathrm{M}$

\begin{tabular}{ccccccc}
\hline $\mathbf{A}_{0} / \mathbf{M}$ & $\mathbf{S}_{0} \mathbf{A}_{0} / \mathbf{M}^{2}$ & I/au 1 & I/au 2 & I/au 3 & I/au ave & $I_{0} / \mathbf{I}$ \\
0 & 0 & & & & & 1 \\
0.000075 & 0.00000045 & 456 & 457 & 457 & 456.6667 & 1.115036 \\
0.0001 & 0.0000006 & 437 & 439 & 439 & 438.3333 & 1.161673 \\
0.000125 & 0.00000075 & 426 & 426 & 426 & 426 & 1.195305 \\
0.00015 & 0.0000009 & 409 & 409 & 409 & 409 & 1.244988 \\
\hline
\end{tabular}


$S_{0}=0.005 \mathrm{M}, A_{0}=0.0001 M$

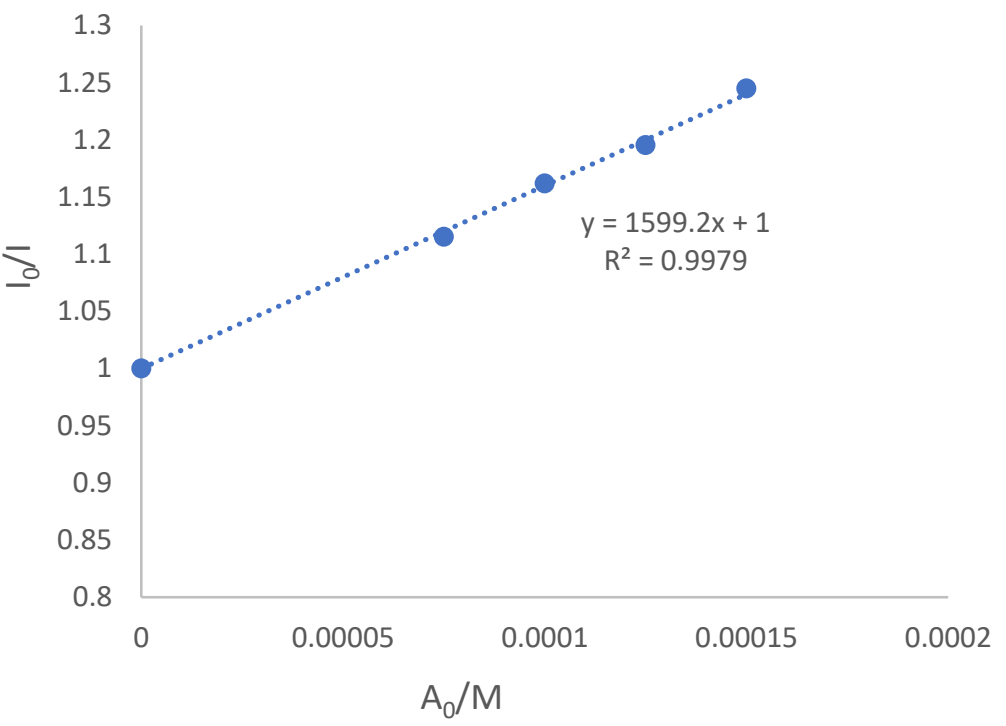

Figure S2.3.4. Stern-Volmer plot of luminescence data in Table S2.3.4.

$I_{0}$ was extrapolated from the four data points.

$$
\begin{gathered}
k^{\prime}{ }_{S V}+k_{o b s} \tau_{0} S_{0}=1599 \\
390+k_{o b s} \tau_{0} \times 0.005=1599, k_{o b s} \tau_{0}=241600
\end{gathered}
$$

Table. S2.3.5. $S_{0}$ fixed at $0.008 \mathrm{M}, A_{0}$ varied from 0.00008 to $0.0002 \mathrm{M}$

\begin{tabular}{ccccccc}
\hline $\mathbf{A}_{0} / \mathbf{M}$ & $\mathbf{S}_{0} \mathbf{A}_{0} / \mathbf{M}^{2}$ & I/au 1 & I/au 2 & I/au 3 & I/au ave & $I_{0} / \mathbf{I}$ \\
0 & 0 & & & & & 1 \\
0.00008 & $6.42 \mathrm{E}-07$ & 329 & 330 & 330 & 329.6667 & 1.130839 \\
0.000106667 & $8.57 \mathrm{E}-07$ & 316 & 318 & 317 & 317 & 1.176025 \\
0.000133333 & $1.07 \mathrm{E}-06$ & 306 & 306 & 307 & 306.3333 & 1.216975 \\
0.000213333 & $1.71 \mathrm{E}-06$ & 277 & 277 & 278 & 277.3333 & 1.344231 \\
\hline
\end{tabular}




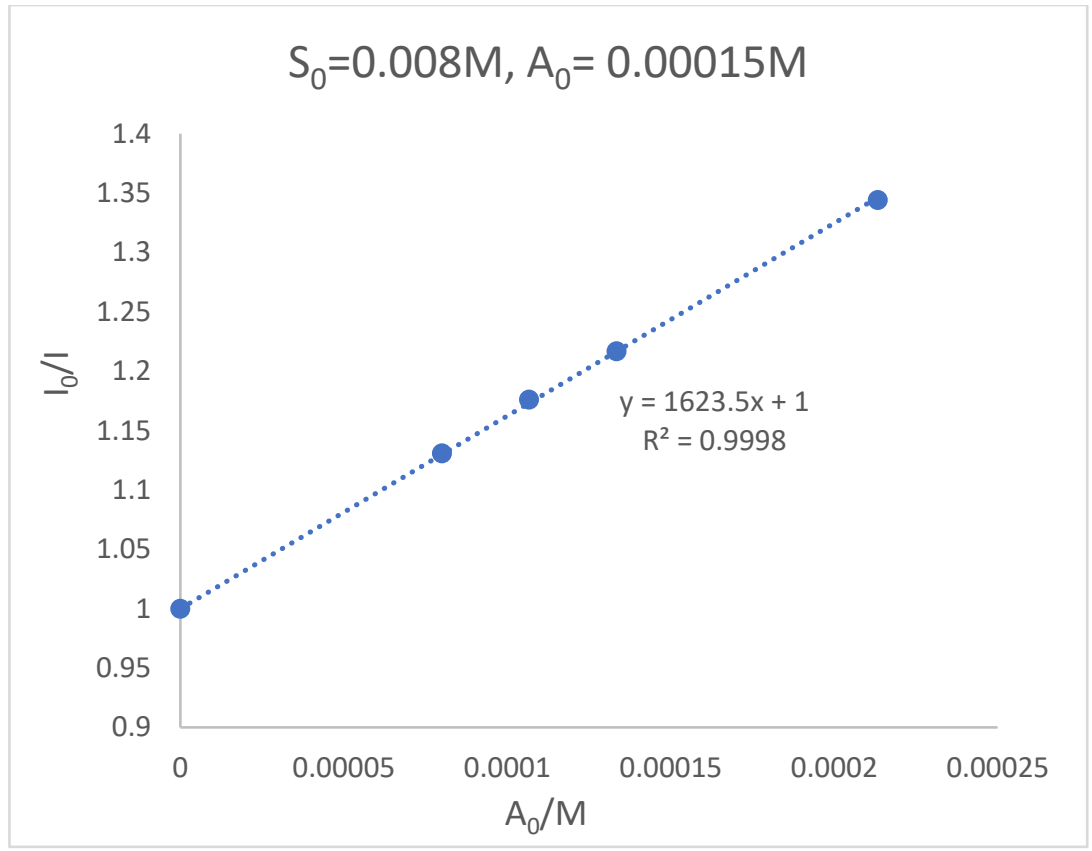

Figure S2.3.5. Stern-Volmer plot of luminescence data in Table S2.3.5.

$I_{0}$ was extrapolated from the four data points.

$$
\begin{gathered}
k^{\prime}{ }_{S V}+k_{o b s} \tau_{0} S_{0}=1623 \\
390+k_{o b s} \tau_{0} \times 0.005=1623, k_{o b s} \tau_{0}=153600
\end{gathered}
$$


Table 2.3.6. Solutions to Key Equation a and Entry 3 in Table S2.

\begin{tabular}{|c|c|c|c|c|c|}
\hline & $S_{0}(M)$ & $A_{0}(M)$ & $S_{0} A_{0}\left(M^{2}\right)$ & $S_{0}+A_{0}(M)$ & $k_{o b s} \tau_{0}$ \\
\hline 1 & 0.006 & 0.0001 & 0.0000006 & 0.0061 & 201700 \\
\hline 2 & 0.003 & 0.0002 & 0.0000006 & 0.0032 & 385000 \\
\hline 3 & 0.004 & 0.0002 & 0.0000008 & 0.0042 & 297000 \\
\hline 4 & 0.005 & 0.00011 & 0.00000055 & 0.00511 & 241600 \\
\hline \multirow[t]{18}{*}{5} & 0.008 & 0.00015 & 0.0000012 & 0.00815 & 153600 \\
\hline & & & $K_{\mathrm{A}} / \mathrm{M}^{-1}$ & $k_{\text {РСETTO}}^{\prime} / M^{-1}$ & \\
\hline & & $\& 2$ & 3329 & 1278 & \\
\hline & & $\& 3$ & 3744 & 1271 & \\
\hline & & $\& 4$ & 4916 & 1258 & \\
\hline & & $\& 3$ & 2972 & 1293 & \\
\hline & & $\& 4$ & 3080 & 1288 & \\
\hline & & $\& 4$ & 3259 & 1284 & \\
\hline & & $\& 5$ & 2453 & 1294 & \\
\hline & & $\& 5$ & 3392 & 1276 & \\
\hline & & $\& 5$ & 3729 & 1271 & \\
\hline & & $\& 5$ & 4195 & 1267 & \\
\hline & & erage & 3550 & 1279 & \\
\hline & \multicolumn{4}{|c|}{$k_{\text {PCET }} \tau_{0}=k_{\text {PCET }}^{\prime} \tau_{0}+k_{\mathrm{SV}}^{\prime}=1279+390=1670$} & \\
\hline & \multicolumn{4}{|c|}{$\Delta G^{\circ}{ }_{H-b o n d}(\mathrm{kcal} / \mathrm{mol})=-\mathrm{RT} \ln K_{\mathrm{A}}=-4.84$} & \\
\hline & \multicolumn{4}{|c|}{$\ln \left[k_{\text {PCET }}\left(M^{-1} s^{-1}\right)\right]=\ln \left[k_{\text {PCET }} \tau_{0} / \tau_{0}\right]=20.36$} & \\
\hline & \multicolumn{4}{|c|}{$\tau_{0}=1.8 \mu \mathrm{s}$} & \\
\hline & \multicolumn{4}{|c|}{ error in $\Delta G^{\circ}{ }_{\text {-bond }}(\mathrm{kcal} / \mathrm{mol})=0.20$} & \\
\hline
\end{tabular}




\subsection{Entry 4.}

Amide does not quench the luminescence of oxidant * Ir (III), therefore, the following equation is used:

$$
\frac{I_{0}}{I}=1+\left(k^{\prime}{ }_{S V}+k_{o b s} \tau_{0} S_{0}\right) A_{0}
$$

Table S2.4.1. Luminescence quenching data with $S_{0}=0.006 \mathrm{M}, A_{0}=0.0002 \mathrm{M}$. $S_{0}$ fixed at $0.006 \mathrm{M}$. $A_{0}$ varied from 0.0001 to $0.0003 \mathrm{M}$.

\begin{tabular}{ccccccc}
\hline $\mathbf{A}_{0} / \mathbf{M}$ & $\mathbf{S}_{0} \mathbf{A}_{0} / \mathbf{M}^{2}$ & I/au 1 & I/au 2 & I/au 3 & I/au ave & $I_{0} / \mathbf{I}$ \\
0 & 0 & & & & & 1 \\
0.0001 & 0.0000006 & 696 & 696 & 697 & 696.3333 & 1.016324 \\
0.00015 & 0.0000009 & 691 & 691 & 691 & 691 & 1.024168 \\
0.0002 & 0.0000012 & 687 & 686 & 684 & 685.6667 & 1.032134 \\
0.00025 & 0.0000015 & 680 & 680 & 681 & 680.3333 & 1.040225 \\
0.0003 & 0.0000018 & 675 & 675 & 675 & 675 & 1.048444 \\
\hline
\end{tabular}

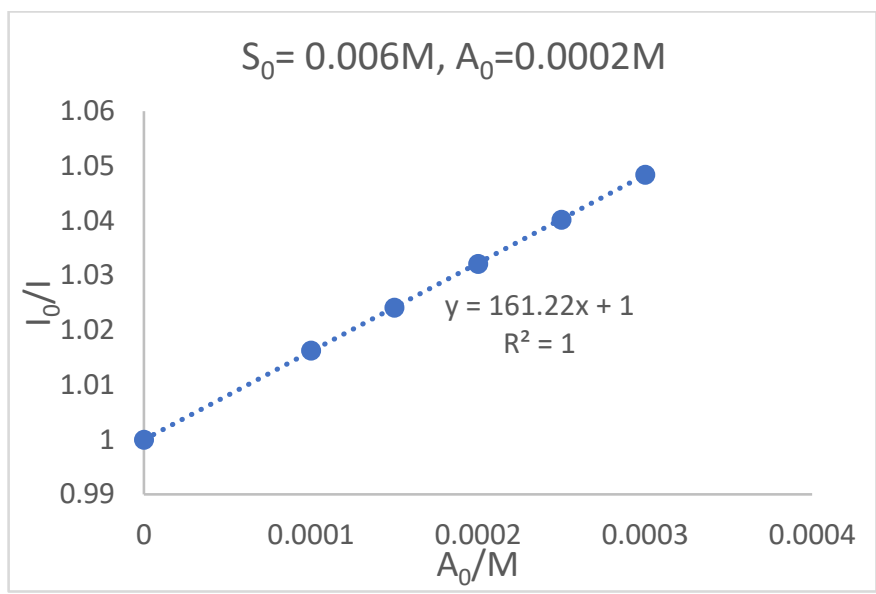

Figure S2.4.1. Stern-Volmer plot of luminescence data in Table S2.4.1.

$I_{0}$ was extrapolated from the five data points.

$k^{\prime}{ }_{S V}+k_{o b s} \tau_{0} S_{0}=161.2,148+k_{o b s} \tau_{0} \times 0.006=161.2, k_{o b s} \tau_{0}=2200$

Substitute $K_{\mathrm{A}}=3550$ (from entry 3 ), $k_{o b s} \tau_{0}=2200$ into equation a

$$
\begin{gathered}
0.006+0.0002=\frac{0.006 \times 0.0002 \times 2200}{k_{P C E T} \tau_{0}}+\frac{k_{P C E T}^{\prime} \tau_{0}}{2200}-\frac{1}{3550} \\
k_{\text {PCET } \tau_{0}=14}^{\prime}
\end{gathered}
$$


Table S2.4.2. Luminescence quenching data with $S_{0}=0.012 M, A_{0}=0.0004 M$. $S_{0}$ fixed at $0.012 M$. $A_{0}$ varied from 0.0001 to $0.0005 \mathrm{M}$.

\begin{tabular}{ccccccc}
\hline$A_{0} / \mathbf{M}$ & $\mathbf{S}_{0} A_{0} / M^{2}$ & I/au 1 & I/au 2 & I/au 3 & I/au ave & $I_{0} / \mathbf{I}$ \\
0 & 0 & & & & & 1 \\
0.0001 & 0.0000012 & 733 & 734 & 734 & 733.6667 & 1.015448 \\
0.0002 & 0.0000024 & 722 & 722 & 722 & 722 & 1.031856 \\
0.0003 & 0.0000036 & 711 & 711 & 711 & 711 & 1.04782 \\
0.0004 & 0.0000048 & 699 & 702 & 700 & 700.3333 & 1.063779 \\
0.0005 & 0.000006 & 690 & 690 & 691 & 690.3333 & 1.079189 \\
\hline
\end{tabular}

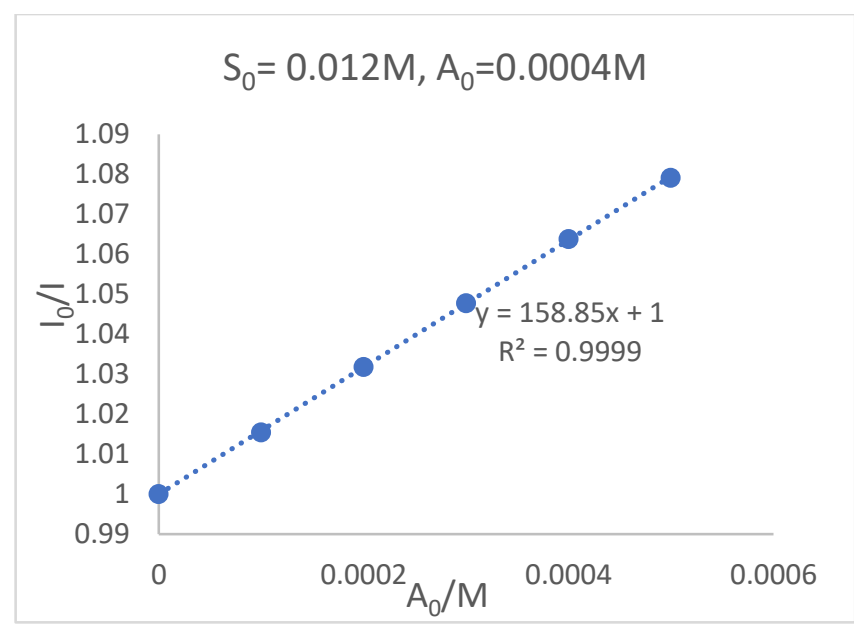

Figure S2.4.2. Stern-Volmer plot of luminescence data in Table S2.4.2.

$I_{0}$ was extrapolated from the five data points.

$k^{\prime}{ }_{S V}+k_{o b s} \tau_{0} S_{0}=158.9,148+k_{o b s} \tau_{0} \times 0.012=158.9, k_{o b s} \tau_{0}=910$

Substitute $K_{\mathrm{A}}=3550$ (from entry 3 ), $k_{o b s} \tau_{0}=910$ into equation a

$$
\begin{aligned}
& 0.012+0.0004=\frac{0.012 \times 0.0004 \times 910}{k_{P C E T} \tau_{0}}+\frac{k_{P C E T}^{\prime} \tau_{0}}{910}-\frac{1}{3550} \\
& k_{\text {PCET }}^{\prime} \tau_{0}=11 \\
& k_{\text {PCET }}^{\prime} \text { average } \tau_{0}=12.5 \\
& k_{\mathrm{PCET}} \tau_{0}=k_{\mathrm{PCET}}^{\prime} \tau_{0}+k_{\mathrm{SV}}^{\prime}=12.5+148=160 \\
& \Delta G^{\circ}{ }_{\mathrm{H} \text {-bond }}(\mathrm{kcal} / \mathrm{mol})=-\mathrm{RT} \ln K_{\mathrm{A}}=-4.84 \\
& \ln \left[k_{\text {PCET }}\left(M^{-1} \mathrm{~s}^{-1}\right)\right]=\ln \left[k_{\text {PCET }} / \tau_{0} / \tau_{0}\right]=18.0 \\
& \tau_{0}=2.3 \mu \mathrm{s}
\end{aligned}
$$


error in $\Delta G^{\circ}{ }_{\text {-bond }}(\mathrm{kcal} / \mathrm{mol})=0.20$

$$
\text { error in } \ln \left[k_{\operatorname{PCET}}\left(\mathrm{M}^{-1} \mathrm{~S}^{-1}\right)\right]=0.10
$$

\subsection{Entry 5.}

Amide does not quench the luminescence of oxidant * Ir (III), therefore, the following equation is used:

$$
\frac{I_{0}}{I}=1+\left(k_{S V}^{\prime}+k_{o b s} \tau_{0} S_{0}\right) A_{0}
$$

Table. S2.5.1. $S_{0}$ fixed at $0.005 \mathrm{M}, \mathrm{A}_{0}$ varied from 0.000125 to $0.00025 \mathrm{M}$

\begin{tabular}{ccccccc}
\hline $\mathbf{A}_{0} / \mathbf{M}$ & $\mathrm{S}_{0} \mathbf{A}_{0} / \mathbf{M}^{2}$ & I/au 1 & I/au 2 & I/au 3 & I/au ave & $I_{0} / \mathbf{I}$ \\
0 & 0 & & & & & 1 \\
0.000125 & $6.25 \mathrm{E}-07$ & 775 & 774 & 774 & 774.3333 & 1.211365 \\
0.00015 & 0.00000075 & 749 & 749 & 750 & 749.3333 & 1.251779 \\
0.000175 & $8.75 \mathrm{E}-07$ & 722 & 722 & 723 & 722.3333 & 1.298569 \\
0.0002 & 0.000001 & 698 & 697 & 700 & 698.3333 & 1.343198 \\
0.000225 & $1.125 \mathrm{E}-06$ & 677 & 677 & 677 & 677 & 1.385524 \\
0.00025 & 0.00000125 & 657 & 658 & 660 & 658.3333 & 1.42481 \\
\hline
\end{tabular}

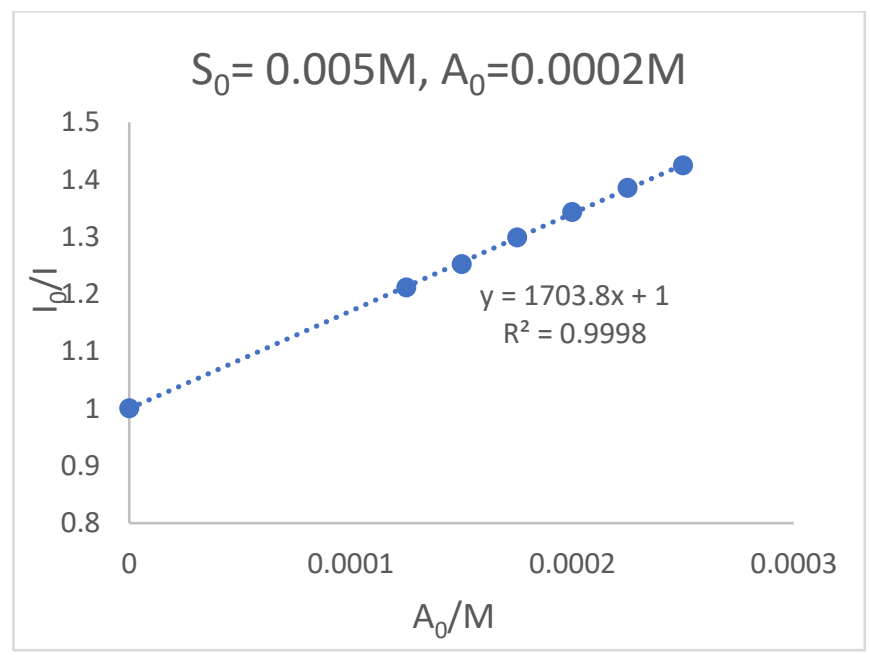

Figure S2.5.1. Stern-Volmer plot of luminescence data in Table S2.5.1.

$I_{0}$ was extrapolated from the six data points.

$$
k^{\prime}{ }_{S V}+k_{o b s} \tau_{0} S_{0}=1704
$$




$$
390+k_{o b s} \tau_{0} \times 0.006=1704, k_{o b s} \tau_{0}=262800
$$

Table. S2.5.2. $S_{0}$ fixed at $0.008 \mathrm{M}, \mathrm{A}_{0}$ varied from 0.000125 to $0.00025 \mathrm{M}$

\begin{tabular}{|c|c|c|c|c|c|c|}
\hline$A_{0} / M$ & $S_{0} A_{0} / M^{2}$ & I/au 1 & I/au 2 & I/au 3 & I/au ave & $I_{0} / 1$ \\
\hline 0 & 0 & & & & & 1 \\
\hline 0.000125 & 0.000001 & 913 & 913 & 913 & 913 & 1.221796 \\
\hline 0.00015 & 0.0000012 & 881 & 880 & 880 & 880.3333 & 1.267134 \\
\hline 0.000175 & 0.0000014 & 852 & 851 & 850 & 851 & 1.310811 \\
\hline 0.0002 & 0.0000016 & 820 & 822 & 824 & 822 & 1.357056 \\
\hline 0.000225 & 0.0000018 & 797 & 796 & 798 & 797 & 1.399624 \\
\hline 0.00025 & 0.000002 & 773 & 773 & 774 & 773.3333 & 1.442457 \\
\hline
\end{tabular}

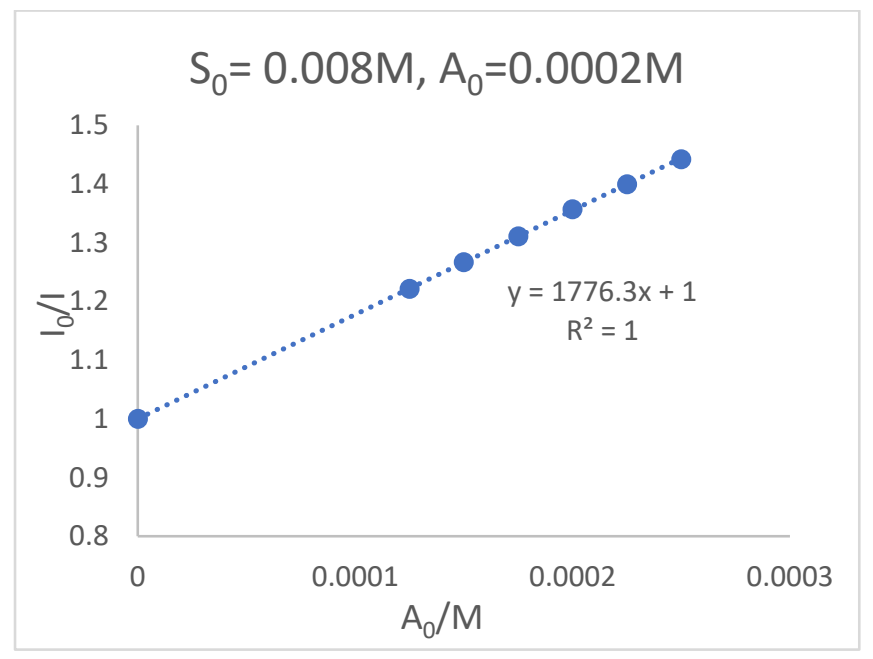

Figure S2.5.2. Stern-Volmer plot of luminescence data in Table S2.5.2.

$I_{0}$ was extrapolated from the six data points.

$$
\begin{gathered}
k^{\prime}{ }_{S V}+k_{o b s} \tau_{0} S_{0}=1776 \\
390+k_{o b s} \tau_{0} \times 0.006=1776, k_{o b s} \tau_{0}=173250
\end{gathered}
$$


Table. S2.5.3. $\mathrm{S}_{0}$ fixed at $0.01 \mathrm{M}, \mathrm{A}_{0}$ varied from 0.000125 to $0.00025 \mathrm{M}$

\begin{tabular}{ccccccc}
\hline $\mathbf{A}_{0} / \mathbf{M}$ & $\mathrm{S}_{0} \mathbf{A}_{0} / \mathbf{M}^{2}$ & I/au 1 & I/au 2 & I/au 3 & I/au ave & $I_{0} / \mathbf{I}$ \\
0 & 0 & & & & & 1 \\
0.000125 & 0.00000125 & 886 & 886 & 886 & 886 & 1.224605 \\
0.00015 & 0.0000015 & 853 & 854 & 854 & 853.6667 & 1.270988 \\
0.000175 & 0.00000175 & 825 & 828 & 825 & 826 & 1.313559 \\
0.0002 & 0.000002 & 800 & 798 & 798 & 798.6667 & 1.358514 \\
0.000225 & 0.00000225 & 775 & 774 & 774 & 774.3333 & 1.401205 \\
0.00025 & 0.0000025 & 751 & 751 & 752 & 751.3333 & 1.444099 \\
\hline
\end{tabular}

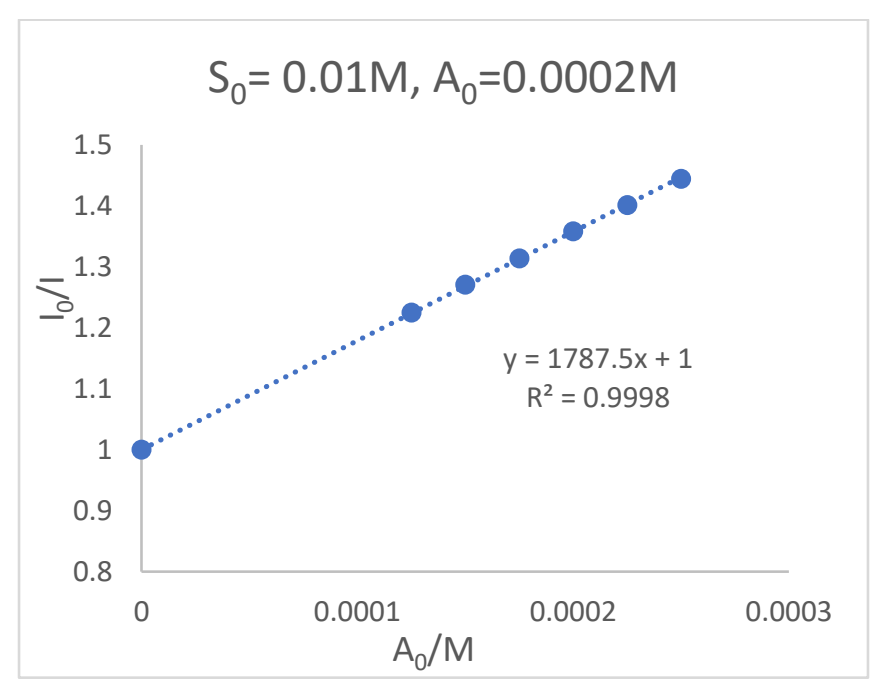

Figure S2.5.3. Stern-Volmer plot of luminescence data in Table S2.5.3.

$I_{0}$ was extrapolated from the six data points.

$$
\begin{gathered}
k^{\prime}{ }_{S V}+k_{o b s} \tau_{0} S_{0}=1787 \\
390+k_{o b s} \tau_{0} \times 0.006=1787, k_{o b s} \tau_{0}=141000
\end{gathered}
$$


Table 2.5.4. Solutions to Key Equation a and Entry 5 in Table S2.

\begin{tabular}{|c|c|c|c|c|c|}
\hline & $S_{0}(M)$ & $A_{0}(M)$ & $S_{0} A_{0}\left(M^{2}\right)$ & $S_{0}+A_{0}(M)$ & $\boldsymbol{k}_{\text {obs }} \tau_{0}$ \\
\hline 1 & 0.005 & 0.0002 & 0.000001 & 0.0052 & 262800 \\
\hline 2 & 0.008 & 0.0002 & $1.6 \mathrm{E}-06$ & 0.0082 & 173250 \\
\hline \multirow[t]{11}{*}{3} & 0.01 & 0.0002 & 0.000002 & 0.0102 & 141000 \\
\hline & & & $K_{\mathrm{A}} / \mathrm{M}^{-1}$ & $k_{\text {РСET }}^{\prime} \mathrm{T}_{0} / \mathrm{M}^{-1}$ & \\
\hline & & $1 \& 2$ & 1451 & 1502 & \\
\hline & & $1 \& 3$ & 1318 & 1520 & \\
\hline & & $2 \& 3$ & 1401 & 1512 & \\
\hline & & average & 1400 & 1511 & \\
\hline & \multicolumn{4}{|c|}{$k_{\mathrm{PCET}} \tau_{0}=k_{\text {PCET }}^{\prime} \tau_{0}+k_{\mathrm{SV}}^{\prime}=1511+390=1900$} & \\
\hline & \multicolumn{4}{|c|}{$\Delta G^{\circ}{ }_{\text {H-bond }}(\mathrm{kcal} / \mathrm{mol})=-\mathrm{RT} \ln K_{\mathrm{A}}=-4.28$} & \\
\hline & \multicolumn{4}{|c|}{$\ln \left[k_{\text {PCET }}\left(M^{-1} \mathrm{~S}^{-1}\right)\right]=\ln \left[k_{\text {PCET }} T_{0} / \tau_{0}\right]=20.78$} & \\
\hline & \multicolumn{4}{|c|}{$\tau_{0}=1.8 \mu \mathrm{s}$} & \\
\hline & \multicolumn{4}{|c|}{ error in $\Delta G^{\circ}{ }_{\text {H-bond }}(\mathrm{kcal} / \mathrm{mol})=0.02$} & \\
\hline
\end{tabular}

\subsection{Entry 6 .}

Amide does not quench the luminescence of oxidant ${ }^{*} \operatorname{Ir}(\mathrm{III})$, therefore, the following equation is used:

$$
\frac{I_{0}}{I}=1+\left(k^{\prime}{ }_{S V}+k_{o b s} \tau_{0} S_{0}\right) A_{0}
$$

Table S2.6.1. Luminescence quenching data with $S_{0}=0.02 \mathrm{M}, A_{0}=0.0005 \mathrm{M}$. $S_{0}$ fixed at $0.02 \mathrm{M}$. $A_{0}$ varied from 0.00025 to $0.00075 \mathrm{M}$.

\begin{tabular}{ccccccc}
\hline $\mathbf{A}_{0} / \mathbf{M}$ & $\mathbf{S}_{\mathbf{0}} \mathbf{A}_{0} / \mathbf{M}^{2}$ & I/au 1 & I/au 2 & I/au 3 & I/au ave & $I_{0} / \mathbf{I}$ \\
0 & 0 & & & & & 1 \\
0.00025 & 0.000005 & 667 & 667 & 667 & 667 & 1.04078 \\
0.000375 & 0.0000075 & 654 & 655 & 654 & 654.3333 & 1.060927 \\
0.0005 & 0.00001 & 642 & 642 & 643 & 642.3333 & 1.080747 \\
0.000625 & 0.0000125 & 631 & 631 & 631 & 631 & 1.100158 \\
0.00075 & 0.000015 & 620 & 620 & 620 & 620 & 1.119677 \\
\hline
\end{tabular}




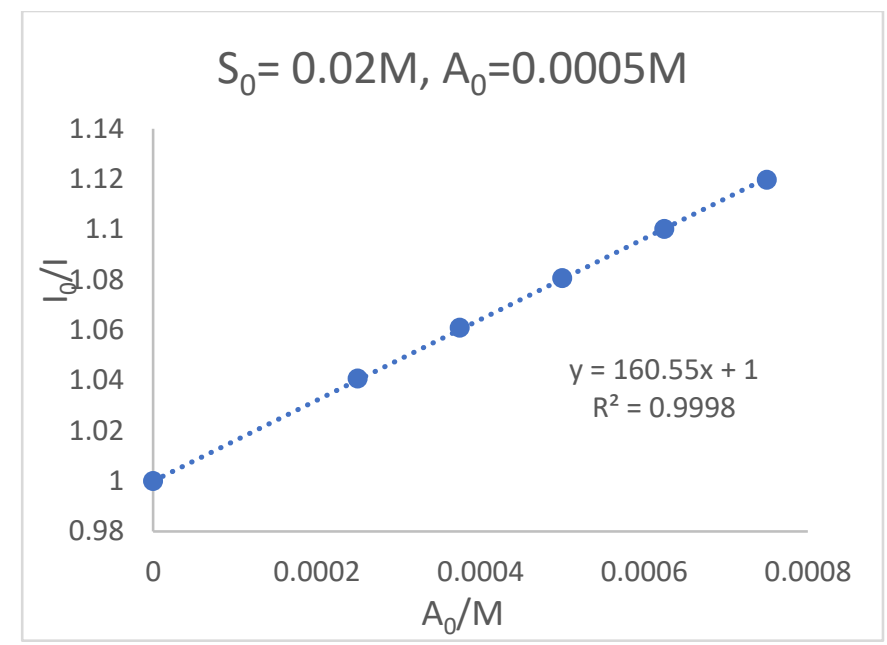

Figure S2.6.1. Stern-Volmer plot of luminescence data in Table S2.6.1.

$I_{0}$ was extrapolated from the five data points.

$k_{S V}^{\prime}+k_{o b s} \tau_{0} S_{0}=161,148+k_{o b s} \tau_{0} \times 0.006=161, k_{o b s} \tau_{0}=650$

Substitute $K_{\mathrm{A}}=1390$ (from entry 5), $k_{o b s} \tau_{0}=650$ into equation a

$$
\begin{gathered}
0.02+0.0005=\frac{0.02 \times 0.0005 \times 650}{k_{P C E T} \tau_{0}}+\frac{k_{P C E T}^{\prime} \tau_{0}}{650}-\frac{1}{1390} \\
k_{\text {PCET } \tau_{0}=13.5}^{\prime}
\end{gathered}
$$

Table S2.6.2. Luminescence quenching data with $S_{0}=0.02 \mathrm{M}, A_{0}=0.0002 \mathrm{M}$. $S_{0}$ fixed at $0.02 \mathrm{M}$. $A_{0}$ varied from 0.00006 to $0.00032 \mathrm{M}$.

\begin{tabular}{ccccccc}
\hline$A_{0} / \mathbf{M}$ & $\mathrm{S}_{0} A_{0} / M^{2}$ & I/au 1 & I/au 2 & I/au 3 & I/au ave & $I_{0} / \mathbf{I}$ \\
0 & 0 & & & & & 1 \\
0.000063375 & $1.2675 E-06$ & 823 & 824 & 822 & 823 & 1.010936 \\
0.00012675 & $2.535 E-06$ & 813 & 813 & 813 & 813 & 1.02337 \\
0.000190125 & $3.8025 E-06$ & 805 & 805 & 805 & 805 & 1.03354 \\
0.0002535 & 0.00000507 & 797 & 797 & 797 & 797 & 1.043915 \\
0.000316875 & $6.3375 E-06$ & 789 & 788 & 788 & 788.3333 & 1.055391 \\
\hline
\end{tabular}




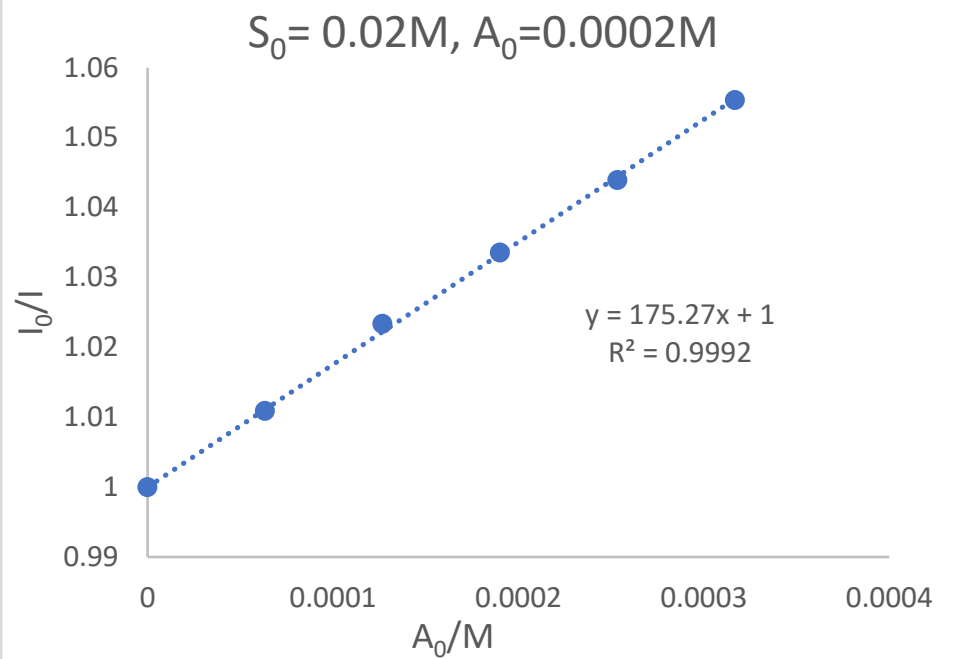

Figure S2.6.2. Stern-Volmer plot of luminescence data in Table S2.6.2.

$I_{0}$ was extrapolated from the five data points.

$k^{\prime}{ }_{S V}+k_{o b s} \tau_{0} S_{0}=175,148+k_{o b s} \tau_{0} \times 0.006=175, k_{o b s} \tau_{0}=1350$

Substitute $K_{\mathrm{A}}=1390$ (from entry 5), $k_{o b s} \tau_{0}=1350$ into equation a

$$
\begin{aligned}
& 0.02+0.0002=\frac{0.02 \times 0.0002 \times 1350}{k_{P C E T} \tau_{0}}+\frac{k_{P C E T}^{\prime} \tau_{0}}{1350}-\frac{1}{1390} \\
& k_{\mathrm{PCET}}^{\prime} \tau_{0}=27.6 \\
& k_{\text {PCET }}^{\prime} \text { average } \tau_{0}=21 \\
& k_{\mathrm{PCET}} \mathrm{T}_{0}=k_{\mathrm{PCET}}^{\prime} \tau_{0}+k_{\mathrm{SV}}^{\prime}=21+148=170 \\
& \Delta G^{\circ}{ }_{\text {H-bond }}(\mathrm{kcal} / \mathrm{mol})=-\mathrm{RT} \ln K_{\mathrm{A}}=-4.28 \\
& \ln \left[k_{\text {PCET }}\left(\mathrm{M}^{-1} \mathrm{~S}^{-1}\right)\right]=\ln \left[k_{\text {PCET }} \tau_{0} / \tau_{0}\right]=18.13 \\
& \tau_{0}=2.3 \mu \mathrm{s} \\
& \text { error in } \Delta G^{\circ}{ }^{H-b o n d}(\mathrm{kcal} / \mathrm{mol})=0.02 \\
& \text { error in } \ln \left[k_{\text {PСET }}\left(\mathrm{M}^{-1} \mathrm{~s}^{-1}\right)\right]=0.05
\end{aligned}
$$




\subsection{Entry 7.}

Amide does not quench the luminescence of oxidant * Ir (III), therefore, the following equation is used:

$$
\frac{I_{0}}{I}=1+\left(k^{\prime}{ }_{S V}+k_{o b s} \tau_{0} S_{0}\right) A_{0}
$$

Table. S2.7.1. $\mathrm{S}_{0}$ fixed at $0.0025 \mathrm{M}, \mathrm{A}_{0}$ varied from 0.00005 to $0.00030 \mathrm{M}$

\begin{tabular}{ccccccc}
\hline$A_{0} / M$ & $S_{0} A_{0} / M^{2}$ & I/au 1 & I/au 2 & I/au 3 & I/au ave & $I_{0} / I$ \\
0 & 0 & & & & & 1 \\
0.0001 & 0.00000025 & 238 & 239 & 240 & 239 & 1.435146 \\
0.00015 & 0.000000375 & 206 & 207 & 208 & 207 & 1.657005 \\
0.0002 & 0.0000005 & 185 & 183 & 184 & 184 & 1.86413 \\
0.00025 & 0.000000625 & 166 & 166 & 167 & 166.3333 & 2.062124 \\
0.0003 & 0.00000075 & 151 & 151 & 151 & 151 & 2.271523 \\
\hline
\end{tabular}

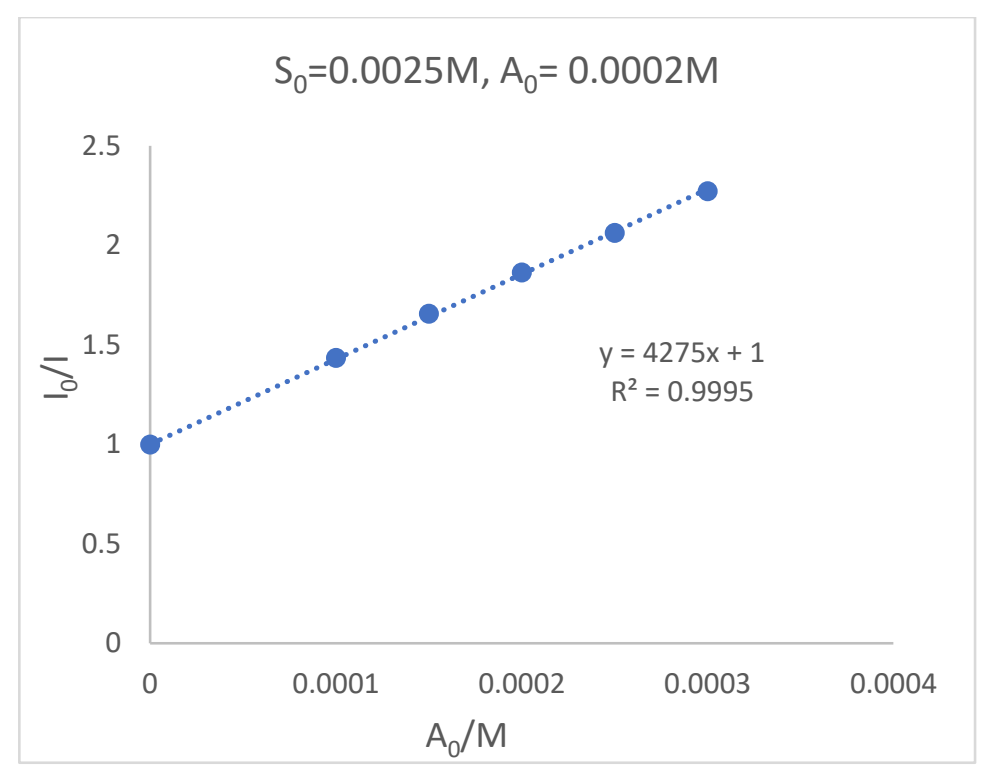

Figure S2.7.1. Stern-Volmer plot of luminescence data in Table S2.7.1.

$I_{0}$ was extrapolated from the five data points.

$$
\begin{gathered}
k^{\prime}{ }_{S V}+k_{o b s} \tau_{0} S_{0}=4275 \\
390+k_{o b s} \tau_{0} \times 0.0025=4275, k_{o b s} \tau_{0}=1554000
\end{gathered}
$$


Table. S2.7.2. $S_{0}$ fixed at $0.0039 \mathrm{M}, \mathrm{A}_{0}$ varied from 0.00005 to $0.00030 \mathrm{M}$

\begin{tabular}{ccccccc}
\hline $\mathrm{A}_{0} / \mathrm{M}$ & $\mathrm{S}_{0} \mathrm{~A}_{0} / \mathrm{M}^{2}$ & I/au 1 & I/au 2 & I/au 3 & I/au ave & $\begin{array}{c}\mathrm{I}_{0} / \mathrm{I} \\
0\end{array}$ \\
0 & 0 & & & & & 1 \\
$4.87805 \mathrm{E}-05$ & $1.90244 \mathrm{E}-07$ & 299 & 300 & 300 & 299.6667 & 1.199666 \\
$9.7561 \mathrm{E}-05$ & $3.80488 \mathrm{E}-07$ & 238 & 239 & 239 & 238.6667 & 1.506285 \\
0.000146341 & $5.70732 \mathrm{E}-07$ & 213 & 213 & 212 & 212.6667 & 1.690439 \\
0.000195122 & $7.60976 \mathrm{E}-07$ & 188 & 189 & 189 & 188.6667 & 1.905477 \\
0.000243902 & $9.5122 \mathrm{E}-07$ & 169 & 170 & 170 & 169.6667 & 2.118861 \\
0.000292683 & $1.14146 \mathrm{E}-06$ & 152 & 152 & 153 & 152.3333 & 2.359956 \\
\hline
\end{tabular}

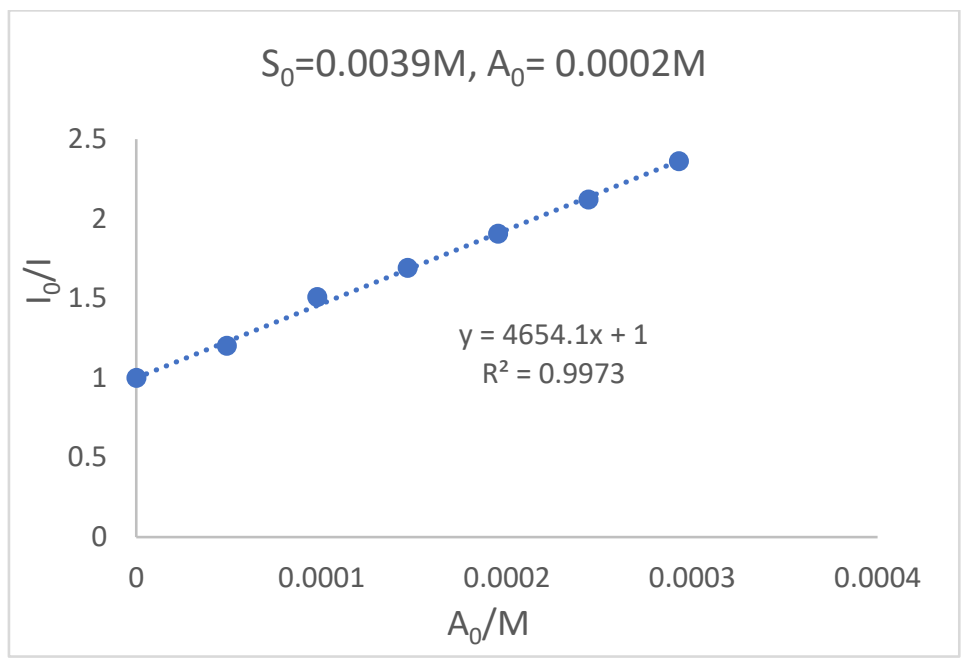

Figure S2.7.2. Stern-Volmer plot of luminescence data in Table S2.7.2.

$$
\begin{aligned}
& I_{0} \text { was extrapolated from the six data points. } \\
& \qquad k^{\prime}{ }_{S V}+k_{o b s} \tau_{0} S_{0}=4654 \\
& 390+k_{o b s} \tau_{0} \times 0.0039=4654, k_{o b s} \tau_{0}=1093000
\end{aligned}
$$


Table. S2.7.3. So fixed at $0.0050 \mathrm{M}$, Ao varied from 0.00005 to $0.00030 \mathrm{M}$

\begin{tabular}{ccccccc}
\hline$A_{0} / M$ & $S_{0} A_{0} / M^{2}$ & I/au 1 & I/au 2 & I/au 3 & I/au ave & $I_{0} / I$ \\
0 & 0 & & & & & 1 \\
0.0001 & 0.0000005 & 263 & 262 & 263 & 262.6667 & 1.492386 \\
0.00015 & 0.00000075 & 225 & 225 & 225 & 225 & 1.742222 \\
0.0002 & 0.000001 & 201 & 200 & 200 & 200.3333 & 1.956739 \\
0.00025 & 0.00000125 & 178 & 179 & 178 & 178.3333 & 2.198131 \\
0.0003 & 0.0000015 & 161 & 162 & 160 & 161 & 2.434783 \\
\hline
\end{tabular}

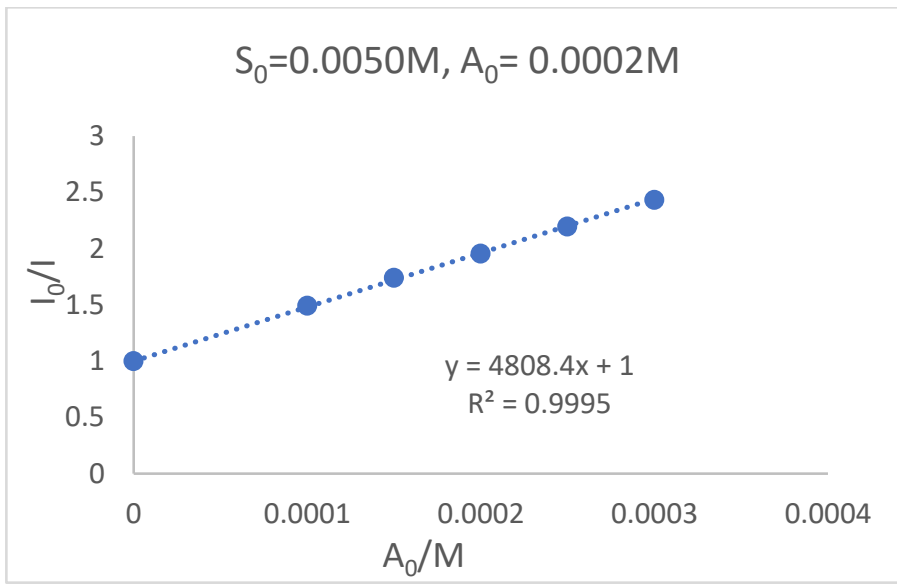

Figure S2.7.3. Stern-Volmer plot of luminescence data in Table S2.7.3.

$$
\begin{aligned}
& I_{0} \text { was extrapolated from the five data points. } \\
& \qquad k^{\prime}{ }_{S V}+k_{o b s} \tau_{0} S_{0}=4808 \\
& 390+k_{o b s} \tau_{0} \times 0.0050=4808, k_{o b s} \tau_{0}=883600
\end{aligned}
$$

Table. S2.7.4. $S_{0}$ fixed at $0.0076 \mathrm{M}, \mathrm{A}_{0}$ varied from 0.00005 to $0.00030 \mathrm{M}$

\begin{tabular}{ccccccc}
\hline $\mathbf{A}_{0} / \mathbf{M}$ & $\mathrm{S}_{0} \mathrm{~A}_{0} / \mathrm{M}^{2}$ & I/au 1 & I/au 2 & I/au 3 & I/au ave & $\begin{array}{c}\mathrm{I} / \mathrm{I} \\
0.000000\end{array}$ \\
0 & & & & & & 1 \\
0.000048 & $3.61905 \mathrm{E}-07$ & 308 & 310 & 310 & 309.3333 & 1.244612 \\
0.000095 & $7.2381 \mathrm{E}-07$ & 252 & 252 & 254 & 252.6667 & 1.523747 \\
0.000143 & $1.08571 \mathrm{E}-06$ & 221 & 220 & 220 & 220.3333 & 1.747352 \\
0.000190 & $1.44762 \mathrm{E}-06$ & 197 & 198 & 197 & 197.3333 & 1.951014 \\
0.000238 & $1.80952 \mathrm{E}-06$ & 177 & 177 & 177 & 177 & 2.175141 \\
0.000286 & $2.17143 \mathrm{E}-06$ & 160 & 160 & 160 & 160 & 2.40625 \\
\hline
\end{tabular}




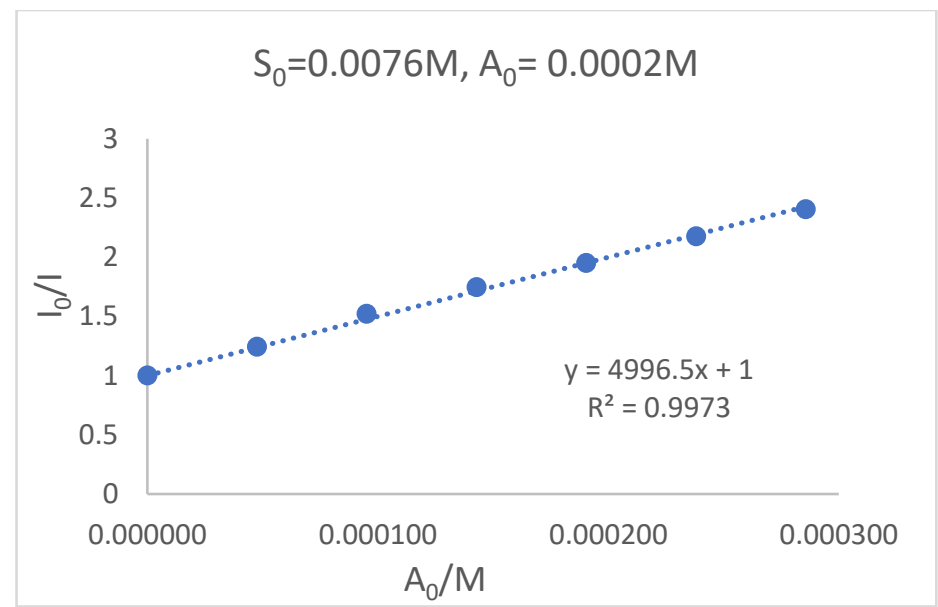

Figure S2.7.4. Stern-Volmer plot of luminescence data in Table S2.7.4.

$I_{0}$ was extrapolated from the six data points.

$$
\begin{gathered}
k_{S V}^{\prime}+k_{o b s} \tau_{0} S_{0}=4997 \\
390+k_{o b s} \tau_{0} \times 0.0050=4997, k_{o b s} \tau_{0}=606300
\end{gathered}
$$

Table 2.7.5. Solutions to Key Equation a and Entry 7 in Table S2.

\begin{tabular}{cccccc}
\hline & $\mathrm{S}_{0}(\mathrm{M})$ & $\mathrm{A}_{0}(\mathrm{M})$ & $\mathrm{S}_{0} \mathrm{~A}_{0}\left(\mathbf{M}^{2}\right)$ & $\mathrm{S}_{\mathbf{0}}+\mathrm{A}_{0}(\mathrm{M})$ & $\boldsymbol{k}_{\text {obs }} \boldsymbol{\tau}_{\mathbf{0}}$ \\
1 & 0.0025 & 0.0002 & $5 \mathrm{E}-07$ & 0.0027 & 1554000 \\
2 & 0.0039 & 0.0002 & $7.8 \mathrm{E}-07$ & 0.0041 & 1093000 \\
3 & 0.005 & 0.0002 & 0.000001 & 0.0052 & 883600 \\
4 & 0.0076 & 0.0002 & $1.52 \mathrm{E}-06$ & 0.0078 & 606300
\end{tabular}

\begin{tabular}{lcc} 
& $\boldsymbol{K}_{\mathrm{A}} / \mathbf{M}^{-1}$ & $\boldsymbol{k}_{\text {PCETT }}^{\prime} / \mathbf{M}^{-\mathbf{1}}$ \\
$1 \& 2$ & 1352 & 5109 \\
$1 \& 3$ & 1388 & 5078 \\
$1 \& 4$ & 1431 & 5042 \\
$2 \& 3$ & 1475 & 5039 \\
$2 \& 4$ & 1517 & 5018 \\
$3 \& 4$ & 1550 & 5009 \\
average & 1450 & 5050 \\
\hline
\end{tabular}

$$
\begin{gathered}
k_{\mathrm{PCET}} \tau_{0}=k_{\text {PCET }}^{\prime} \tau_{0}+k_{\text {SV }}^{\prime}=5050+390=5440 \\
\Delta G^{\circ}{ }_{\text {H-bond }}(\mathrm{kcal} / \mathrm{mol})=-\mathrm{RT} \ln K_{\mathrm{A}}=-4.33
\end{gathered}
$$




$$
\begin{gathered}
\ln \left[k_{\text {PCET }}\left(\mathrm{M}^{-1} \mathrm{~s}^{-1}\right)\right]=\ln \left[k_{\text {PCET }} / \tau_{0}\right]=21.83 \\
\tau_{0}=1.8 \mu \mathrm{s} \\
\text { error in } \Delta G^{\circ}{ }_{\text {H-bond }}(\mathrm{kcal} / \mathrm{mol})=0.08 \\
\text { error in } \ln \left[k_{\text {PCET }}\left(\mathrm{M}^{-1} \mathrm{~s}^{-1}\right)\right]=0.02
\end{gathered}
$$

\subsection{Entry 8.}

Amide does not quench the luminescence of oxidant * Ir (III), therefore, the following equation is used:

$$
\frac{I_{0}}{I}=1+\left(k^{\prime}{ }_{S V}+k_{o b s} \tau_{0} S_{0}\right) A_{0}
$$

Table. S2.8.1. $S_{0}$ fixed at $0.0050 \mathrm{M}, \mathrm{A}_{0}$ varied from 0.00005 to $0.00030 \mathrm{M}$

\begin{tabular}{ccccccc}
\hline $\mathbf{A}_{0} / \mathbf{M}$ & $\mathrm{S}_{0} \mathbf{A}_{0} / \mathbf{M}^{2}$ & I/au 1 & I/au 2 & I/au 3 & I/au ave & $I_{0} / \mathbf{I}$ \\
0 & 0 & & & & & 1 \\
0.00005 & 0.00000025 & 847 & 847 & 845 & 846.3333 & 1.021583 \\
0.00015 & 0.00000075 & 815 & 815 & 815 & 815 & 1.060859 \\
0.0002 & 0.000001 & 801 & 799 & 799 & 799.6667 & 1.081201 \\
0.00025 & 0.00000125 & 784 & 783 & 784 & 783.6667 & 1.103275 \\
0.0003 & 0.0000015 & 769 & 769 & 769 & 769 & 1.124317 \\
\hline
\end{tabular}




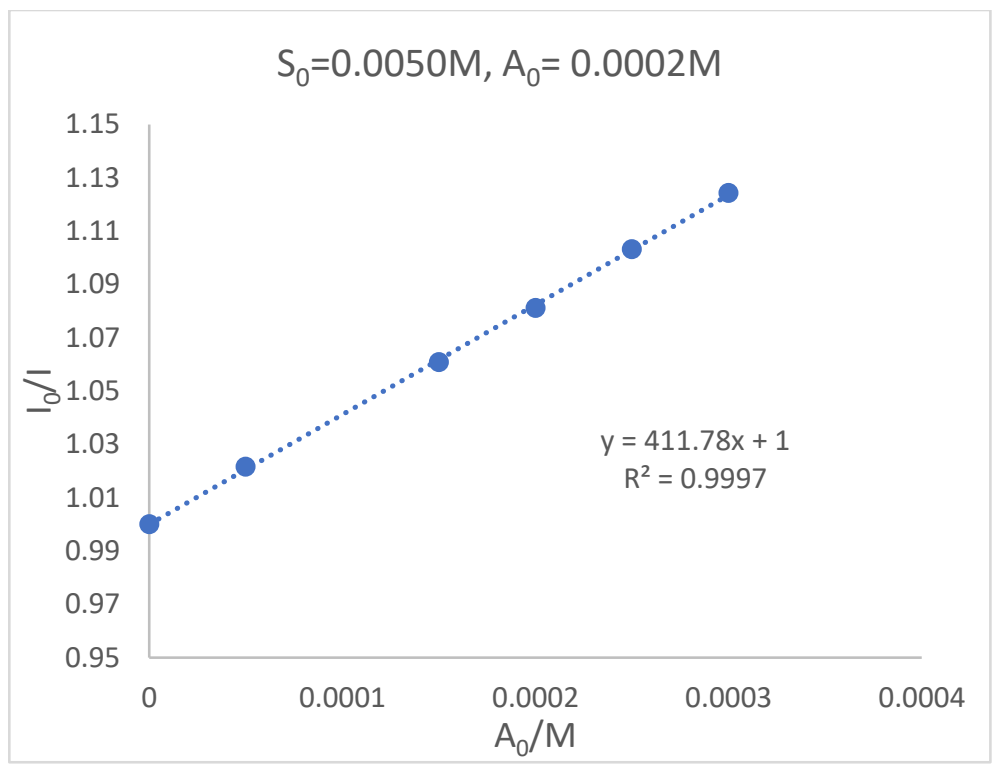

Figure S2.8.1. Stern-Volmer plot of luminescence data in Table S2.8.1.

$I_{0}$ was extrapolated from the five data points.

$$
\begin{gathered}
k^{\prime}{ }_{S V}+k_{o b s} \tau_{0} S_{0}=412 \\
148+k_{o b s} \tau_{0} \times 0.0050=412, k_{o b s} \tau_{0}=52800
\end{gathered}
$$

Substitute $K_{\mathrm{A}}=1450$ (from entry 7), $k_{o b s} \tau_{0}=52800$ into equation a

$$
\begin{gathered}
0.005+0.0002=\frac{0.005 \times 0.0002 \times 52800}{k_{P C E T} \tau_{0}}+\frac{k_{P C E T}^{\prime} \tau_{0}}{52800}-\frac{1}{1450} \\
k_{\text {PCET }}^{\prime} \tau_{0}=300
\end{gathered}
$$

\begin{tabular}{|c|c|c|c|c|c|c|}
\hline$A_{0} / M$ & $S_{0} A_{0} / M^{2}$ & I/au 1 & 1/au 2 & 1/au 3 & I/au ave & $\mathrm{I}_{0} / \mathrm{I}$ \\
\hline 0 & 0 & & & & & 1 \\
\hline 4.7619E-05 & 3.61905E-07 & 712 & 712 & 712 & 712 & 1.018258 \\
\hline 9.52381E-05 & 7.2381E-07 & 701 & 701 & 701 & 701 & 1.034237 \\
\hline 0.000142857 & $1.08571 \mathrm{E}-06$ & 690 & 690 & 689 & 689.6667 & 1.051232 \\
\hline 0.000190476 & $1.44762 \mathrm{E}-06$ & 680 & 678 & 678 & 678.6667 & 1.068271 \\
\hline 0.000285714 & 2.17143E-06 & 658 & 659 & 658 & 658.3333 & 1.101266 \\
\hline
\end{tabular}

Table. S2.8.2. $\mathrm{S}_{0}$ fixed at $0.0076 \mathrm{M}, \mathrm{A}_{0}$ varied from 0.00005 to $0.00030 \mathrm{M}$ 


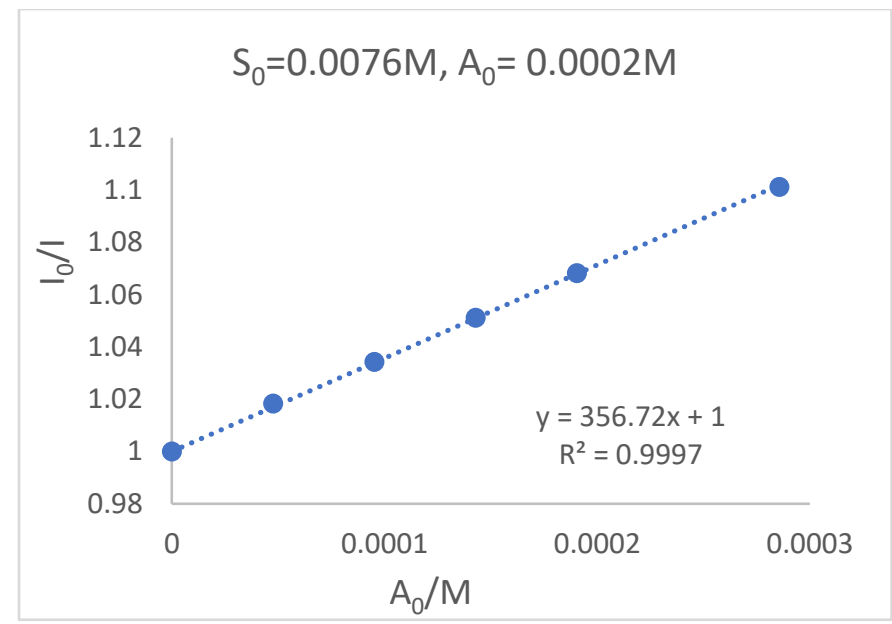

Figure S2.8.2. Stern-Volmer plot of luminescence data in Table S2.8.2.

$I_{0}$ was extrapolated from the five data points.

$$
\begin{gathered}
k^{\prime}{ }_{S V}+k_{o b s} \tau_{0} S_{0}=357 \\
148+k_{o b s} \tau_{0} \times 0.0076=357, k_{o b s} \tau_{0}=27500
\end{gathered}
$$

Substitute $K_{\mathrm{A}}=1450$ (from entry 7), $k_{o b s} \tau_{0}=27500$ into equation a

$$
\begin{aligned}
& 0.0076+0.0002=\frac{0.0076 \times 0.0002 \times 27500}{k_{P C E T} \tau_{0}}+\frac{k_{P C E T}^{\prime} \tau_{0}}{27500}-\frac{1}{1450} \\
& k_{\text {PCET }}^{\prime} \tau_{0}=234 \\
& k_{\text {PCET }}^{\prime} \text { average } \tau_{0}=270 \\
& k_{\text {PCET }} \tau_{0}=k_{\text {PCET }}^{\prime} \tau_{0}+k_{S V}^{\prime}=270+148=418 \\
& \Delta G^{\circ}{ }_{\text {H-bond }}(\mathrm{kcal} / \mathrm{mol})=-\mathrm{RT} \ln K_{\mathrm{A}}=-4.33 \\
& \ln \left[k_{\text {PCET }}\left(M^{-1} \mathrm{~S}^{-1}\right)\right]=\ln \left[k_{\text {PCET }} / \tau_{0} / \tau_{0}\right]=19.03 \\
& \tau_{0}=2.3 \mu \mathrm{s} \\
& \text { error in } \Delta G^{\circ}{ }_{\text {H-bond }}(\mathrm{kcal} / \mathrm{mol})=0.08 \\
& \text { error in } \ln \left[k_{\text {PCET }}\left(\mathrm{M}^{-1} \mathrm{~S}^{-1}\right)\right]=0.05
\end{aligned}
$$




\subsection{Entry 9.}

Thiol does not quench the luminescence of oxidant * Ir (III), therefore, the following equation is used:

$$
\frac{I_{0}}{I}=1+\left(k^{\prime}{ }_{S V}+k_{o b s} \tau_{0} S_{0}\right) A_{0}
$$

Table. S2.9.1. $\mathrm{S}_{0}$ fixed at $0.0025 \mathrm{M}, \mathrm{A}_{0}$ varied from 0.000025 to $0.000175 \mathrm{M}$

\begin{tabular}{|c|c|c|c|c|c|c|}
\hline$A_{0} / M$ & $\mathrm{~S}_{0} \mathrm{~A}_{0} / \mathrm{M}^{2}$ & I/au 1 & I/au 2 & I/au 3 & I/au ave & $\mathrm{I}_{0} / \mathrm{I}$ \\
\hline 0 & 0 & & & & & 1 \\
\hline 0.00005 & $1.25 \mathrm{E}-07$ & 338 & 336 & 337 & 337 & 1.284866 \\
\hline 0.000075 & $1.875 \mathrm{E}-07$ & 304 & 302 & 302 & 302.6667 & 1.430617 \\
\hline 0.0001 & $2.5 \mathrm{E}-07$ & 271 & 271 & 270 & 270.6667 & 1.599754 \\
\hline 0.000125 & $3.125 \mathrm{E}-07$ & 249 & 249 & 247 & 248.3333 & 1.743624 \\
\hline 0.00015 & $3.75 \mathrm{E}-07$ & 230 & 230 & 230 & 230 & 1.882609 \\
\hline 0.000175 & 4.375E-07 & 213 & 211 & 213 & 212.3333 & 2.039246 \\
\hline
\end{tabular}

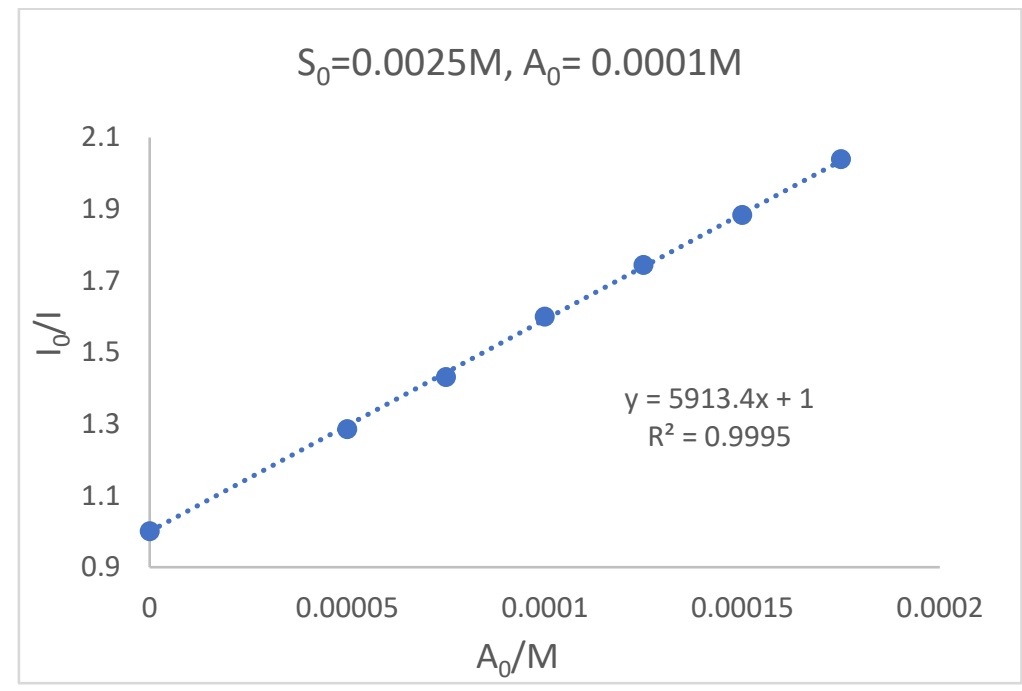

Figure S2.9.1. Stern-Volmer plot of luminescence data in Table S2.9.1.

$I_{0}$ was extrapolated from the six data points.

$$
\begin{gathered}
k^{\prime}{ }_{S V}+k_{o b s} \tau_{0} S_{0}=5913 \\
390+k_{o b s} \tau_{0} \times 0.0025=5913, k_{o b s} \tau_{0}=2209200
\end{gathered}
$$


Table. S2.9.2. $\mathrm{S}_{0}$ fixed at $0.0050 \mathrm{M}, \mathrm{A}_{0}$ varied from 0.00005 to $0.00015 \mathrm{M}$

\begin{tabular}{ccccccc}
\hline $\mathrm{A}_{0} / \mathrm{M}$ & $\mathrm{S}_{0} \mathrm{~A}_{0} / \mathrm{M}^{2}$ & I/au 1 & I/au 2 & I/au 3 & I/au ave & $\mathrm{I}_{0} / \mathrm{I}$ \\
0 & 0 & & & & & 1 \\
0.00005 & $2.5 \mathrm{E}-07$ & 378 & 376 & 376 & 376.6667 & 1.443451 \\
0.000075 & $3.75 \mathrm{E}-07$ & 329 & 321 & 323 & 324.3333 & 1.676362 \\
0.0001 & 0.0000005 & 292 & 292 & 292 & 292 & 1.861986 \\
0.000125 & $6.25 \mathrm{E}-07$ & 257 & 258 & 258 & 257.6667 & 2.110091 \\
0.00015 & $7.5 \mathrm{E}-07$ & 233 & 233 & 232 & 232.6667 & 2.336819 \\
\hline
\end{tabular}

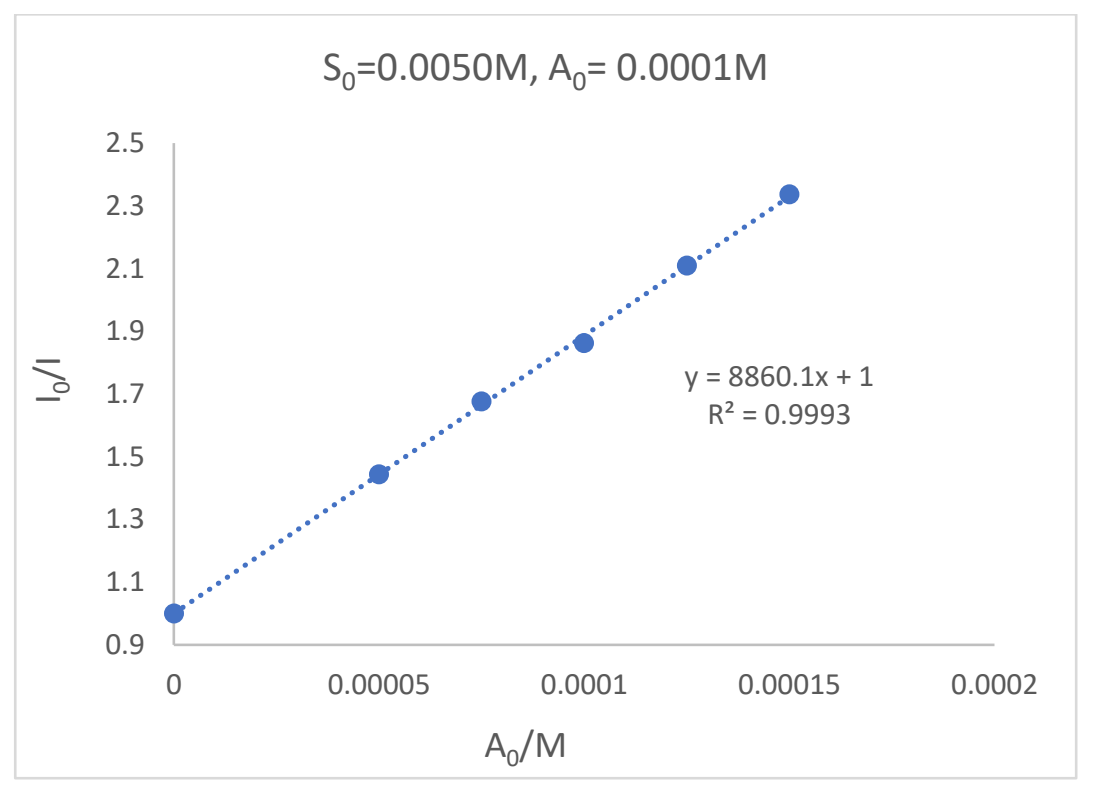

Figure S2.9.2. Stern-Volmer plot of luminescence data in Table S2.9.2.

$I_{0}$ was extrapolated from the five data points.

$$
\begin{gathered}
k^{\prime}{ }_{S V}+k_{o b s} \tau_{0} S_{0}=8860 \\
390+k_{o b s} \tau_{0} \times 0.0050=8860, k_{o b s} \tau_{0}=1691000
\end{gathered}
$$


Table. S2.9.3. $\mathrm{S}_{0}$ fixed at $0.010 \mathrm{M}, \mathrm{A}_{0}$ varied from 0.00005 to $0.00015 \mathrm{M}$

\begin{tabular}{ccccccc}
\hline $\mathrm{A}_{0} / \mathrm{M}$ & $\mathrm{S}_{0} \mathrm{~A}_{0} / \mathrm{M}^{2}$ & I/au 1 & I/au 2 & I/au 3 & I/au ave & $\mathrm{I}_{0} / \mathrm{I}$ \\
0 & 0 & & & & & 1 \\
0.00005 & 0.0000005 & 346 & 347 & 351 & 348 & 1.58046 \\
0.000075 & $7.5 \mathrm{E}-07$ & 297 & 296 & 296 & 296.3333 & 1.856018 \\
0.0001 & 0.000001 & 255 & 255 & 254 & 254.6667 & 2.159686 \\
0.000125 & $1.25 \mathrm{E}-06$ & 225 & 224 & 224 & 224.3333 & 2.451709 \\
0.00015 & 0.0000015 & 202 & 204 & 202 & 202.6667 & 2.713816 \\
\hline
\end{tabular}

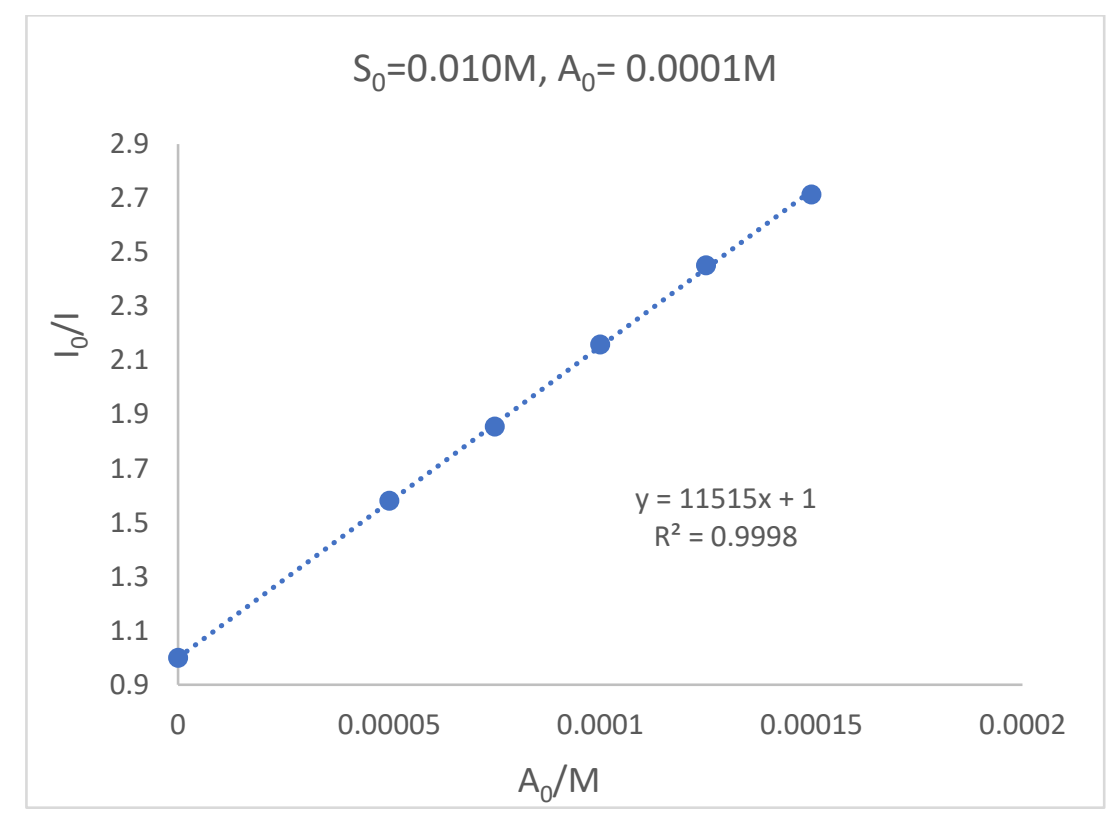

Figure S2.9.3. Stern-Volmer plot of luminescence data in Table S2.9.3.

$I_{0}$ was extrapolated from the five data points.

$$
\begin{gathered}
k_{S V}^{\prime}+k_{o b s} \tau_{0} S_{0}=11515 \\
390+k_{o b s} \tau_{0} \times 0.010=11515, k_{o b s} \tau_{0}=1112600
\end{gathered}
$$


Table. S2.9.4. $S_{0}$ fixed at $0.015 \mathrm{M}, \mathrm{A}_{0}$ varied from 0.00005 to $0.000125 \mathrm{M}$

\begin{tabular}{ccccccc}
\hline $\mathbf{A}_{0} / \mathbf{M}$ & $\mathrm{S}_{0} \mathrm{~A}_{0} / \mathbf{M}^{2}$ & I/au 1 & I/au 2 & I/au 3 & I/au ave & $\mathrm{I}_{\mathbf{0}} / \mathbf{I}$ \\
0 & 0 & & & & & 1 \\
0.00005 & $7.5 \mathrm{E}-07$ & 333 & 333 & 333 & 333 & 1.66967 \\
0.000075 & $1.125 \mathrm{E}-06$ & 289 & 287 & 287 & 287.6667 & 1.932793 \\
0.0001 & 0.0000015 & 239 & 239 & 239 & 239 & 2.32636 \\
0.000125 & $1.875 \mathrm{E}-06$ & 213 & 212 & 214 & 213 & 2.610329 \\
\hline
\end{tabular}

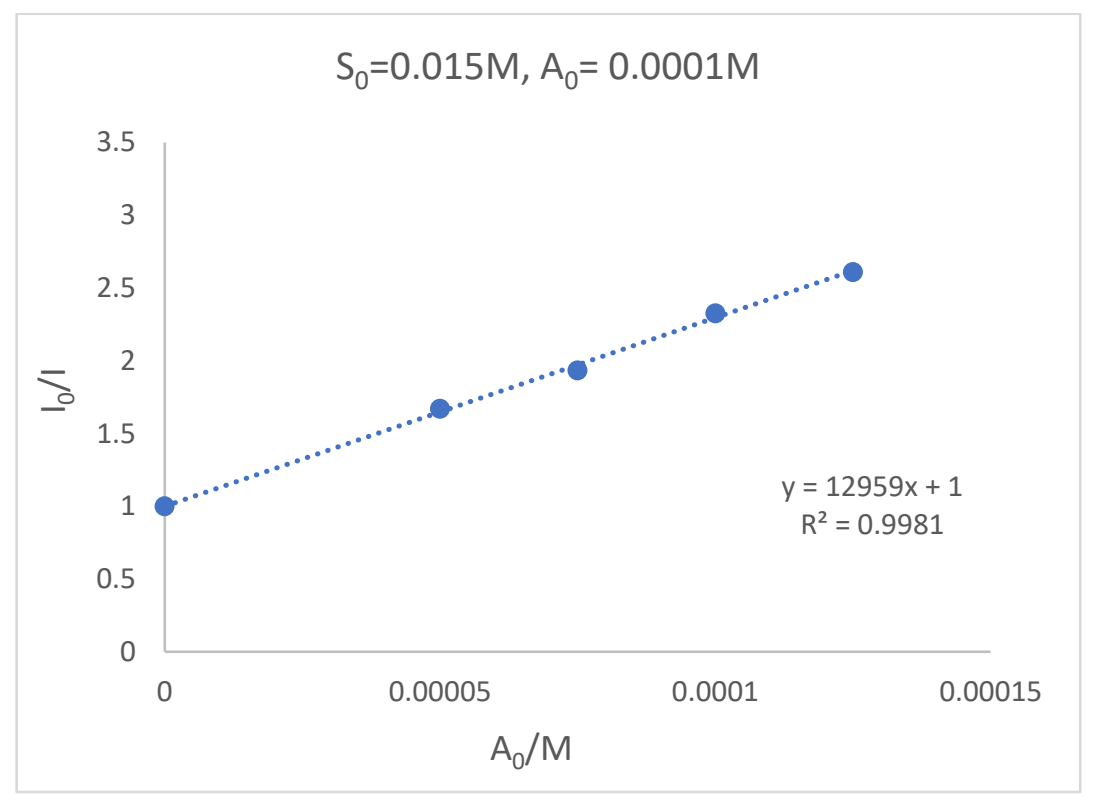

Figure S2.9.4. Stern-Volmer plot of luminescence data in Table S2.9.4.

$I_{0}$ was extrapolated from the four data points.

$$
\begin{gathered}
k^{\prime}{ }_{S V}+k_{o b s} \tau_{0} S_{0}=12959 \\
390+k_{o b s} \tau_{0} \times 0.010=12959, k_{o b s} \tau_{0}=838000
\end{gathered}
$$


Table. S2.9.5. $S_{0}$ fixed at $0.0188 \mathrm{M}, \mathrm{A}_{0}$ varied from 0.000075 to $0.0002 \mathrm{M}$

\begin{tabular}{ccccccc}
\hline $\mathbf{A}_{0} / \mathbf{M}$ & $\mathrm{S}_{0} \mathrm{~A}_{0} / \mathrm{M}^{2}$ & I/au 1 & I/au 2 & I/au 3 & I/au ave & $\begin{array}{c}\text { Io/I } \\
0\end{array}$ \\
0 & 0 & & & & & 1 \\
0.000075 & $1.41 \mathrm{E}-06$ & 540 & 540 & 542 & 540.6667 & 1.991985 \\
0.0001 & $1.88 \mathrm{E}-06$ & 449 & 452 & 449 & 450 & 2.393333 \\
0.000125 & $2.35 \mathrm{E}-06$ & 393 & 390 & 391 & 391.3333 & 2.752129 \\
0.00015 & $2.82 \mathrm{E}-06$ & 350 & 347 & 347 & 348 & 3.094828 \\
0.0002 & $3.76 \mathrm{E}-06$ & 285 & 288 & 288 & 287 & 3.752613 \\
\hline
\end{tabular}

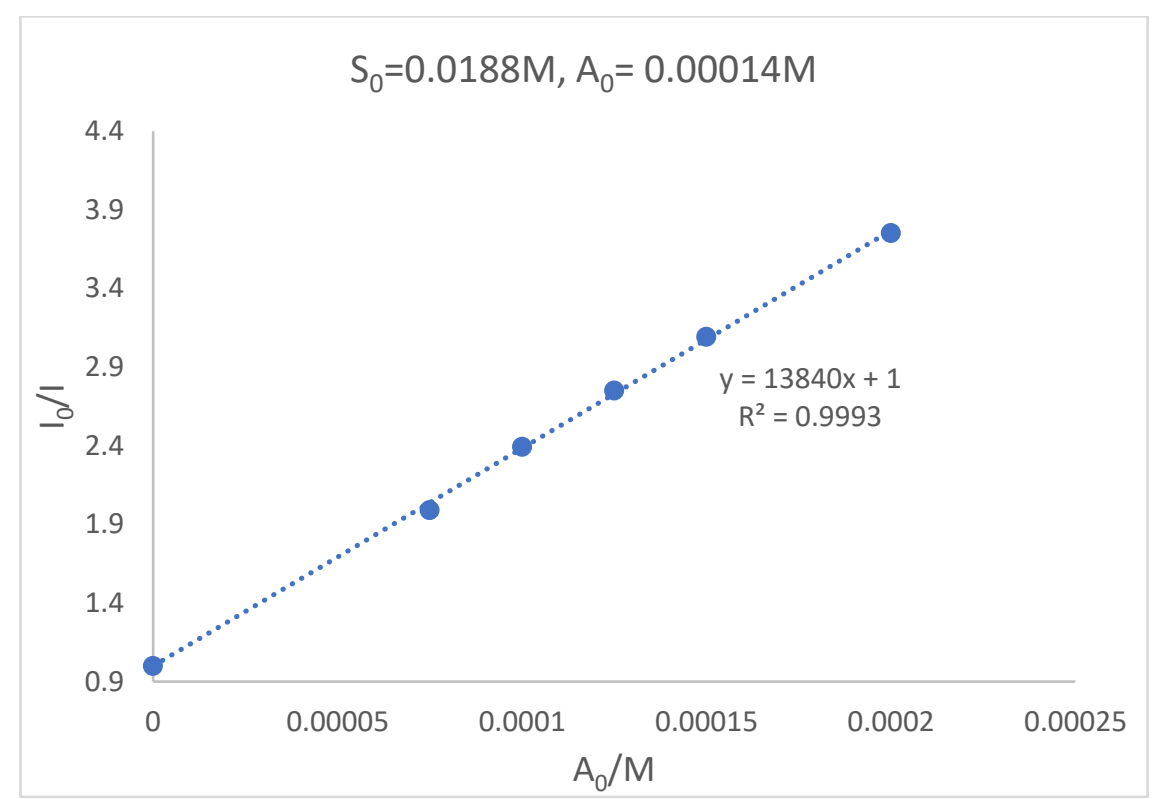

Figure S2.9.5. Stern-Volmer plot of luminescence data in Table S2.9.5.

$I_{0}$ was extrapolated from the four data points.

$$
\begin{gathered}
k^{\prime}{ }_{S V}+k_{o b s} \tau_{0} S_{0}=13840 \\
390+k_{o b s} \tau_{0} \times 0.010=13840, k_{o b s} \tau_{0}=715400
\end{gathered}
$$


Table 2.9.6. Solutions to Key Equation a and Entry 9 in Table S2.

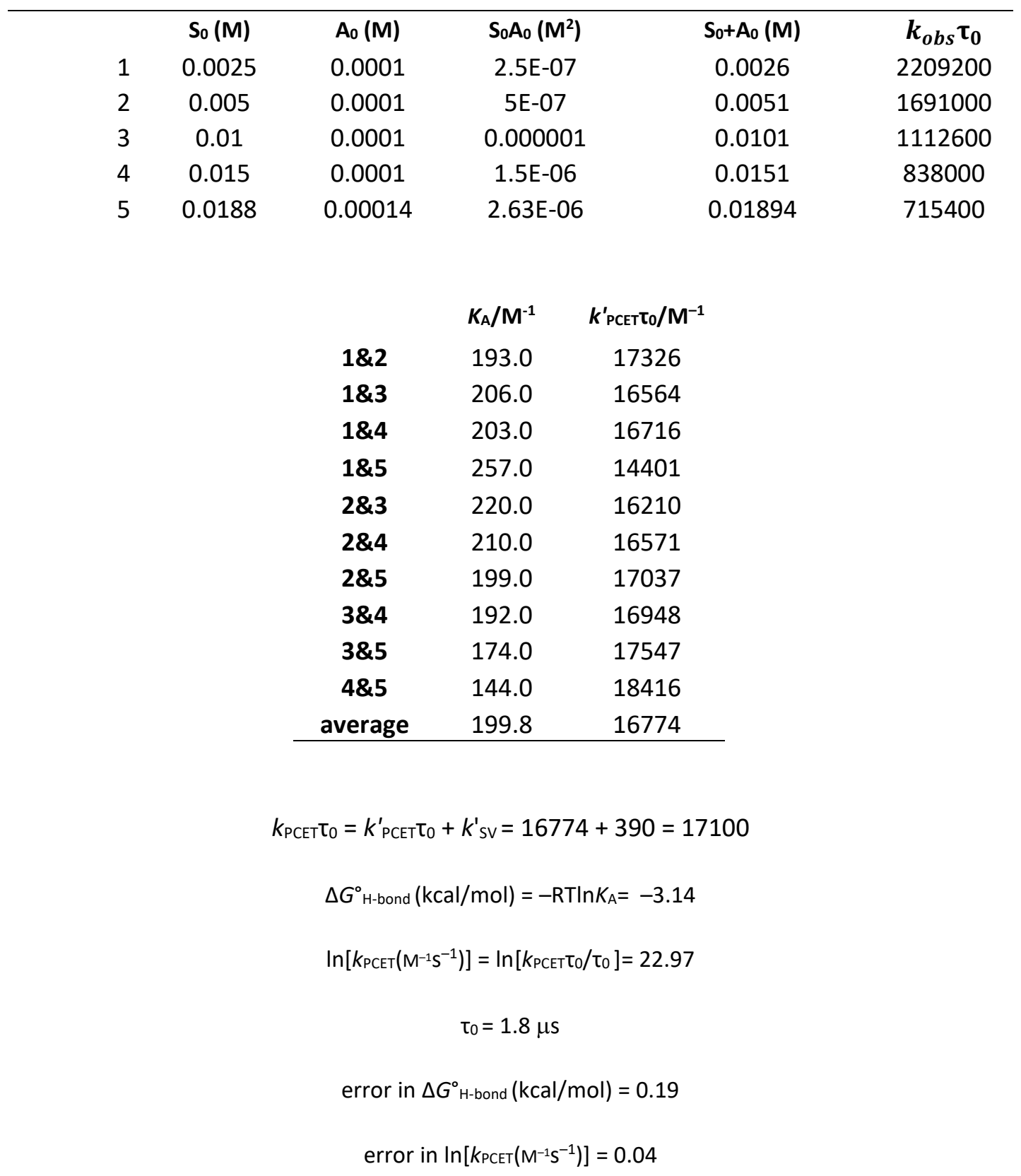




\subsection{Entry 10.}

Thiol does not quench the luminescence of oxidant * Ir (III), therefore, the following equation is used:

$$
\frac{I_{0}}{I}=1+\left(k^{\prime}{ }_{S V}+k_{o b s} \tau_{0} S_{0}\right) A_{0}
$$

Table. S2.10.1. $S_{0}$ fixed at $0.0050 \mathrm{M}, \mathrm{A}_{0}$ varied from 0.000025 to $0.000125 \mathrm{M}$

\begin{tabular}{ccccccc}
\hline $\mathbf{A}_{0} / \mathbf{M}$ & $\mathbf{S}_{0} \mathbf{A}_{0} / \mathbf{M}^{2}$ & I/au 1 & I/au 2 & I/au 3 & I/au ave & $\begin{array}{c}\text { Io/I } \\
0\end{array}$ \\
0 & & & & & & 1 \\
0.000025 & $1.25 \mathrm{E}-07$ & 515 & 515 & 515 & 515 & 1.106796 \\
0.00005 & $2.5 \mathrm{E}-07$ & 467 & 467 & 467 & 467 & 1.220557 \\
0.000075 & $3.75 \mathrm{E}-07$ & 430 & 431 & 430 & 430.3333 & 1.324555 \\
0.0001 & $5 \mathrm{E}-07$ & 399 & 398 & 398 & 398.3333 & 1.430962 \\
0.000125 & $6.25 \mathrm{E}-07$ & 373 & 373 & 372 & 372.6667 & 1.529517 \\
\hline
\end{tabular}

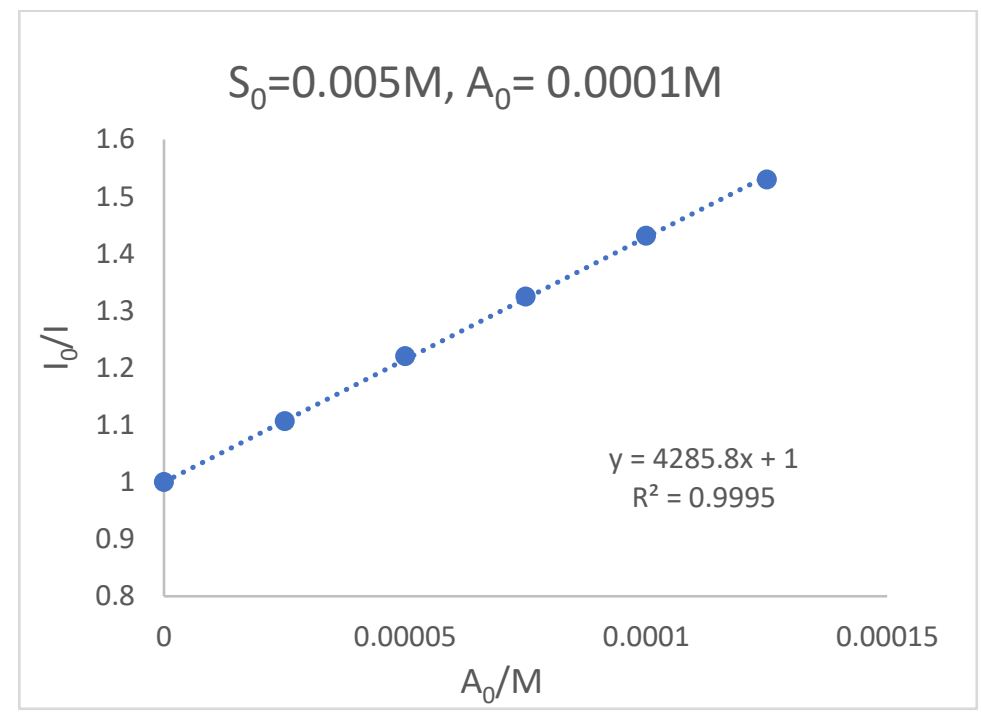

Figure S2.10.1. Stern-Volmer plot of luminescence data in Table S2.10.1.

$I_{0}$ was extrapolated from the five data points.

$$
\begin{gathered}
k^{\prime}{ }_{S V}+k_{o b s} \tau_{0} S_{0}=4286 \\
148+k_{o b s} \tau_{0} \times 0.005=4286, k_{o b s} \tau_{0}=827600
\end{gathered}
$$

Substitute $K_{\mathrm{A}}=200$ (from entry 7), $k_{o b s} \tau_{0}=827600$ into equation a

$$
0.0050+0.0001=\frac{0.0050 \times 0.0001 \times 827600}{k_{P C E T} \tau_{0}}+\frac{k_{P C E T}^{\prime} \tau_{0}}{827600}-\frac{1}{200}
$$




$$
k_{\mathrm{PCET}}^{\prime} \tau_{0}=8310
$$

Table. S2.10.2. $\mathrm{S}_{0}$ fixed at $0.0025 \mathrm{M}, \mathrm{A}_{0}$ varied from 0.00005 to $0.000125 \mathrm{M}$

\begin{tabular}{ccccccc}
\hline $\mathrm{A}_{0} / \mathrm{M}$ & $\mathrm{S}_{0} \mathrm{~A}_{0} / \mathrm{M}^{2}$ & I/au 1 & I/au 2 & I/au 3 & I/au ave & Io/I \\
0 & 0 & & & & & 1 \\
0.00005 & $2.5 \mathrm{E}-07$ & 727 & 730 & 728 & 728.3333 & 1.13135 \\
0.000075 & $3.75 \mathrm{E}-07$ & 686 & 685 & 684 & 685 & 1.20292 \\
0.0001 & $5 \mathrm{E}-07$ & 646 & 647 & 647 & 646.6667 & 1.274227 \\
0.000125 & $6.25 \mathrm{E}-07$ & 620 & 620 & 621 & 620.3333 & 1.328318 \\
\hline
\end{tabular}

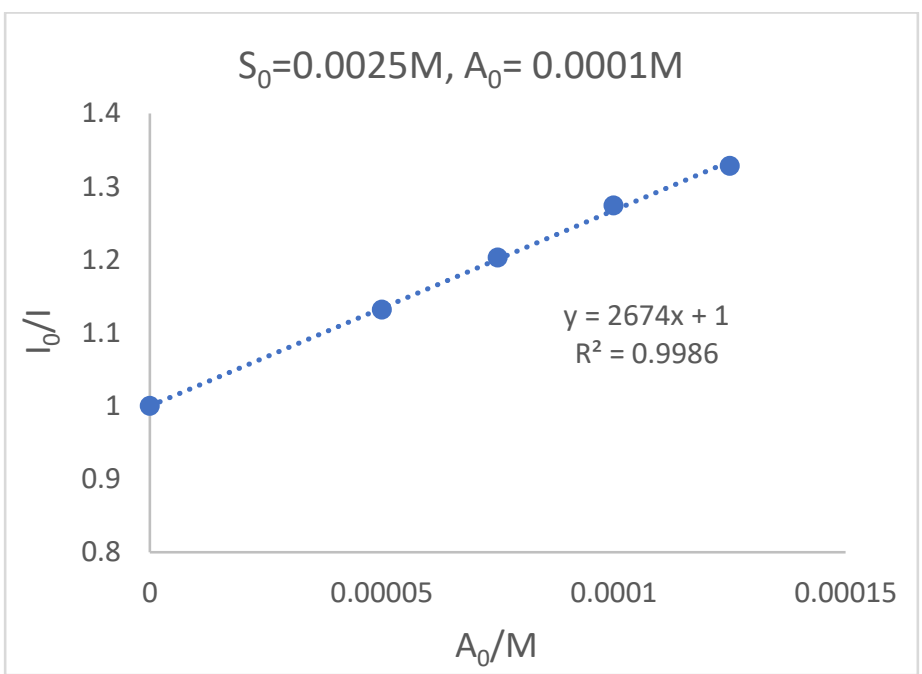

Figure S2.10.2. Stern-Volmer plot of luminescence data in Table S2.10.2.

$I_{0}$ was extrapolated from the four data points.

$$
\begin{gathered}
k^{\prime}{ }_{S V}+k_{o b s} \tau_{0} S_{0}=2674 \\
148+k_{o b s} \tau_{0} \times 0.0025=2674, k_{o b s} \tau_{0}=1010400
\end{gathered}
$$

Substitute $K_{\mathrm{A}}=200$ (from entry 7), $k_{o b s} \tau_{0}=1010400$ into equation a

$$
\begin{gathered}
0.0025+0.0001=\frac{0.0025 \times 0.0001 \times 1010400}{k_{P C E T} \tau_{0}}+\frac{k_{P C E T}^{\prime} \tau_{0}}{1010400}-\frac{1}{200} \\
k_{\text {PCET }}^{\prime} \tau_{0}=7580 \\
k_{\text {PCET }}^{\prime} \text { average } \tau_{0}=7950 \\
k_{\text {PCET }} \tau_{0}=k_{\text {PCET }}^{\prime} \tau_{0}+k_{\text {SV }}^{\prime}=7950+148=8090 \\
\Delta G^{\circ}{ }_{\text {H-bond }}(\mathrm{kcal} / \mathrm{mol})=-\mathrm{RT} \ln K_{\mathrm{A}}=-3.14
\end{gathered}
$$




$$
\begin{gathered}
\ln \left[k_{\text {PCET }}\left(\mathrm{M}^{-1} \mathrm{~S}^{-1}\right)\right]=\ln \left[k_{\text {PCET }} \mathrm{T}_{0} / \tau_{0}\right]=22.00 \\
\tau_{0}=2.3 \mu \mathrm{s} \\
\text { error in } \Delta G^{\circ}{ }_{\text {H-bond }}(\mathrm{kcal} / \mathrm{mol})=0.19 \\
\text { error in } \ln \left[k_{\text {PCET }}\left(\mathrm{M}^{-1} \mathrm{~s}^{-1}\right)\right]=0.20
\end{gathered}
$$

\subsection{Entry 11.}

Thiol dynamically quenches the luminescence of oxidant *Ir (III), therefore, the following equation is used:

$$
\frac{I_{0}}{I}=1+\frac{\left(k^{\prime}{ }_{S V}+k_{o b s} \tau_{0} \mathrm{~S}_{0}\right) \mathrm{A}_{0}}{1+k^{\prime \prime}{ }_{S V} \mathrm{~S}_{0}}
$$

where non-PCET quenching of oxidant by the thiol alone (without base) is given by the following equation:

$$
\mathrm{I} / \mathrm{I}=1+k^{\prime \prime} \mathrm{sv} S_{0}
$$

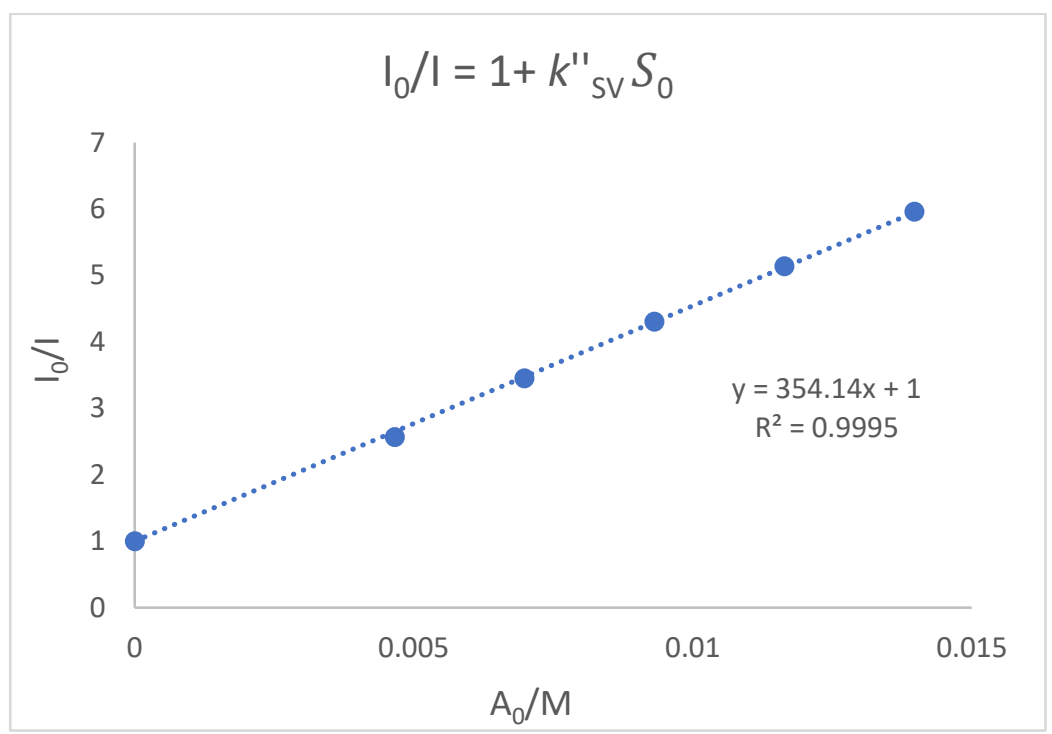

Figure S2.11. Stern-Volmer plot of oxidant *Ir (III) luminescence data of non-PCET quenching by thiol alone

$$
k_{S V}^{\prime \prime}=354
$$


Table. S2.11.1. $S_{0}$ fixed at $0.0010 \mathrm{M}, \mathrm{A}_{0}$ varied from 0.0003 to $0.0005 \mathrm{M}$

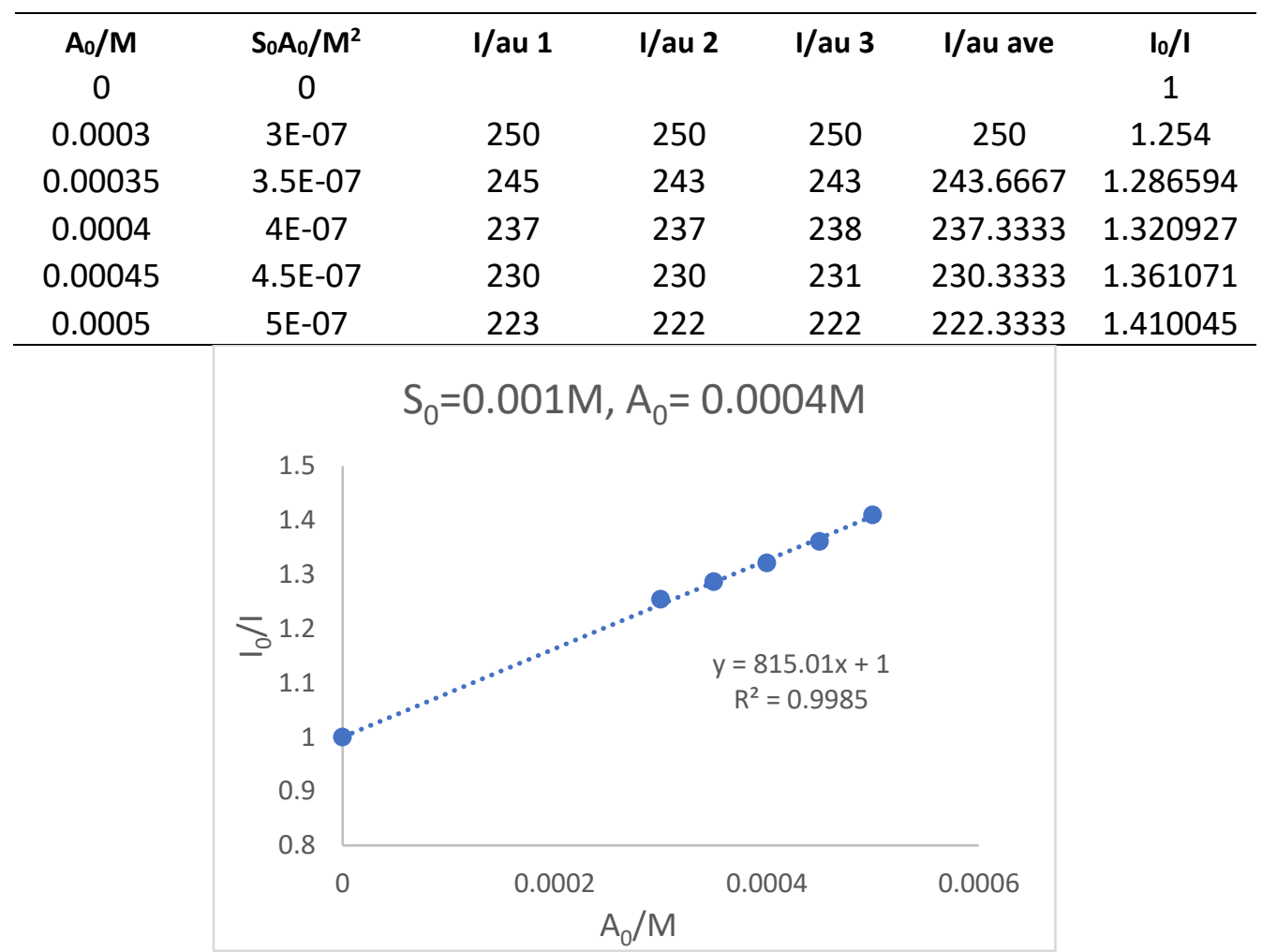

Figure S2.11.1. Stern-Volmer plot of luminescence data in Table S2.11.1.

$I_{0}$ was extrapolated from the five data points.

$$
\begin{gathered}
\frac{k_{s V}^{\prime \prime}+k_{o b s} \tau_{0} S_{0}}{1+k^{\prime}{ }_{d S V} S_{0}}=815 \\
k^{\prime \prime}{ }_{S V}+k_{o b s} \tau_{0} S_{0}=815 \times(1+354 \times 0.001)=1104 \\
390+0.001 k_{o b s} \tau_{0}=1104 \\
k_{o b s} \tau_{0}=714320
\end{gathered}
$$

\begin{tabular}{|c|c|c|c|c|c|c|}
\hline$A_{0} / M$ & $S_{0} A_{0} / M^{2}$ & I/au 1 & I/au 2 & I/au 3 & I/au ave & $10 / 1$ \\
\hline 0 & 0 & & & & & 1 \\
\hline 0.0001 & $5 \mathrm{E}-07$ & 70 & 70 & 70 & 70 & 1.12 \\
\hline 0.00015 & 7.5E-07 & 65 & 65 & 66 & 65.33333 & 1.2 \\
\hline 0.0002 & 0.000001 & 62 & 62 & 63 & 62.33333 & 1.257754 \\
\hline 0.00025 & $1.25 \mathrm{E}-06$ & 60 & 60 & 60 & 60 & 1.306667 \\
\hline 0.0003 & $1.5 \mathrm{E}-06$ & 56 & 57 & 57 & 56.66667 & 1.383529 \\
\hline
\end{tabular}

Table. S2.11.2. $S_{0}$ fixed at $0.0050 \mathrm{M}, \mathrm{A}_{0}$ varied from 0.0001 to $0.0003 \mathrm{M}$ 


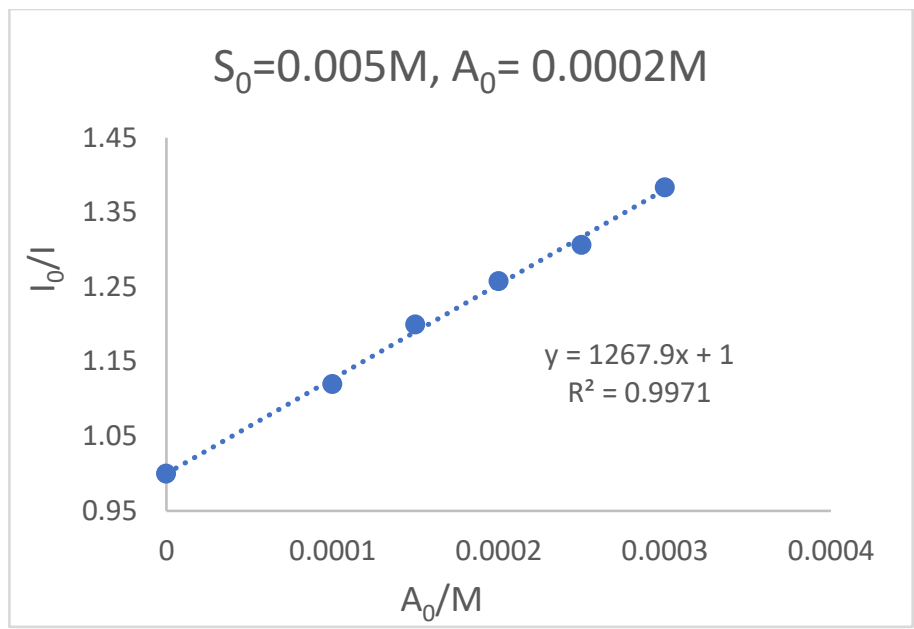

Figure S2.11.2. Stern-Volmer plot of luminescence data in Table S2.11.2.

$I_{0}$ was extrapolated from the five data points.

$$
\begin{gathered}
\frac{k^{\prime \prime}{ }_{S V}+k_{o b s} \tau_{0} \mathrm{~S}_{0}}{1+k^{\prime}{ }_{d S V} \mathrm{~S}_{0}}=1268 \\
k^{\prime \prime}{ }_{S V}+k_{o b s} \tau_{0} \mathrm{~S}_{0}=1268 \times(1+354 \times 0.005)=3512 \\
390+0.005 k_{o b s} \tau_{0}=3512 \\
k_{o b s} \tau_{0}=625740
\end{gathered}
$$

Table. S2.11.3. $\mathrm{S}_{0}$ fixed at $0.010 \mathrm{M}, \mathrm{A}_{0}$ varied from 0.0001 to $0.0003 \mathrm{M}$

\begin{tabular}{ccccccc}
\hline $\mathrm{A}_{0} / \mathrm{M}$ & $\mathrm{S}_{0} \mathrm{~A}_{0} / \mathrm{M}^{2}$ & I/au 1 & I/au 2 & I/au 3 & I/au ave & $\mathrm{I}_{0} / \mathbf{I}$ \\
0 & 0 & & & & & 1 \\
0.0001 & $1.5 \mathrm{E}-06$ & 120 & 119 & 119 & 119.3333 & 1.118715 \\
0.00015 & $2.25 \mathrm{E}-06$ & 112 & 112 & 112 & 112 & 1.191964 \\
0.0002 & 0.000003 & 107 & 106 & 106 & 106.3333 & 1.255486 \\
0.00025 & $3.75 \mathrm{E}-06$ & 101 & 102 & 102 & 101.6667 & 1.313115 \\
0.0003 & $4.5 \mathrm{E}-06$ & 98 & 98 & 98 & 98 & 1.362245 \\
\hline
\end{tabular}




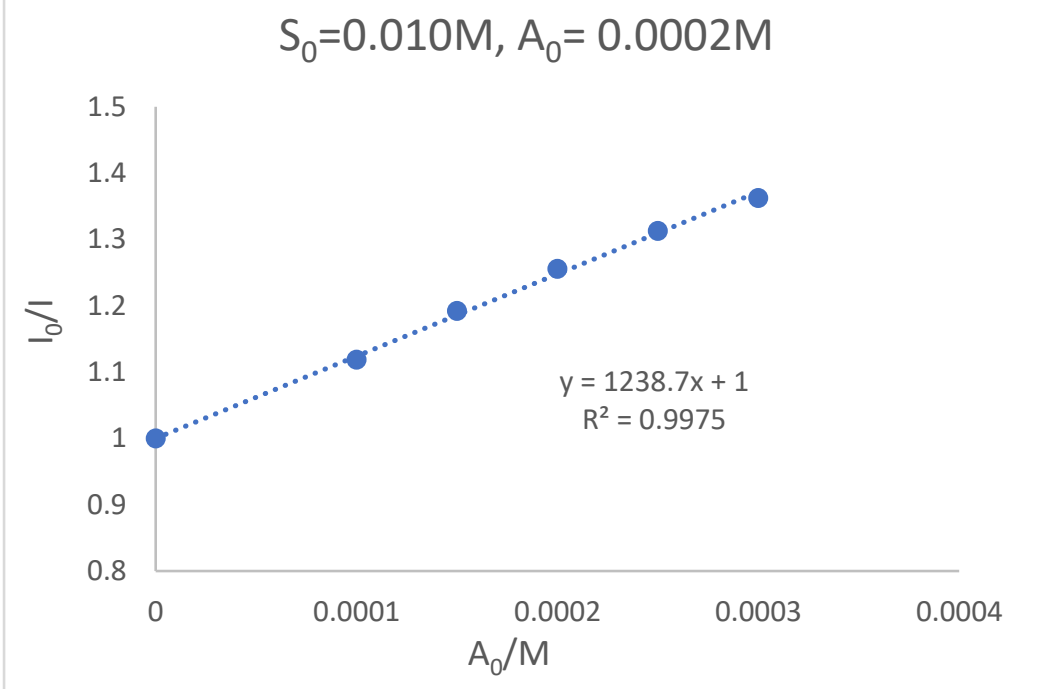

Figure S2.11.3. Stern-Volmer plot of luminescence data in Table S2.11.3.

$I_{0}$ was extrapolated from the five data points.

$$
\begin{gathered}
\frac{k_{S V}^{\prime \prime}+k_{o b s} \tau_{0} \mathrm{~S}_{0}}{1+k^{\prime}{ }_{d S V} \mathrm{~S}_{0}}=1239 \\
k_{S V}^{\prime \prime}+k_{o b s} \tau_{0} S_{0}=1239 \times(1+354 \times 0.01)=5625 \\
390+0.01 k_{o b s} \tau_{0}=5625 \\
k_{o b s} \tau_{0}=524740
\end{gathered}
$$

\begin{tabular}{|c|c|c|c|c|c|}
\hline & So (M) & $A_{0}(M)$ & $S_{0} A_{0}\left(M^{2}\right)$ & $S_{0}+A_{0}(M)$ & $k_{o b s} \tau_{0}$ \\
\hline 1 & 0.001 & 0.0004 & $4 \mathrm{E}-07$ & 0.0014 & 714320 \\
\hline 2 & 0.005 & 0.0002 & 0.000001 & 0.0052 & 625740 \\
\hline \multirow[t]{6}{*}{3} & 0.01 & 0.0002 & 0.000002 & 0.0102 & 524740 \\
\hline & & & $K_{\mathrm{A}} / \mathrm{M}^{-1}$ & $k_{\mathrm{PCET}}^{\prime} \mathrm{T}_{0} / \mathrm{M}^{-1}$ & \\
\hline & & $1 \& 2$ & 39 & 19084 & \\
\hline & & $1 \& 3$ & 44 & 17312 & \\
\hline & & $2 \& 3$ & 48 & 16171 & \\
\hline & & average & 44 & 17522 & \\
\hline
\end{tabular}

Table 2.11.4. Solutions to Key Equation a and Entry 11 in Table S2. 


$$
\begin{gathered}
k_{\text {PCET }} \tau_{0}=k_{\text {PCET }}^{\prime} \tau_{0}+k_{\text {SV }}^{\prime}=17522+390=17900 \\
\Delta G^{\circ}{ }_{\text {H-bond }}(\mathrm{kcal} / \mathrm{mol})=-\mathrm{RT} \ln K_{\mathrm{A}}=-2.24 \\
\ln \left[k_{\text {PCET }}\left(\mathrm{M}^{-1} \mathrm{~s}^{-1}\right)\right]=\ln \left[k_{\text {PCET }} / \tau_{0}\right]=23.07 \\
\tau_{0}=1.8 \mu \mathrm{s} \\
\text { error in } \Delta G^{\circ}{ }_{\text {H-bond }}(\mathrm{kcal} / \mathrm{mol})=0.07 \\
\text { error in } \ln \left[k_{\text {PCET }}\left(\mathrm{M}^{-1} \mathrm{~s}^{-1}\right)\right]=0.08
\end{gathered}
$$

\subsection{Entry 12.}

Thiol dynamically quenches the luminescence of oxidant *Ir (III), therefore, the following equation is used:

$$
\frac{I_{0}}{I}=1+\frac{\left(k^{\prime}{ }_{S V}+k_{o b s} \tau_{0} \mathrm{~S}_{0}\right) \mathrm{A}_{0}}{1+k^{\prime \prime}{ }_{S V} \mathrm{~S}_{0}}
$$

where non-PCET quenching of oxidant by the thiol alone (without base) is given by the following equation:

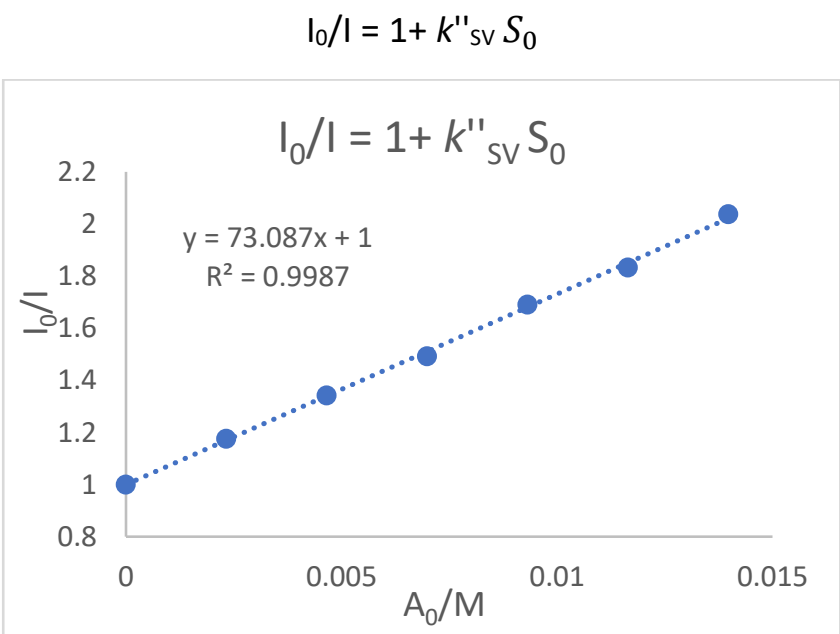

Figure S2.12. Stern-Volmer plot of oxidant *Ir (III) luminescence data of non-PCET quenching by thiol alone

$$
k_{S V}^{\prime \prime}=73
$$


Table. S2.12.1. $S_{0}$ fixed at $0.0050 \mathrm{M}, \mathrm{A}_{0}$ varied from 0.000025 to $0.00015 \mathrm{M}$

\begin{tabular}{|c|c|c|c|c|c|c|}
\hline$A_{0} / M$ & $S_{0} A_{0} / M^{2}$ & I/au 1 & I/au 2 & I/au 3 & I/au ave & $I_{0} / I$ \\
\hline 0 & 0 & & & & & 1 \\
\hline 0.000025 & $1.25 \mathrm{E}-07$ & 436 & 436 & 437 & 436.3333 & 1.047364 \\
\hline 0.00005 & $2.5 \mathrm{E}-07$ & 413 & 410 & 411 & 411.3333 & 1.111021 \\
\hline 0.000075 & $3.75 \mathrm{E}-07$ & 388 & 386 & 388 & 387.3333 & 1.179862 \\
\hline 0.0001 & 5E-07 & 370 & 370 & 370 & 370 & 1.235135 \\
\hline 0.00015 & $7.5 \mathrm{E}-07$ & 344 & 342 & 343 & 343 & 1.332362 \\
\hline
\end{tabular}

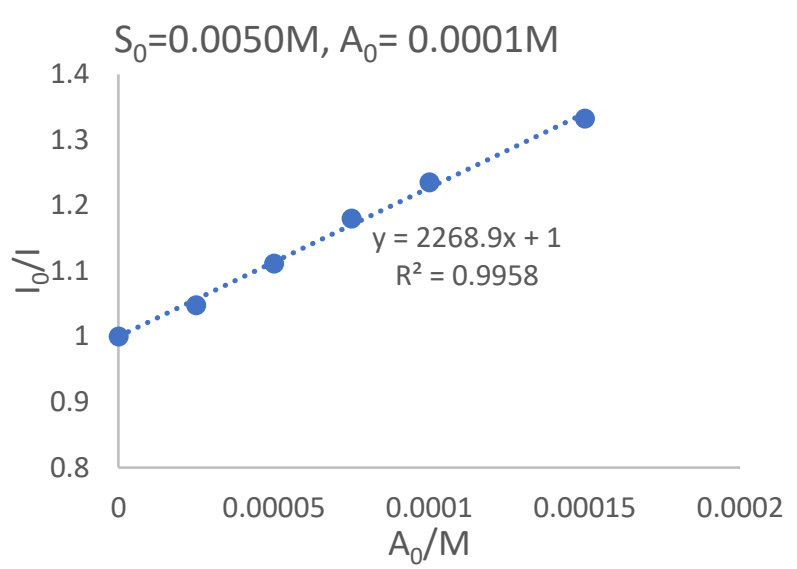

Figure S2.12.1. Stern-Volmer plot of luminescence data in Table S2.12.1.

$I_{0}$ was extrapolated from the five data points.

$$
\begin{gathered}
\frac{k^{\prime \prime}{ }_{S V}+k_{o b s} \tau_{0} \mathrm{~S}_{0}}{1+k^{\prime}{ }_{d S V} \mathrm{~S}_{0}}=2269 \\
k_{S V}^{\prime \prime}+k_{o b s} \tau_{0} \mathrm{~S}_{0}=2269 \times(1+73 \times 0.005)=3097 \\
148+0.005 k_{o b s} \tau_{0}=3097 \\
k_{o b s} \tau_{0}=589800
\end{gathered}
$$

Substitute $K_{\mathrm{A}}=44$ (from entry 11), $k_{o b s} \tau_{0}=589800$ into equation a

$$
\begin{gathered}
0.005+0.0001=\frac{0.005 \times 0.0001 \times 589800}{k_{P C E T} \tau_{0}}+\frac{k_{P C E T}^{\prime} \tau_{0}}{589800}-\frac{1}{44} \\
k_{\text {PCET }}^{\prime} \tau_{0}=16400
\end{gathered}
$$


Table. S2.12.2. $S_{0}$ fixed at $0.00166 \mathrm{M}, A_{0}$ varied from 0.0002 to $0.0003 \mathrm{M}$

\begin{tabular}{|c|c|c|c|c|c|c|}
\hline$A_{0} / M$ & $\mathrm{~S}_{0} \mathrm{~A}_{0} / \mathrm{M}^{2}$ & I/au 1 & I/au 2 & I/au 3 & I/au ave & $I_{0} / I$ \\
\hline 0 & 0 & & & & & 1 \\
\hline 0.0002 & 0.000001 & 639 & 640 & 639 & 639.3333 & 1.20438 \\
\hline 0.000225 & $1.13 \mathrm{E}-06$ & 626 & 622 & 630 & 626 & 1.230032 \\
\hline 0.00025 & $1.25 \mathrm{E}-06$ & 613 & 614 & 612 & 613 & 1.256117 \\
\hline 0.000275 & $1.38 \mathrm{E}-06$ & 604 & 602 & 601 & 602.3333 & 1.278362 \\
\hline 0.0003 & $1.5 \mathrm{E}-06$ & 588 & 590 & 590 & 589.3333 & 1.306561 \\
\hline
\end{tabular}

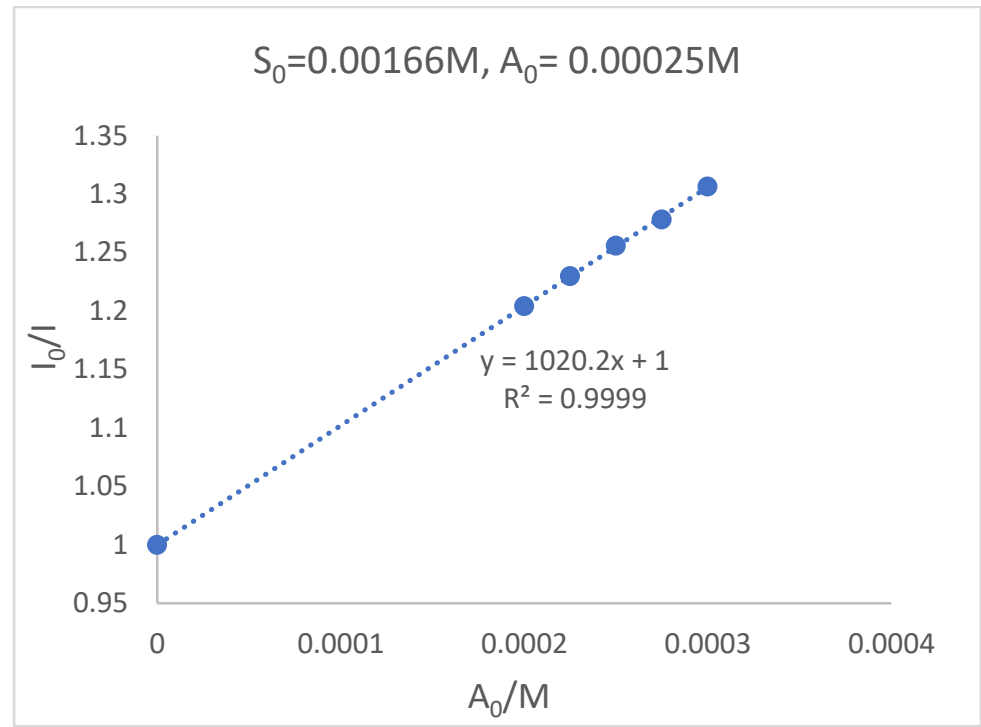

Figure S2.12.2. Stern-Volmer plot of luminescence data in Table S2.12.2.

$I_{0}$ was extrapolated from the five data points.

$$
\begin{gathered}
\frac{k_{S V}^{\prime \prime}+k_{o b s} \tau_{0} \mathrm{~S}_{0}}{1+k_{d S V}^{\prime} \mathrm{S}_{0}}=1020 \\
k_{S V}^{\prime \prime}+k_{o b s} \tau_{0} \mathrm{~S}_{0}=1020 \times(1+73 \times 0.00166)=1144 \\
148+0.005 k_{o b s} \tau_{0}=1144 \\
k_{o b s} \tau_{0}=600000
\end{gathered}
$$

Substitute $K_{\mathrm{A}}=44$ (from entry 11), $k_{\text {obs }} \tau_{0}=600000$ into equation a

$$
\begin{gathered}
0.00166+0.00025=\frac{0.00166 \times 0.00025 \times 600000}{k_{\text {PCET }} \tau_{0}}+\frac{k_{\text {PCET } \tau_{0}}^{\prime}}{600000}-\frac{1}{44} \\
k_{\text {PCET } \tau_{0}}^{\prime}=14800 \\
k_{\text {PCET average } \tau_{0}=15600}^{\prime} \text { a }
\end{gathered}
$$




$$
\begin{gathered}
k_{\text {PCET }} \tau_{0}=k_{\text {PCET }}^{\prime} \tau_{0}+k_{\text {SV }}^{\prime}=15600+148=15750 \\
\Delta G^{\circ}{ }_{\text {H-bond }}(\mathrm{kcal} / \mathrm{mol})=-\mathrm{RT} \ln K_{\mathrm{A}}=-2.24 \\
\ln \left[k_{\text {PCET }}\left(\mathrm{M}^{-1} \mathrm{~s}^{-1}\right)\right]=\ln \left[k_{\text {PCET }} / \tau_{0}\right]=22.65 \\
\tau_{0}=2.3 \mu \mathrm{s} \\
\text { error in } \Delta G^{\circ}{ }_{\text {-bond }}(\mathrm{kcal} / \mathrm{mol})=0.07 \\
\text { error in } \ln \left[k_{\mathrm{PCET}}\left(\mathrm{M}^{-1} \mathrm{~s}^{-1}\right)\right]=0.13
\end{gathered}
$$




\subsection{Entry 13.}

Thiol does not quench the luminescence of oxidant * Ir (III), therefore, the following equation is used:

$$
\frac{I_{0}}{I}=1+\left(k^{\prime}{ }_{S V}+k_{o b s} \tau_{0} S_{0}\right) A_{0}
$$

Table. S2.13.1. $S_{0}$ fixed at $0.0025 \mathrm{M}, \mathrm{A}_{0}$ varied from 0.0001 to $0.0003 \mathrm{M}$

\begin{tabular}{ccccccc}
\hline $\mathbf{A}_{0} / \mathbf{M}$ & $\mathrm{S}_{0} \mathbf{A}_{0} / \mathbf{M}^{2}$ & I/au 1 & I/au 2 & I/au 3 & I/au ave & $I_{0} / \mathbf{I}$ \\
0 & 0 & & & & & 1 \\
0.0001 & 0.00000025 & 108 & 107 & 107 & 107.3333 & 1.658385 \\
0.00015 & 0.000000375 & 88 & 88 & 88 & 88 & 2.022727 \\
0.0002 & 0.0000005 & 75 & 76 & 75 & 75.33333 & 2.362832 \\
0.00025 & 0.000000625 & 67 & 67 & 67 & 67 & 2.656716 \\
0.0003 & 0.00000075 & 59 & 59 & 60 & 59.33333 & 3 \\
\hline
\end{tabular}

$$
S_{0}=0.0025 \mathrm{M}, A_{0}=0.0002 M
$$

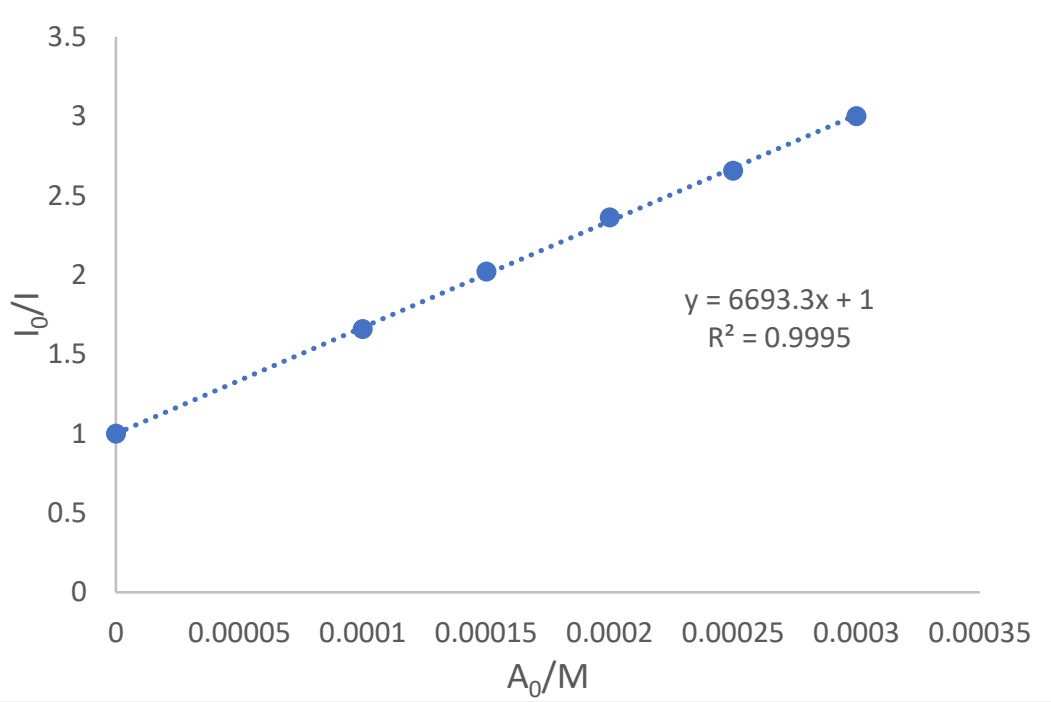

Figure S2.13.1. Stern-Volmer plot of luminescence data in Table S2.13.1.

$I_{0}$ was extrapolated from the five data points.

$$
\begin{gathered}
k^{\prime}{ }_{S V}+k_{o b s} \tau_{0} S_{0}=6693 \\
390+k_{o b s} \tau_{0} \times 0.0025=6693, k_{o b s} \tau_{0}=2521200
\end{gathered}
$$


Table. S2.13.2. $\mathrm{S}_{0}$ fixed at $0.0050 \mathrm{M}, \mathrm{A}_{0}$ varied from 0.0001 to $0.0003 \mathrm{M}$

\begin{tabular}{ccccccc}
\hline $\mathbf{A}_{0} / \mathbf{M}$ & $\mathbf{S}_{0} \mathbf{A}_{0} / \mathbf{M}^{2}$ & I/au 1 & I/au 2 & I/au 3 & I/au ave & $\mathbf{I}_{0} / \mathbf{I}$ \\
0 & 0 & & & & & 1 \\
0.0001 & 0.0000005 & 196 & 198 & 200 & 198 & 1.681818 \\
0.00015 & 0.00000075 & 164 & 164 & 163 & 163.6667 & 2.034623 \\
0.0002 & 0.000001 & 139 & 139 & 141 & 139.6667 & 2.384248 \\
0.00025 & 0.00000125 & 122 & 122 & 120 & 121.3333 & 2.744505 \\
0.0003 & 0.0000015 & 108 & 108 & 108 & 108 & 3.083333 \\
\hline
\end{tabular}

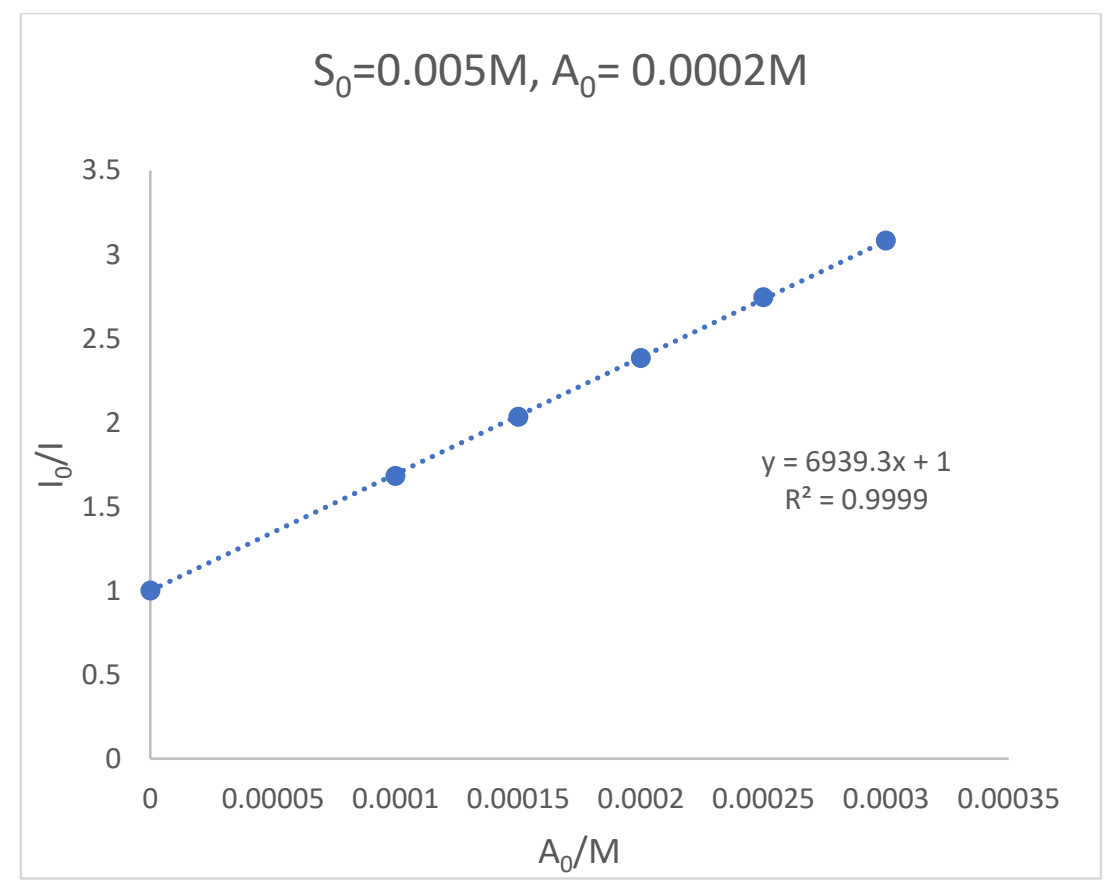

Figure S2.13.2. Stern-Volmer plot of luminescence data in Table S2.13.2.

$I_{0}$ was extrapolated from the five data points.

$$
\begin{gathered}
k^{\prime}{ }_{S V}+k_{o b s} \tau_{0} S_{0}=6939 \\
390+k_{o b s} \tau_{0} \times 0.0025=6939, k_{o b s} \tau_{0}=1309800
\end{gathered}
$$


Table. S2.13.3. $S_{0}$ fixed at $0.0080 \mathrm{M}, \mathrm{A}_{0}$ varied from 0.0001 to $0.0003 \mathrm{M}$

\begin{tabular}{ccccccc}
\hline $\mathbf{A}_{0} / \mathbf{M}$ & $\mathbf{S}_{\mathbf{0}} \mathbf{A}_{0} / \mathbf{M}^{2}$ & I/au 1 & I/au 2 & I/au 3 & I/au ave & $\mathbf{I}_{0} / \mathbf{I}$ \\
0 & 0 & & & & & 1 \\
0.0001 & 0.0000008 & 312 & 312 & 312 & 312 & 1.701923 \\
0.00015 & 0.0000012 & 260 & 258 & 258 & 258.6667 & 2.052835 \\
0.0002 & 0.0000016 & 217 & 217 & 217 & 217 & 2.447005 \\
0.00025 & 0.000002 & 190 & 193 & 193 & 192 & 2.765625 \\
0.0003 & 0.0000024 & 172 & 173 & 173 & 172.6667 & 3.07529 \\
\hline
\end{tabular}

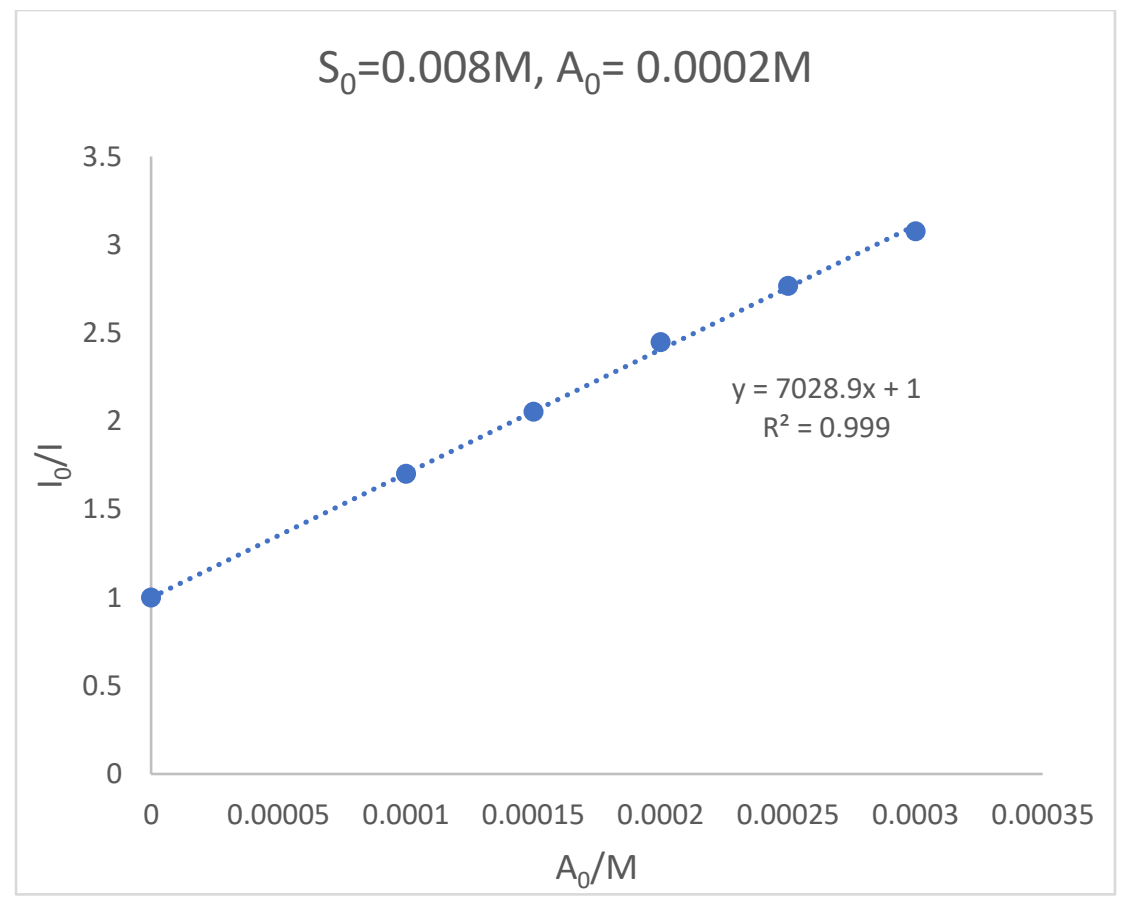

Figure S2.13.3. Stern-Volmer plot of luminescence data in Table S2.13.3.

$$
\begin{aligned}
& I_{0} \text { was extrapolated from the five data points. } \\
& \qquad k^{\prime}{ }_{S V}+k_{o b s} \tau_{0} S_{0}=7029 \\
& 390+k_{o b s} \tau_{0} \times 0.0025=7029, k_{o b s} \tau_{0}=829900
\end{aligned}
$$


Table. S2.13.4. $S_{0}$ fixed at $0.010 \mathrm{M}, \mathrm{A}_{0}$ varied from 0.0001 to $0.0003 \mathrm{M}$

\begin{tabular}{ccccccc}
\hline$A_{0} / \mathbf{M}$ & $\mathbf{S}_{0} A_{0} / M^{2}$ & I/au 1 & I/au 2 & I/au 3 & I/au ave & $I_{0} / \mathbf{I}$ \\
0 & 0 & & & & & 1 \\
0.00015 & 0.0000015 & 250 & 250 & 249 & 249.6667 & 2.05474 \\
0.0002 & 0.000002 & 212 & 212 & 212 & 212 & 2.419811 \\
0.00025 & 0.0000025 & 186 & 186 & 186 & 186 & 2.758065 \\
0.0003 & 0.000003 & 165 & 163 & 163 & 163.6667 & 3.13442 \\
0.00035 & 0.0000035 & 148 & 148 & 149 & 148.3333 & 3.458427 \\
\hline
\end{tabular}

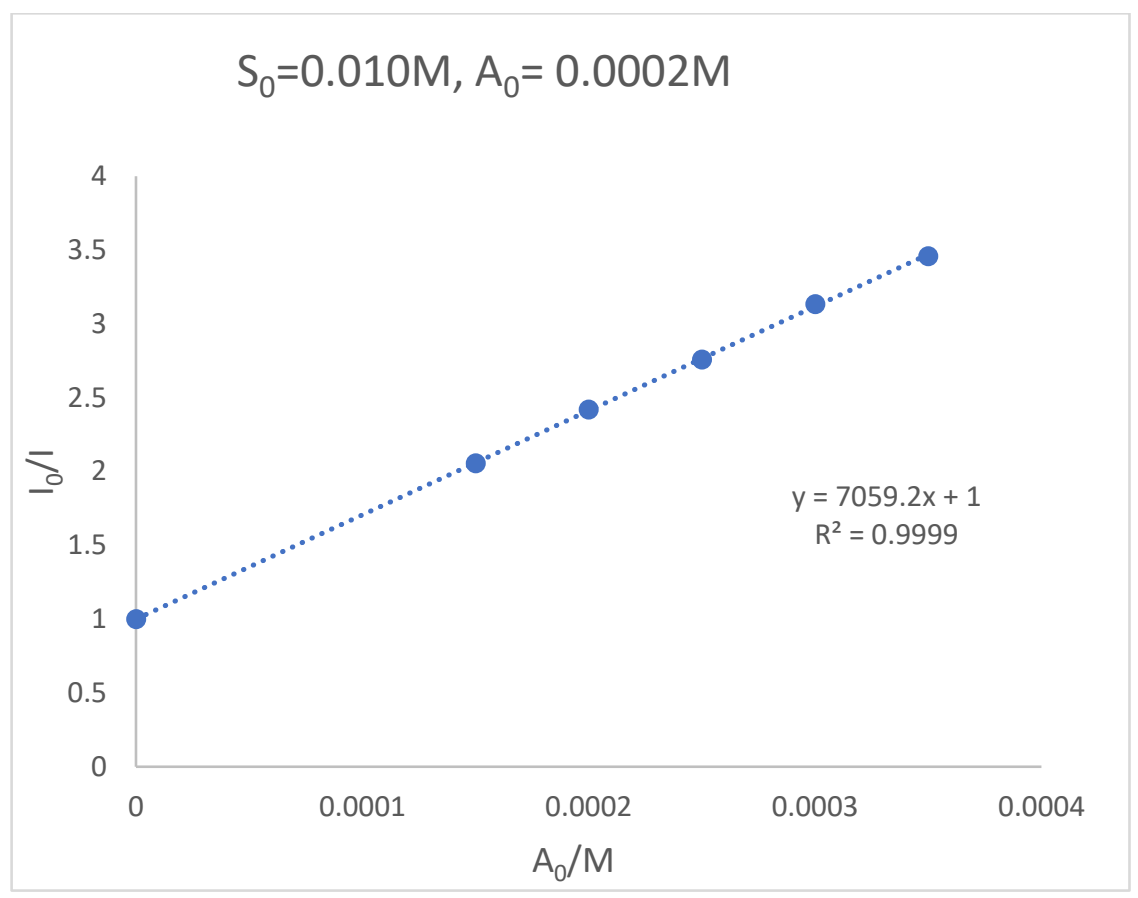

Figure S2.13.4. Stern-Volmer plot of luminescence data in Table S2.13.4.

$I_{0}$ was extrapolated from the five data points.

$$
k^{\prime}{ }_{S V}+k_{o b s} \tau_{0} S_{0}=7059
$$

$390+k_{o b s} \tau_{0} \times 0.0025=7059, k_{o b s} \tau_{0}=666900$ 
Table 2.13.5. Solutions to Key Equation a and Entry 13 in Table S2.

\begin{tabular}{|c|c|c|c|c|c|}
\hline & $S_{0}(M)$ & $A_{0}(M)$ & $S_{0} A_{0}\left(M^{2}\right)$ & $S_{0}+A_{0}(M)$ & $k_{o b s} \tau_{0}$ \\
\hline 1 & 0.0025 & 0.0002 & 5E-07 & 0.0027 & 2521200 \\
\hline 2 & 0.005 & 0.0002 & 0.000001 & 0.0052 & 1309800 \\
\hline 3 & 0.008 & 0.0002 & $1.6 \mathrm{E}-06$ & 0.0082 & 829875 \\
\hline \multirow[t]{15}{*}{4} & 0.01 & 0.0002 & 0.000002 & 0.0102 & 666900 \\
\hline & & & $K_{\mathrm{A}} / \mathrm{M}^{-1}$ & $k_{\text {РСETT }}^{\prime} / M^{-1}$ & \\
\hline & & $1 \& 2$ & 5532 & 6795 & \\
\hline & & $1 \& 3$ & 5574 & 6792 & \\
\hline & & $1 \& 4$ & 5582 & 6791 & \\
\hline & & $2 \& 3$ & 5685 & 6789 & \\
\hline & & $2 \& 4$ & 5684 & 6789 & \\
\hline & & $3 \& 4$ & 5680 & 6789 & \\
\hline & & average & 5600 & 6791 & \\
\hline & \multicolumn{4}{|c|}{$k_{\text {PCET }} \tau_{0}=k_{\text {РCET }}^{\prime} \tau_{0}+k_{\text {SV }}^{\prime}=6791+390=7180$} & \\
\hline & \multicolumn{4}{|c|}{$\Delta G^{\circ}{ }_{\text {H-bond }}(\mathrm{kcal} / \mathrm{mol})=-\mathrm{RT} \ln K_{\mathrm{A}}=-5.11$} & \\
\hline & \multicolumn{4}{|c|}{$\ln \left[k_{\text {PCET }}\left(\mathrm{M}^{-1} \mathrm{~S}^{-1}\right)\right]=\ln \left[k_{\text {PCET }} \mathrm{T}_{0} / \tau_{0}\right]=22.11$} & \\
\hline & \multicolumn{4}{|c|}{$\tau_{0}=1.8 \mu \mathrm{s}$} & \\
\hline & \multicolumn{4}{|c|}{ error in $\Delta G^{\circ}{ }_{\text {H-bond }}(\mathrm{kcal} / \mathrm{mol})=0.01$} & \\
\hline & & error $\mathrm{i}$ & {$\left[k_{\mathrm{PCET}}\left(\mathrm{M}^{-1} \mathrm{~S}^{-1}\right)\right.$} & ] $=0.01$ & \\
\hline
\end{tabular}




\subsection{Entry 14.}

Thiol does not quench the luminescence of oxidant * Ir (III), therefore, the following equation is used:

$$
\frac{I_{0}}{I}=1+\left(k^{\prime}{ }_{S V}+k_{o b s} \tau_{0} S_{0}\right) A_{0}
$$

Table. S2.14.1. $S_{0}$ fixed at $0.0080 \mathrm{M}, \mathrm{A}_{0}$ varied from 0.0001 to $0.0003 \mathrm{M}$

\begin{tabular}{ccccccc}
\hline $\mathbf{A}_{0} / \mathbf{M}$ & $\mathbf{S}_{0} \mathbf{A}_{0} / \mathbf{M}^{2}$ & I/au 1 & I/au 2 & I/au 3 & I/au ave & $I_{0} / \mathbf{I}$ \\
0 & 0 & & & & & 1 \\
0.0001 & 0.0000008 & 464 & 461 & 461 & 462 & 1.233766 \\
0.00015 & 0.0000012 & 424 & 424 & 424 & 424 & 1.34434 \\
0.0002 & 0.0000016 & 390 & 388 & 387 & 388.3333 & 1.467811 \\
0.00025 & 0.000002 & 359 & 358 & 359 & 358.6667 & 1.589219 \\
0.0003 & 0.0000024 & 334 & 334 & 335 & 334.3333 & 1.704885 \\
\hline
\end{tabular}

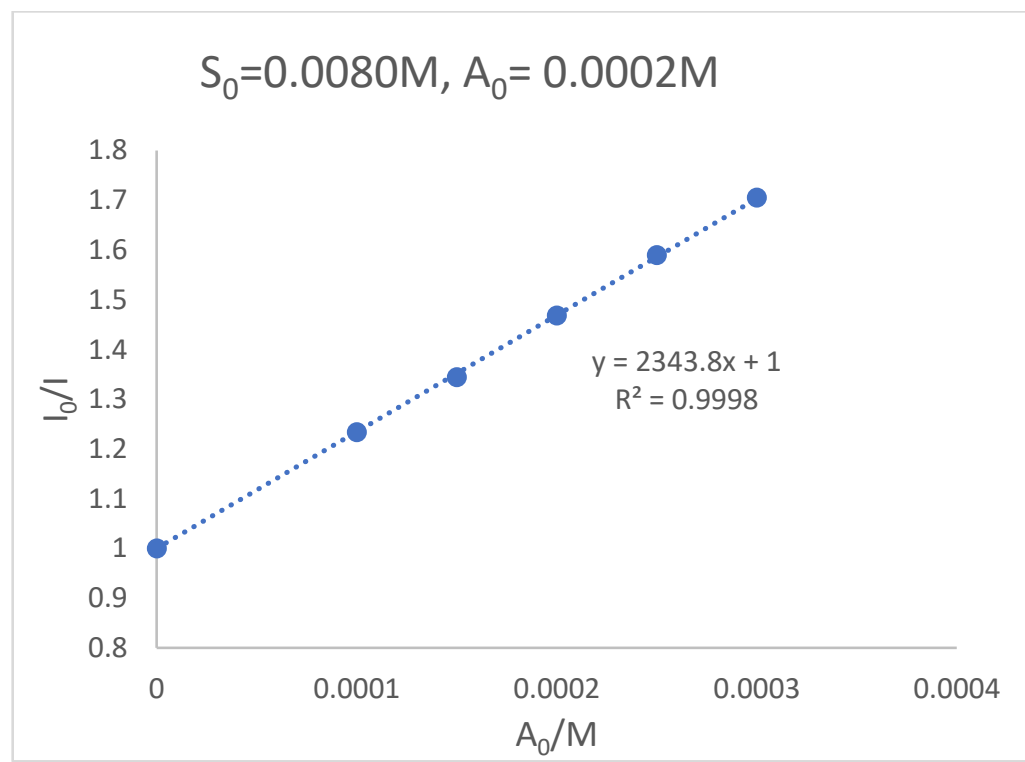

Figure S2.14.1. Stern-Volmer plot of luminescence data in Table S2.14.1.

$I_{0}$ was extrapolated from the five data points.

$$
\begin{gathered}
k^{\prime}{ }_{S V}+k_{o b s} \tau_{0} S_{0}=2344 \\
148+k_{o b s} \tau_{0} \times 0.0080=2344, k_{o b s} \tau_{0}=274500
\end{gathered}
$$

Substitute $K_{\mathrm{A}}=5600$ (from entry 7), $k_{o b s} \tau_{0}=274500$ into equation a 


$$
\begin{gathered}
0.008+0.0002=\frac{0.008 \times 0.0002 \times 274500}{k_{P C E T} \tau_{0}}+\frac{k_{P C E T}^{\prime} \tau_{0}}{274500}-\frac{1}{5600} \\
k_{\text {PCET } \tau_{0}=2246}^{\prime}
\end{gathered}
$$

Table. S2.14.2. $S_{0}$ fixed at $0.0098 \mathrm{M}, \mathrm{A}_{0}$ varied from 0.0001 to $0.0003 \mathrm{M}$

\begin{tabular}{ccccccc}
\hline $\mathbf{A}_{0} / \mathbf{M}$ & $\mathbf{S}_{\mathbf{0}} \mathbf{A}_{0} / \mathbf{M}^{2}$ & I/au 1 & I/au 2 & I/au 3 & I/au ave & $\mathbf{I}_{\mathbf{0}} / \mathbf{I}$ \\
0 & 0 & & & & & 1 \\
0.0001 & 0.00000098 & 547 & 548 & 549 & 548 & 1.206204 \\
0.00015 & 0.00000147 & 508 & 509 & 508 & 508.3333 & 1.300328 \\
0.0002 & 0.00000196 & 472 & 472 & 472 & 472 & 1.400424 \\
0.00025 & 0.00000245 & 445 & 445 & 444 & 444.6667 & 1.486507 \\
0.0003 & 0.00000294 & 418 & 415 & 415 & 416 & 1.588942 \\
\hline
\end{tabular}

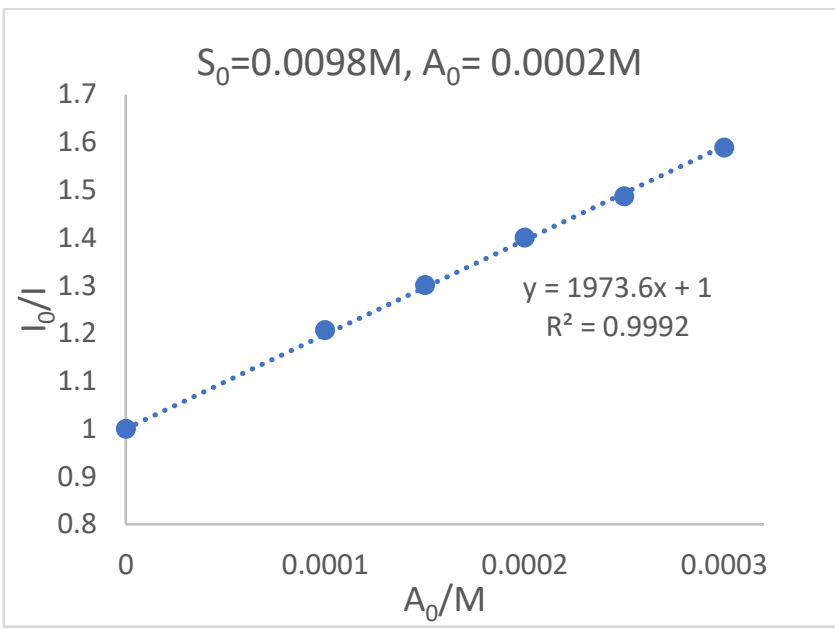

Figure S2.14.2. Stern-Volmer plot of luminescence data in Table S2.14.2.

$I_{0}$ was extrapolated from the five data points.

$$
\begin{gathered}
k^{\prime}{ }_{S V}+k_{o b s} \tau_{0} S_{0}=1974 \\
148+k_{o b s} \tau_{0} \times 0.0098=1974, k_{o b s} \tau_{0}=186330
\end{gathered}
$$

Substitute $K_{\mathrm{A}}=5600$ (from entry 7), $k_{o b s} \tau_{0}=186330$ into equation a

$$
\begin{gathered}
0.008+0.0002=\frac{0.008 \times 0.0002 \times 186330}{k_{P C E T} \tau_{0}}+\frac{k_{P C E T}^{\prime} \tau_{0}}{186330}-\frac{1}{5600} \\
k_{\text {PCET }}^{\prime} \tau_{0}=1860 \\
k_{\text {PCET }}^{\prime} \text { average } \tau_{0}=2053 \\
k_{\text {PCET }} \tau_{0}=k_{\text {PCET }}^{\prime} \tau_{0}+k_{\text {SV }}^{\prime}=2053+148=2200 \\
\Delta G^{\circ}{ }_{\text {-bond }}\left(\mathrm{Kcal}^{\prime} / \mathrm{mol}\right)=-\mathrm{RT} \ln K_{\mathrm{A}}=-5.11 \\
\ln \left[k_{\text {PCET }}\left(\mathrm{M}^{-1} \mathrm{~S}^{-1}\right)\right]=\ln \left[k_{\text {PCET }} / \tau_{0}\right]=20.72
\end{gathered}
$$




$$
\begin{gathered}
\tau_{0}=2.3 \mu \mathrm{s} \\
\text { error in } \Delta G^{\circ}{ }_{H-b o n d}(\mathrm{kcal} / \mathrm{mol})=0.01 \\
\text { error in } \ln \left[k_{\text {PCET }}\left(\mathrm{M}^{-1} \mathrm{~s}^{-1}\right)\right]=0.09
\end{gathered}
$$

\subsection{Entry 15.}

Thiol does not quench the luminescence of oxidant *Ir (III), therefore, the following equation is used:

$$
\frac{I_{0}}{I}=1+\left(k^{\prime}{ }_{S V}+k_{o b s} \tau_{0} S_{0}\right) A_{0}
$$

Table. S2.15.1. $S_{0}$ fixed at $0.0050 \mathrm{M}, \mathrm{A}_{0}$ varied from 0.0001 to $0.0003 \mathrm{M}$

\begin{tabular}{ccccccc}
\hline $\mathbf{A}_{0} / \mathbf{M}$ & $\mathrm{S}_{0} \mathrm{~A}_{0} / \mathrm{M}^{2}$ & I/au 1 & I/au 2 & I/au 3 & I/au ave & $\mathrm{I}_{0} / \mathbf{I}$ \\
0 & 0 & & & & & 1 \\
0.0001 & 0.0000005 & 283 & 285 & 285 & 284.3333 & 2.321219 \\
0.00015 & $7.5 \mathrm{E}-07$ & 222 & 221 & 221 & 221.3333 & 2.981928 \\
0.0002 & 0.000001 & 179 & 180 & 179 & 179.3333 & 3.680297 \\
0.00025 & $1.25 \mathrm{E}-06$ & 151 & 151 & 152 & 151.3333 & 4.361233 \\
0.0003 & 0.0000015 & 131 & 131 & 131 & 131 & 5.038168 \\
\hline
\end{tabular}

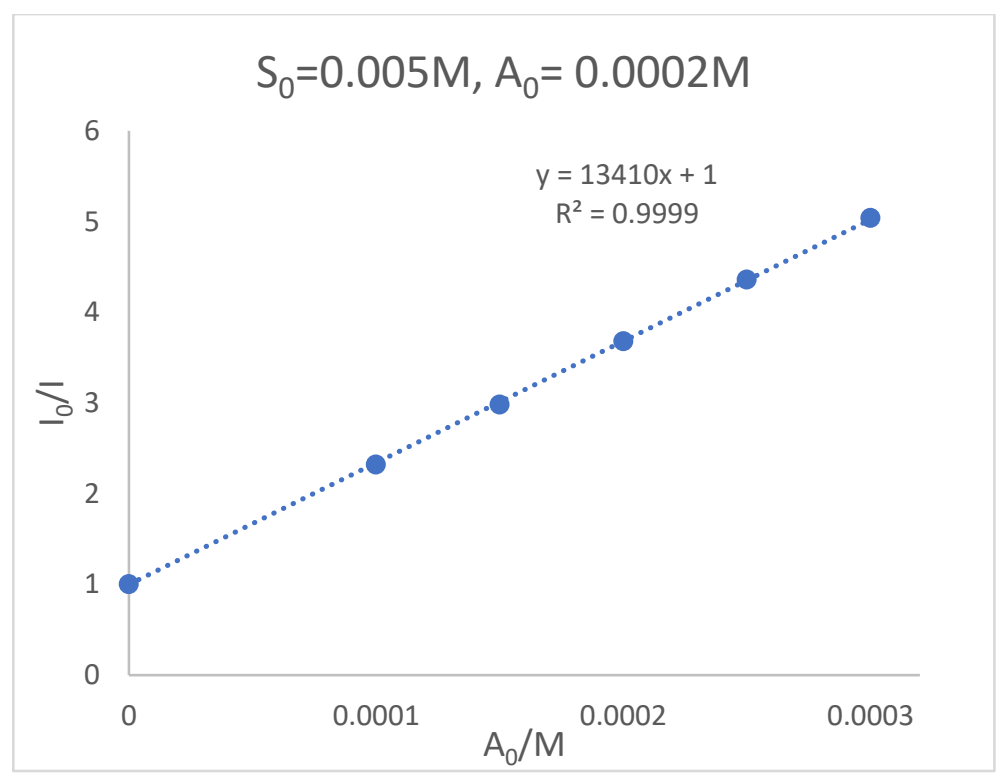

Figure S2.15.1. Stern-Volmer plot of luminescence data in Table S2.15.1. 
$I_{0}$ was extrapolated from the five data points.

$$
\begin{gathered}
k_{S V}^{\prime}+k_{o b s} \tau_{0} S_{0}=13410 \\
390+k_{o b s} \tau_{0} \times 0.005=13410, k_{o b s} \tau_{0}=2604000
\end{gathered}
$$

Table. S2.15.2. $S_{0}$ fixed at $0.0059 \mathrm{M}, \mathrm{A}_{0}$ varied from 0.00005 to $0.0003 \mathrm{M}$

\begin{tabular}{ccccccc}
\hline $\mathbf{A}_{0} / \mathbf{M}$ & $\mathbf{S}_{0} \mathbf{A}_{0} / \mathbf{M}^{2}$ & I/au 1 & I/au 2 & I/au 3 & I/au ave & $I_{0} / \mathbf{I}$ \\
0 & 0 & & & & & 1 \\
0.00005 & $2.94 \mathrm{E}-07$ & 728 & 730 & 729 & 729 & 1.668038 \\
0.0001 & $5.88 \mathrm{E}-07$ & 517 & 515 & 515 & 515.6667 & 2.358112 \\
0.00015 & $8.82 \mathrm{E}-07$ & 397 & 399 & 395 & 397 & 3.062972 \\
0.0002 & $1.176 \mathrm{E}-06$ & 329 & 327 & 330 & 328.6667 & 3.699797 \\
0.0003 & $1.764 \mathrm{E}-06$ & 239 & 239 & 238 & 238.6667 & 5.094972 \\
\hline
\end{tabular}

$$
S_{0}=0.0059 M, A_{0}=0.0002 M
$$

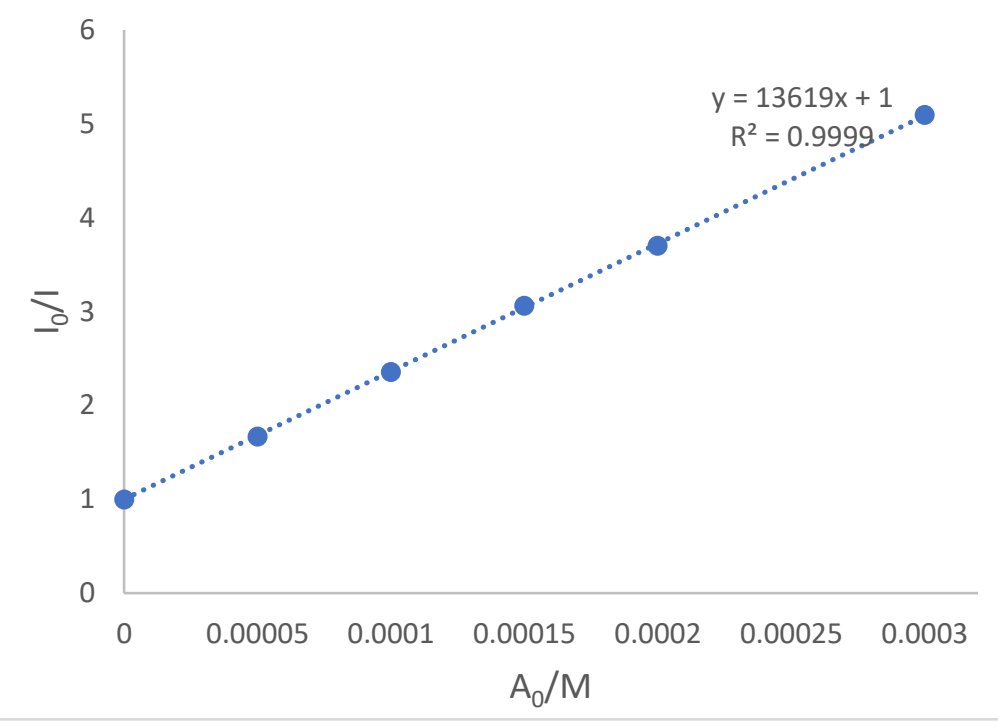

Figure S2.15.2. Stern-Volmer plot of luminescence data in Table S2.15.2.

$I_{0}$ was extrapolated from the five data points. 


$$
\begin{gathered}
k^{\prime}{ }_{S V}+k_{o b s} \tau_{0} S_{0}=13620 \\
390+k_{o b s} \tau_{0} \times 0.0059=13620, k_{o b s} \tau_{0}=2249800
\end{gathered}
$$

Table. S2.15.3. $S_{0}$ fixed at $0.0133 \mathrm{M}, \mathrm{A}_{0}$ varied from 0.00005 to $0.0003 \mathrm{M}$

\begin{tabular}{ccccccc}
\hline $\mathbf{A}_{0} / \mathbf{M}$ & $\mathbf{S}_{0} \mathbf{A}_{0} / \mathbf{M}^{2}$ & I/au 1 & I/au 2 & I/au 3 & I/au ave & $I_{0} / \mathbf{I}$ \\
0 & 0 & & & & & 1 \\
0.00005 & $6.65 E-07$ & 630 & 630 & 629 & 629.6667 & 1.72631 \\
0.0001 & $1.33 E-06$ & 448 & 447 & 447 & 447.3333 & 2.429955 \\
0.00015 & $1.995 E-06$ & 346 & 344 & 344 & 344.6667 & 3.153772 \\
0.0002 & $2.66 E-06$ & 283 & 282 & 284 & 283 & 3.840989 \\
0.0003 & $3.99 E-06$ & 204 & 206 & 206 & 205.3333 & 5.293831 \\
\hline
\end{tabular}

$$
S_{0}=0.0133 \mathrm{M}, A_{0}=0.0002 M
$$

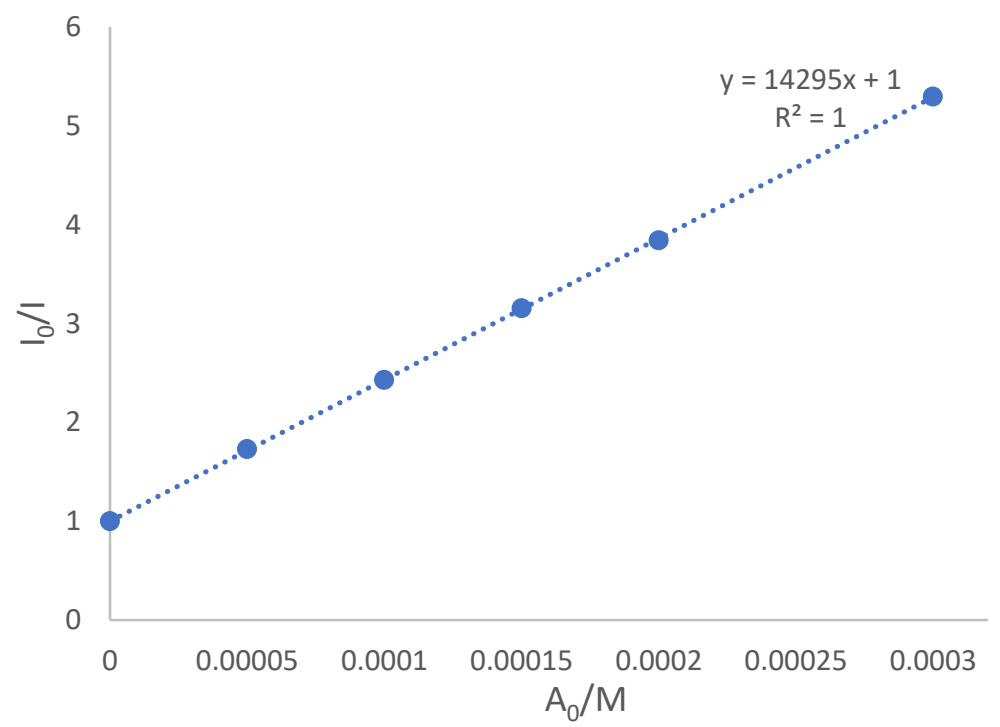

Figure S2.15.3. Stern-Volmer plot of luminescence data in Table S2.15.3.

$I_{0}$ was extrapolated from the five data points.

$$
\begin{gathered}
k_{S V}^{\prime}+k_{o b s} \tau_{0} S_{0}=14300 \\
390+k_{o b s} \tau_{0} \times 0.0133=14300, k_{o b s} \tau_{0}=1045500
\end{gathered}
$$


Table. S2.15.4. $S_{0}$ fixed at $0.0106 \mathrm{M}, \mathrm{A}_{0}$ varied from 0.00005 to $0.0003 \mathrm{M}$

\begin{tabular}{ccccccc}
\hline $\mathbf{A}_{0} / \mathbf{M}$ & $\mathbf{S}_{0} \mathbf{A}_{0} / \mathbf{M}^{2}$ & I/au 1 & I/au 2 & I/au 3 & I/au ave & $I_{0} / \mathbf{I}$ \\
0 & 0 & & & & & 1 \\
$5.71 \mathrm{E}-05$ & $6.057 \mathrm{E}-07$ & 398 & 402 & 398 & 399.3333 & 1.680301 \\
0.000114 & $1.211 \mathrm{E}-06$ & 252 & 252 & 251 & 251.6667 & 2.666225 \\
0.000171 & $1.817 \mathrm{E}-06$ & 191 & 191 & 191 & 191 & 3.513089 \\
0.000229 & $2.423 \mathrm{E}-06$ & 158 & 157 & 156 & 157 & 4.273885 \\
0.000286 & $3.029 \mathrm{E}-06$ & 135 & 134 & 134 & 134.3333 & 4.995037 \\
\hline
\end{tabular}

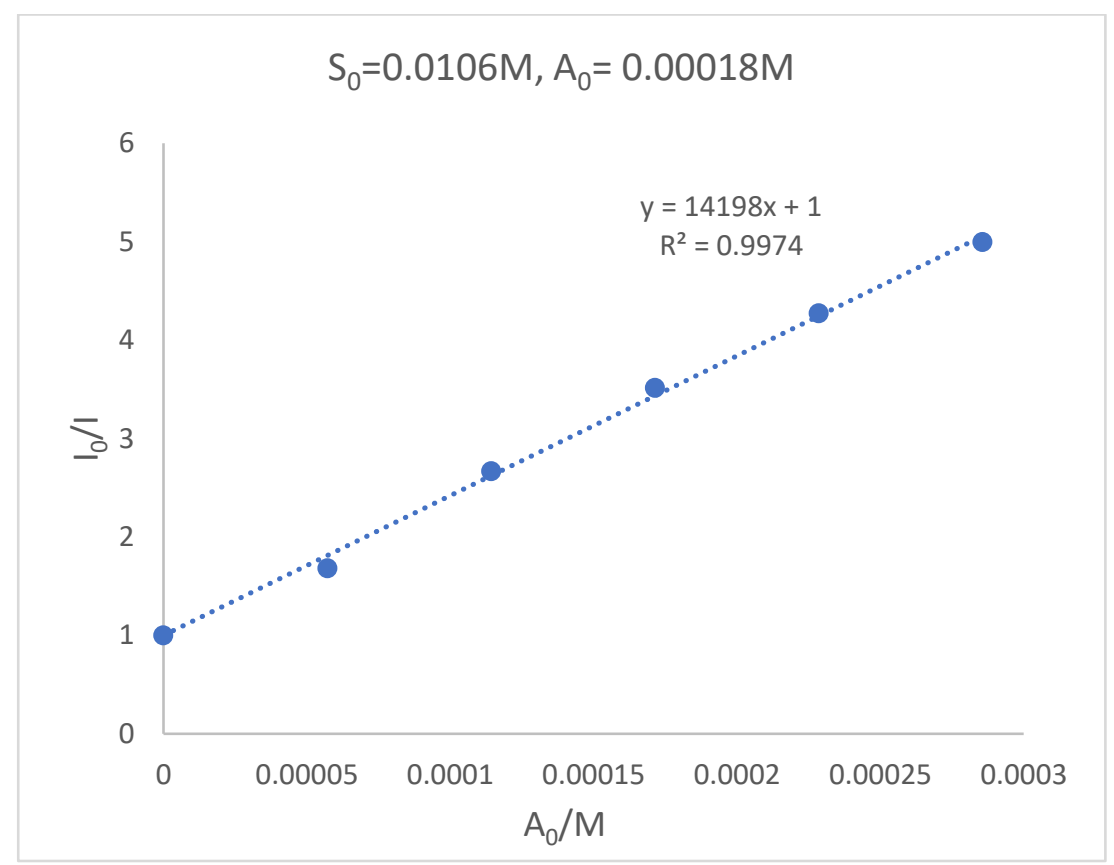

Figure S2.15.4. Stern-Volmer plot of luminescence data in Table S2.15.4.

$I_{0}$ was extrapolated from the five data points.

$$
\begin{gathered}
k^{\prime}{ }_{S V}+k_{o b s} \tau_{0} S_{0}=14200 \\
390+k_{o b s} \tau_{0} \times 0.0133=14200, k_{o b s} \tau_{0}=1302600
\end{gathered}
$$


Table 2.15.5. Solutions to Key Equation a and Entry 15 in Table S2.

\begin{tabular}{|c|c|c|c|c|c|}
\hline & $S_{0}(M)$ & $A_{0}(M)$ & $S_{0} A_{0}\left(M^{2}\right)$ & $S_{0}+A_{0}(M)$ & $k_{o b s} \tau_{0}$ \\
\hline 1 & 0.005 & 0.0002 & 0.000001 & 0.0052 & 2604000 \\
\hline 2 & 0.0059 & 0.0002 & $1.18 \mathrm{E}-06$ & 0.0061 & 2249800 \\
\hline 3 & 0.0133 & 0.0002 & $2.66 \mathrm{E}-06$ & 0.0135 & 1045500 \\
\hline 4 & 0.0106 & 0.00018 & $1.91 \mathrm{E}-06$ & 0.0108 & 1302600 \\
\hline & & & $K_{\mathrm{A}} / \mathrm{M}^{-1}$ & $k^{\prime}{ }_{\text {РСETТ }} / M^{-1}$ & \\
\hline & & $1 \& 2$ & 1814 & 14509 & \\
\hline & & $1 \& 3$ & 1853 & 14477 & \\
\hline & & 283 & 1854 & 14477 & \\
\hline & & $1 \& 4$ & 1692 & 14616 & \\
\hline & & $2 \& 4$ & 2980 & 14279 & \\
\hline & & $3 \& 4$ & 2700 & 14298 & \\
\hline & & average & 2150 & 14440 & \\
\hline \multicolumn{6}{|c|}{$k_{\mathrm{PCET}} \mathrm{T}_{0}=k_{\mathrm{PCET}}^{\prime} \mathrm{T}_{0}+k_{\mathrm{SV}}^{\prime}=14440+390=14830$} \\
\hline \multicolumn{6}{|c|}{$\Delta G^{\circ}{ }_{H-b o n d}(\mathrm{kcal} / \mathrm{mol})=-\mathrm{RT} \ln K_{\mathrm{A}}=-4.53$} \\
\hline \multicolumn{6}{|c|}{$\ln \left[k_{\text {PCET }}\left(\mathrm{M}^{-1} \mathrm{~S}^{-1}\right)\right]=\ln \left[k_{\text {PCET }} \mathrm{T}_{0} / \tau_{0}\right]=22.84$} \\
\hline \multicolumn{6}{|c|}{$\tau_{0}=1.8 \mu \mathrm{s}$} \\
\hline \multicolumn{6}{|c|}{ error in $\Delta G^{\circ}{ }_{\text {H-bond }}(\mathrm{kcal} / \mathrm{mol})=0.13$} \\
\hline
\end{tabular}




\subsection{Entry 16.}

Thiol does not quench the luminescence of oxidant *Ir (III), therefore, the following equation is used:

$$
\frac{I_{0}}{I}=1+\left(k^{\prime}{ }_{S V}+k_{o b s} \tau_{0} S_{0}\right) A_{0}
$$

Table. S2.16.1. $\mathrm{S}_{0}$ fixed at $0.0059 \mathrm{M}, \mathrm{A}_{0}$ varied from 0.00005 to $0.00025 \mathrm{M}$

\begin{tabular}{ccccccc}
\hline $\mathbf{A}_{0} / \mathbf{M}$ & $\mathbf{S}_{0} \mathbf{A}_{0} / \mathbf{M}^{2}$ & I/au 1 & I/au 2 & I/au 3 & I/au ave & $I_{0} / \mathbf{I}$ \\
0 & 0 & & & & & 1 \\
0.00005 & $2.94 \mathrm{E}-07$ & 622 & 619 & 622 & 621 & 1.231884 \\
0.0001 & $5.88 \mathrm{E}-07$ & 524 & 524 & 524 & 524 & 1.459924 \\
0.00015 & $8.82 \mathrm{E}-07$ & 465 & 465 & 465 & 465 & 1.645161 \\
0.0002 & $1.18 \mathrm{E}-06$ & 415 & 414 & 416 & 415 & 1.843373 \\
0.00025 & $1.47 \mathrm{E}-06$ & 373 & 373 & 375 & 373.6667 & 2.047279 \\
\hline
\end{tabular}

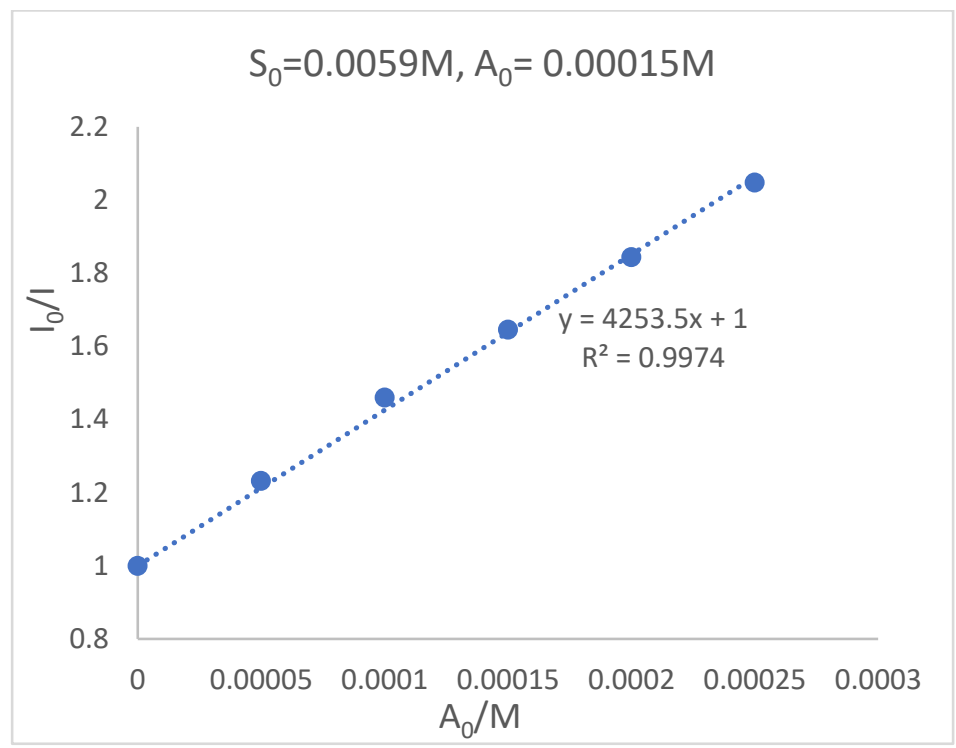

Figure S2.16.1. Stern-Volmer plot of luminescence data in Table S2.16.1.

$I_{0}$ was extrapolated from the five data points.

$$
\begin{gathered}
k^{\prime}{ }_{S V}+k_{o b s} \tau_{0} S_{0}=4254 \\
148+k_{o b s} \tau_{0} \times 0.0059=4254, k_{o b s} \tau_{0}=698300
\end{gathered}
$$

Substitute $K_{\mathrm{A}}=2150$ (from entry 7), $k_{o b s} \tau_{0}=698300$ into equation a

$$
\begin{gathered}
0.0059+0.00015=\frac{0.0059 \times 0.00015 \times 698300}{k_{P C E T} \tau_{0}}+\frac{k_{P C E T}^{\prime} \tau_{0}}{698300}-\frac{1}{2150} \\
k_{\text {PCET } \tau_{0}}^{\prime}=4550
\end{gathered}
$$


Table. S2.16.2. $S_{0}$ fixed at $0.0059 \mathrm{M}, \mathrm{A}_{0}$ varied from 0.00005 to $0.00025 \mathrm{M}$

\begin{tabular}{ccccccc}
\hline $\mathbf{A}_{0} / \mathbf{M}$ & $\mathbf{S}_{0} A_{0} / M^{2}$ & I/au 1 & I/au 2 & I/au 3 & I/au ave & $I_{0} / \mathbf{I}$ \\
0 & 0 & & & & & 1 \\
0.00005 & $6.65 \mathrm{E}-07$ & 522 & 522 & 522 & 522 & 1.226054 \\
0.0001 & $1.33 \mathrm{E}-06$ & 431 & 425 & 430 & 428.6667 & 1.493002 \\
0.00015 & $2 \mathrm{E}-06$ & 366 & 366 & 365 & 365.6667 & 1.750228 \\
0.0002 & $2.66 \mathrm{E}-06$ & 324 & 324 & 325 & 324.3333 & 1.973279 \\
0.00025 & $3.33 \mathrm{E}-06$ & 288 & 287 & 290 & 288.3333 & 2.219653 \\
\hline
\end{tabular}

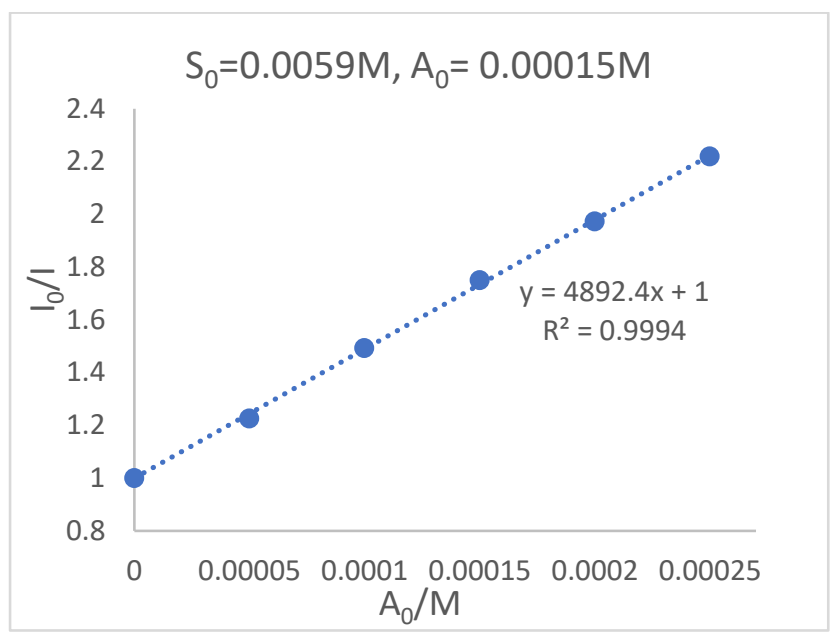

Figure S2.16.2. Stern-Volmer plot of luminescence data in Table S2.16.2.

$I_{0}$ was extrapolated from the five data points.

$$
\begin{gathered}
k^{\prime}{ }_{S V}+k_{o b s} \tau_{0} S_{0}=4892 \\
148+k_{o b s} \tau_{0} \times 0.0133=4892, k_{o b s} \tau_{0}=357000
\end{gathered}
$$

Substitute $K_{\mathrm{A}}=2150$ (from entry 7), $k_{o b s} \tau_{0}=357000$ into equation a

$$
\begin{aligned}
& 0.0133+0.00015=\frac{0.0133 \times 0.00015 \times 357000}{k_{P C E T} \tau_{0}}+\frac{k_{P C E T}^{\prime} \tau_{0}}{357000}-\frac{1}{2150} \\
& k_{\text {PCET }}^{\prime} \tau_{0}=4920 \\
& k_{\text {PCET }}^{\prime} \text { average } \tau_{0}=4735 \\
& k_{\text {PCET }} \mathrm{T}_{0}=k_{\text {PCET }}^{\prime} \mathrm{T}_{0}+k_{\mathrm{SV}}^{\prime}=4735+148=4880 \\
& \Delta G^{\circ}{ }_{\text {H-bond }}(\mathrm{kcal} / \mathrm{mol})=-\mathrm{RT} \ln K_{\mathrm{A}}=-4.53 \\
& \ln \left[k_{\text {PCET }}\left(M^{-1} \mathrm{~S}^{-1}\right)\right]=\ln \left[k_{\text {PCET }} \tau_{0} / \tau_{0}\right]=21.50 \\
& \tau_{0}=2.3 \mu \mathrm{s}
\end{aligned}
$$

error in $\Delta G^{\circ}{ }_{\text {-bond }}(\mathrm{kcal} / \mathrm{mol})=0.13$ error in $\ln \left[\mathrm{kPCET}_{\text {P }}\left(\mathrm{M}^{-1} \mathrm{~s}^{-1}\right)\right]=0.13$ 


\section{Energetics of MS-PCET}

$$
\begin{aligned}
& \text { Effective BDFE Equation }{ }^{2-4} \text { 'BDFE' }(\mathrm{kcal} / \mathrm{mol})=1.37 \mathrm{p} K_{\mathrm{a}}+23.06 \mathrm{E}_{0}+54.9 \\
& \Delta \mathrm{G}^{\circ}{ }_{\mathrm{H} \text {-bond }}=-\mathrm{R} \operatorname{Tn}\left(K_{\mathrm{A}}\right) \\
& \Delta \mathrm{G}^{\circ{ }_{\mathrm{PCET}}}=\mathrm{BDFE}_{\mathrm{N}-\mathrm{H} / \mathrm{S}-\mathrm{H}}+\Delta \mathrm{G}_{\mathrm{H}-\text { bond }}-{ }^{\mathrm{B}} \mathrm{BDFE}
\end{aligned}
$$

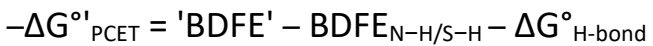

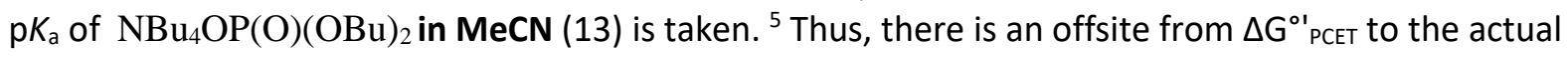
driving force in 1,2-dichloroethane.

\section{All BDFE $\mathrm{N}-\mathrm{H}_{\mathrm{H}}$ values are taken from one study. ${ }^{6}$}

The $\mathrm{BDFE}_{\mathrm{S-H}}$ values are taken from several studies. BDFE $\mathrm{S-H}_{-\mathrm{H}}$ of $\mathrm{T} 1$ and $\mathrm{T} 2$ are taken from the same study. ${ }^{7}$ BDFE $_{S-H}$ of T3 is taken from another source in which the same value of BDFE $_{S-H}$ for $T 1(79 \mathrm{kcal} / \mathrm{mol})$ is cited. ${ }^{8}$ To maximize the consistency, BDFE $_{S-H}$ of T4 is taken as the relative BDFE $_{S-H}$ between $\mathrm{T} 1$ and T4 (+9.37 kJ/mol, $+2.2 \mathrm{kcal} / \mathrm{mol}$ ) from a different study ${ }^{9}$ added to BDFE $\mathrm{S}_{-\mathrm{H}}$ of T1 in the former reference

\begin{tabular}{|c|c|c|c|c|c|c|c|}
\hline entry & $* \operatorname{Ir}(\mathrm{III})$ & $\begin{array}{c}\text { 'BDFE' } \\
\text { (kcal/mol) }\end{array}$ & $\begin{array}{l}\text { amide } \\
\text { /thiol }\end{array}$ & $\begin{array}{c}\mathrm{BDFE}_{\mathrm{N}-\mathrm{H} / \mathrm{S}-\mathrm{H}} \\
(\mathrm{kcal} / \mathrm{mol})\end{array}$ & $K_{\mathrm{A}}\left(\mathrm{M}^{-1}\right)$ & $\begin{array}{c}\Delta \mathrm{G}^{\circ} \mathrm{H} \text {-bond } \\
(\mathrm{kcal} / \mathrm{mol})\end{array}$ & $\begin{array}{l}\Delta \mathrm{G}^{\circ}{ }^{\prime} \mathrm{PCET} \\
(\mathrm{kcal} / \mathrm{mol})\end{array}$ \\
\hline I & $|r-|$ & 94.3 & $\mathrm{Al}$ & 98.9 & 1050 & -4.1 & 8.4 \\
\hline 2 & Ir-2 & 91.8 & Al & 98.9 & 1050 & -4.1 & 10.9 \\
\hline 3 & $|r-|$ & 94.3 & $\mathrm{~A} 2$ & 101.1 & 3550 & -4.8 & 11.3 \\
\hline 4 & Ir-2 & 91.8 & $\mathrm{~A} 2$ & 101.1 & 3550 & -4.8 & 13.8 \\
\hline 5 & $|r-|$ & 94.3 & A3 & 101.6 & 1390 & -4.3 & 11.2 \\
\hline 6 & Ir-2 & 91.8 & $\mathrm{~A} 3$ & 101.6 & 1390 & -4.3 & 13.7 \\
\hline 7 & $|r-|$ & 94.3 & A4 & 100.0 & 1500 & -4.3 & 9.7 \\
\hline 8 & Ir-2 & 91.8 & A4 & 100.0 & 1500 & -4.3 & 12.2 \\
\hline 9 & Ir-I & 94.3 & TI & 79.1 & 200 & -3.1 & -12.4 \\
\hline 10 & Ir-2 & 91.8 & TI & 79.1 & 200 & -3.1 & -9.8 \\
\hline 11 & $|r-|$ & 94.3 & $\mathrm{~T} 2$ & 76.9 & 44 & -2.2 & -15.6 \\
\hline 12 & Ir-2 & 91.8 & $\mathrm{~T} 2$ & 76.9 & 44 & -2.2 & -13.0 \\
\hline 13 & $|r-|$ & 94.3 & T3 & 84.0 & 5600 & -5.1 & -5.5 \\
\hline 14 & Ir-2 & 91.8 & T3 & 84.0 & 5600 & -5.1 & -3.0 \\
\hline 15 & Ir-I & 94.3 & $\mathrm{~T} 4$ & 81.3 & 2150 & -4.5 & -8.8 \\
\hline 16 & Ir-2 & 91.8 & $\mathrm{~T} 4$ & 81.3 & 2150 & -4.5 & -6.3 \\
\hline
\end{tabular}
(79.1 $\mathrm{kcal} / \mathrm{mol})$. i.e. $\mathrm{BDFE}_{\mathrm{S}-\mathrm{H}}$ of $\mathrm{T} 4=79.1+2.2=81.3 \mathrm{kcal} / \mathrm{mol}$

Table S3. Energetics of MS-PCET 


\section{Synthesis and Characterization of Substrates and Products}

All substrates were made according to the General Procedure B described in our previous work. ${ }^{10}$ The new compounds are characterized here. Characterization of other compounds can be found in our previous work. ${ }^{10}$

\section{N-(4-acetylphenyl)-5-methylhex-4-enamide}

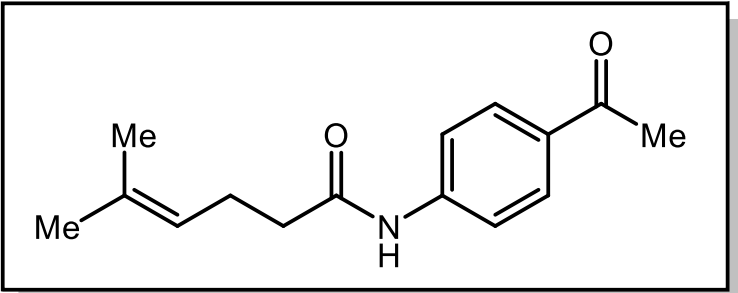

Synthesized using General Procedure B starting from 5methylhex-4-enoic acid and 4-Aminoacetophenone on a $2.76 \mathrm{mmol}$ scale with respect to the carboxylic acid. The product was purified by recrystallization from a mixture of hexanes and ethyl acetate to yield $372 \mathrm{mg}$ ( $50 \%$ yield) of the title compound as a white solid. IR (neat) 3258, 3188, 3110, 2966, 2934, 1679, 1666, 1591, $1526,1448,1403,1372,1350,1312,1262,1243,1172,1114,1074,1041,951,842,777,748 \mathrm{~cm}^{-1}$; ${ }^{1} \mathrm{H} \mathrm{NMR}$ (500 MHz, CDCl3) $\delta 7.93(\mathrm{~d}, J=8.4 \mathrm{~Hz}, 2 \mathrm{H}), 7.61(\mathrm{~d}, J=8.1 \mathrm{~Hz}, 2 \mathrm{H}), 5.20-5.12(\mathrm{~m}, 1 \mathrm{H}), 2.57(\mathrm{~s}, 3 \mathrm{H}), 2.44-$ $2.40(\mathrm{~m}, 4 \mathrm{H}), 1.71(\mathrm{~s}, 3 \mathrm{H}), 1.65(\mathrm{~s}, 3 \mathrm{H}) .{ }^{13} \mathrm{C} \mathrm{NMR}(126 \mathrm{MHz}, \mathrm{CDCl} 3) \delta 197.12,171.51,142.48,134.07,132.68$, $129.78,122.35,118.82,37.79,26.48,25.77,24.04,17.80$.

HRMS (ESI) exact mass calculated for $[\mathrm{M}+\mathrm{H}]^{+}\left(\mathrm{C}_{15} \mathrm{H}_{20} \mathrm{NO}_{2}\right)$ requires $\mathrm{m} / \mathrm{z} 246.1489$, found $\mathrm{m} / \mathrm{z} 246.1482$ difference $2.72 \mathrm{ppm}$.

\section{1-(4-acetylphenyl)-5-isopropylpyrrolidin-2-one}

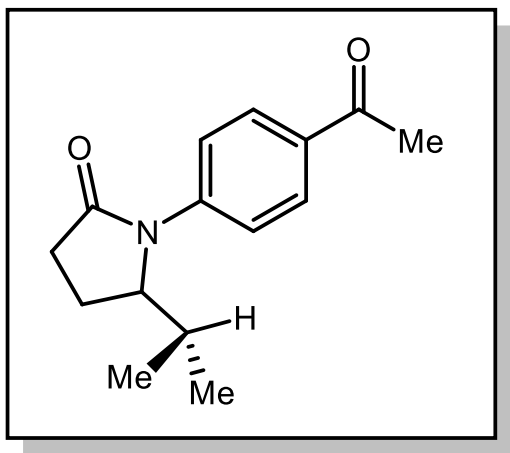

IR (neat) 2962, 2915, 1666, 1598, 1510, 1462, 1419, 1385, 1358, 1322, $1295,1262,1229,1182,1168,1121,1104,1073,1027,1012,954,850$, $737 \mathrm{~cm}^{-1} ;{ }^{1} \mathrm{H}$ NMR $(500 \mathrm{MHz}, \mathrm{CDCl} 3) \delta 7.98(\mathrm{~d}, J=8.4 \mathrm{~Hz}, 2 \mathrm{H}), 7.54(\mathrm{~d}$, $J=8.1 \mathrm{~Hz}, 2 \mathrm{H}), 4.36-4.31(\mathrm{~m}, 1 \mathrm{H}), 2.58(\mathrm{~s}, 3 \mathrm{H}), 2.66-2.50(\mathrm{~m}, 2 \mathrm{H}), 2.19-$ $2.01(\mathrm{~m}, 2 \mathrm{H}), 1.97-1.88(\mathrm{~m}, 1 \mathrm{H}), 0.92(\mathrm{~s}, 3 \mathrm{H}), 0.72(\mathrm{~s}, 3 \mathrm{H}) .{ }^{13} \mathrm{C} \mathrm{NMR}(126$ $\mathrm{MHz}, \mathrm{CDCl} 3) \delta$ 197.20, 174.85, 142.18, 133.94, 129.41, 123.20, 63.68, $31.89,28.47,26.68,18.57,17.65,14.41$

HRMS (ESI) exact mass calculated for $[\mathrm{M}+\mathrm{H}]^{+}\left(\mathrm{C}_{15} \mathrm{H}_{20} \mathrm{NO}_{2}\right)$ requires $\mathrm{m} / \mathrm{z}$ 246.1489 , found $\mathrm{m} / \mathrm{z} 246.1482$ difference $2.72 \mathrm{ppm}$. 

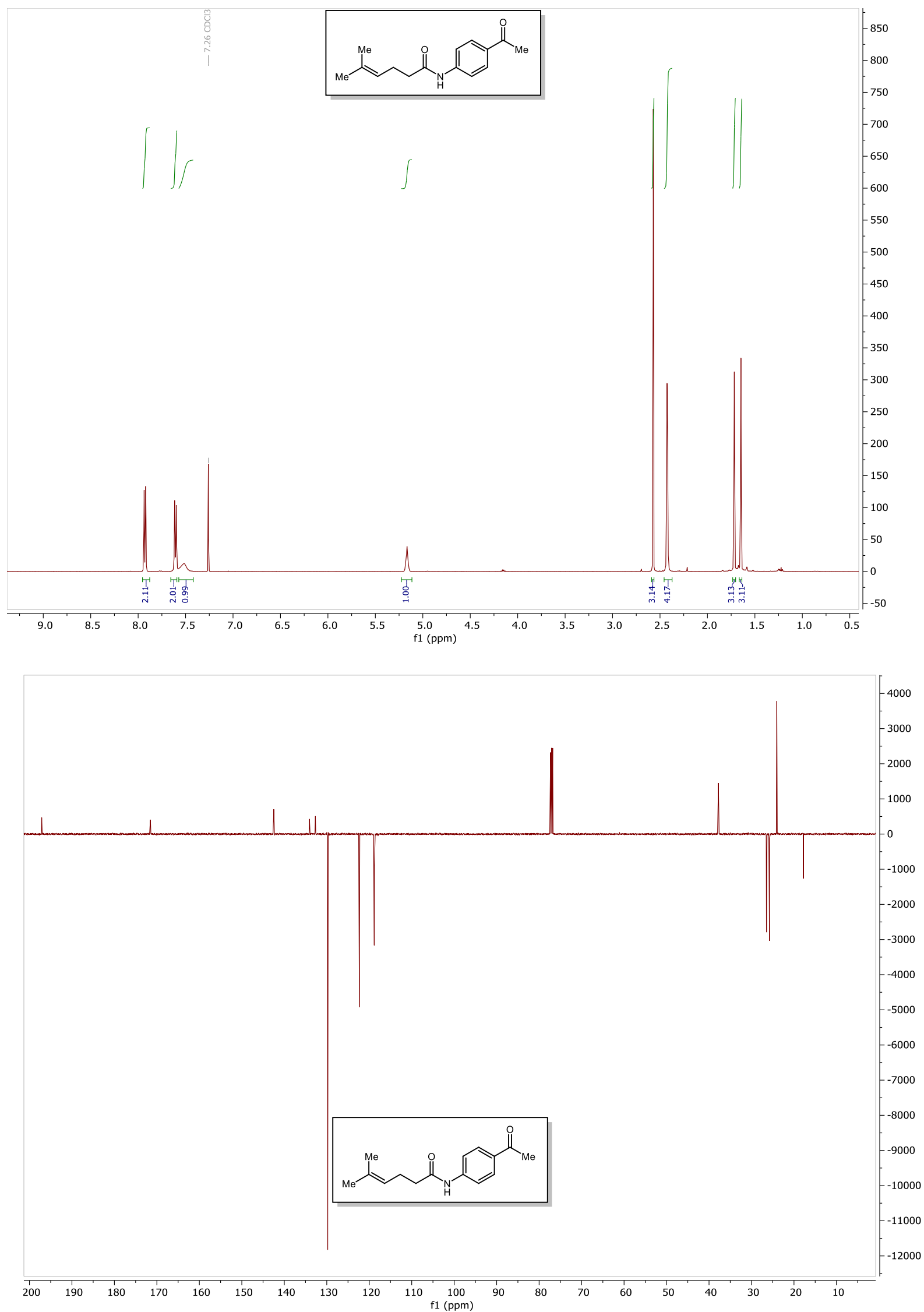

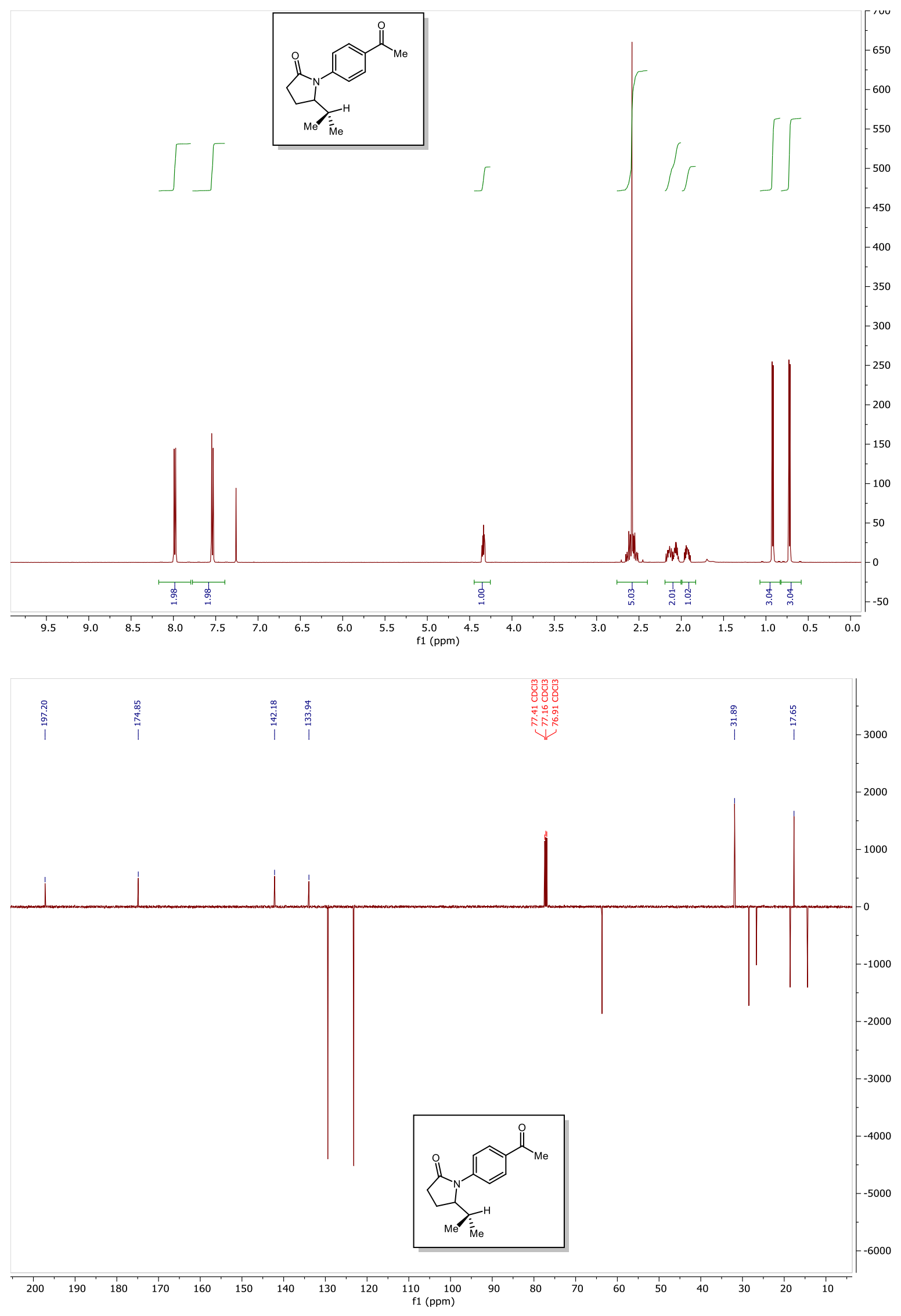


\section{Competition Stern-Volmer Experiments as Verification for MS-PCET Selectivity}

\subsection{Qualitative Competition-Quenching Experiments}

In our previous work, we studied the luminescence quenching of Ir-1 by the mixture of amide $\mathbf{A} \mathbf{1}$, thiol T1, and the phosphate base. ${ }^{10}$ When luminescence is observed as a function of the thiol concentration in the presence of base and amide, the observed quenching / does not vary relative to the background quenching by the amide adduct incorporated into the $I_{0}$ luminescence. These results indicate thiol adduct quenching is inefficient relative to amide adduct quenching. When the opposite experiment is run, i.e. when [amide] is varied and [thiol] is held constant in excess, the observed quenching / demonstrates a clean first-order dependence on the concentration of acetanilide relative to the background quenching $I_{o}$. (Figure S5.1.1) Taken together, these experiments demonstrate that PCET to the amide is kinetically preferred over PCET to the thiophenol component.

Orange

\begin{tabular}{|c|c|}
\hline Species & Concentration $(\mathrm{mM})$ \\
\hline$\left[\operatorname{Ir}(\mathrm{dF}(\mathrm{CF} 3) \mathrm{ppy})_{2}(\mathrm{bpy})\right]\left(\mathrm{PF}_{6}\right)$ & 0.01 \\
$\mathrm{Bu}_{4} \mathrm{~N}^{+}(\mathrm{BuO})_{2} \mathrm{PO}_{2}^{-}$ & 0.20 \\
Thiophenol & 1.00 \\
Acetanilide & Varied \\
\hline
\end{tabular}

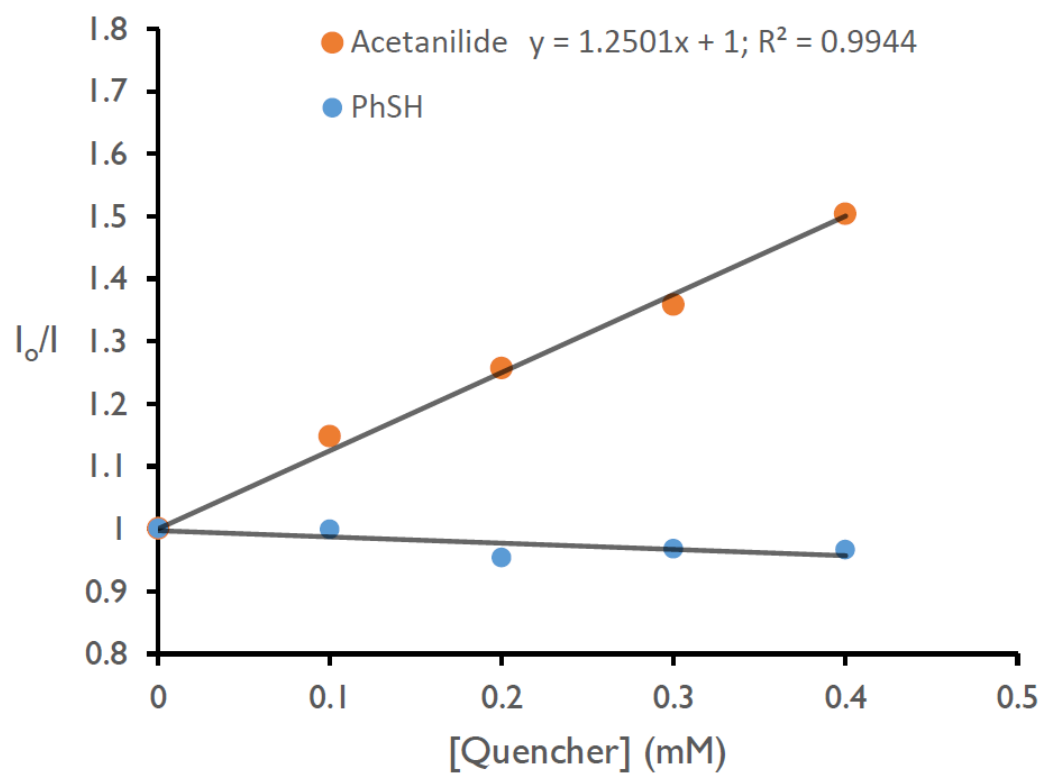

Figure S5.1.1. Competition Stern-Volmer experiment with the combination of the reagents corresponds to an effective hydroamidation reaction 
In this work, we extend the similar experiment to a different combination of amide (A2), thiol (T3), and oxidant (Ir-2) that correspond to an ineffective hydroamidation reaction (Table 2 entry 18 in the main text). The result here is the exact opposite. When luminescence is observed as a function of the amide concentration in the presence of base and thiol, the observed quenching I does not diminish relative to the background quenching by the thiol adduct incorporated into the $I_{o}$ luminescence. When the opposite experiment is run, i.e. when [thiol] is varied and [amide] is held constant, the observed quenching $I$ demonstrates a clean first-order dependence on the concentration of thiol relative to the background quenching $I_{o}$. (Figure S5.1.2) These experiments demonstrate that PCET to the thiol is kinetically preferred over PCET to the amide component.

Blue
\begin{tabular}{cc} 
Species & Concentration (M) \\
Oxidant Ir-2 & 0.00001 \\
$\mathrm{Bu}_{4} \mathrm{~N}^{+}(\mathrm{BuO})_{2} \mathrm{PO}_{2}{ }^{-}$ & 0.00015 \\
Thiol T3 & 0.001 \\
Amide A2 & Varied \\
\hline
\end{tabular}

\begin{tabular}{lc} 
Orange \\
Species & Concentration (M) \\
\hline Oxidant Ir-2 & 0.00001 \\
$\mathrm{Bu}_{4} \mathrm{~N}^{+}(\mathrm{BuO})_{2} \mathrm{PO}_{2}^{-}$ & 0.0001 \\
Thiol T3 & Varied \\
Amide A2 & 0.001 \\
\hline
\end{tabular}

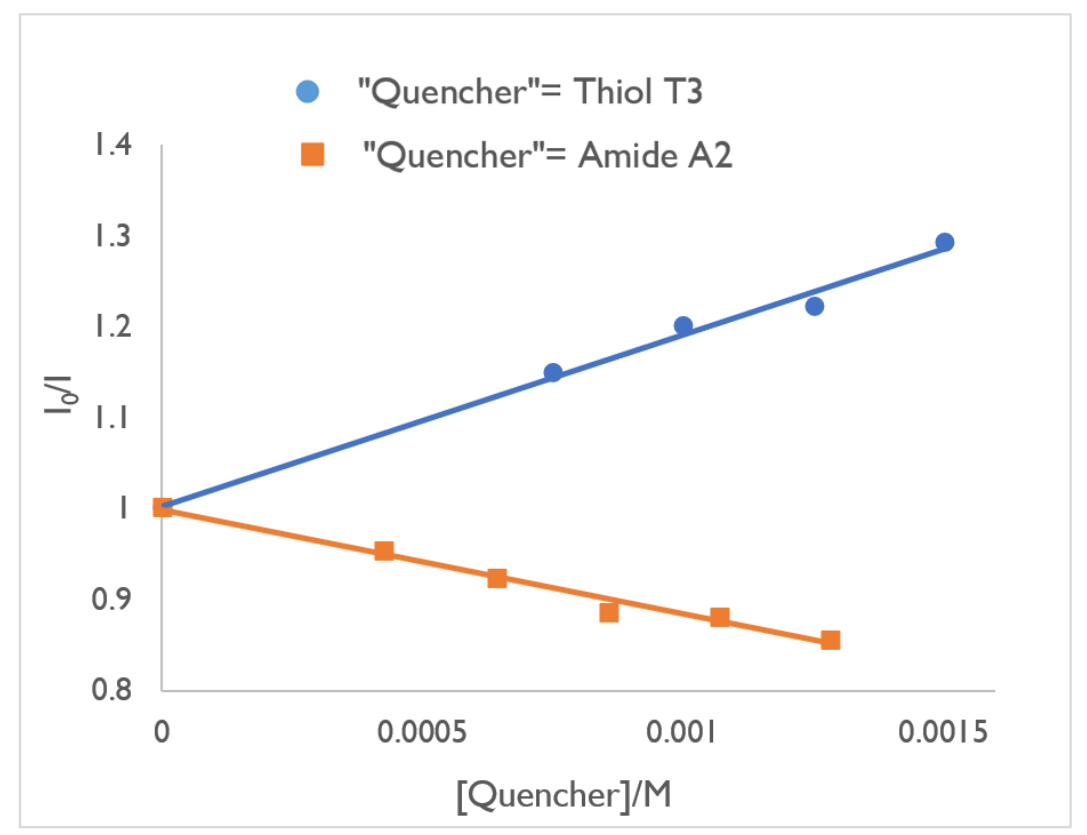

Figure S5.1.2. Competition Stern-Volmer experiment with the combination of the reagents corresponds to an ineffective hydroamidation reaction 


\subsection{Quantitative Competition-Quenching Experiments}

Equal concentration $(0.001 \mathrm{M})$ of amide $\mathbf{A} \mathbf{2}$ and thiol $\mathbf{T} \mathbf{1}$, were mixed, with varying concentration of the phosphate base $\mathrm{NBu}_{4} \mathrm{OP}(\mathrm{O})(\mathrm{OBu})_{2}$. to determine the quenching constant of the luminescence of oxidant Ir-1. Kinetic data of PCET quenching were determined experimentally and were compared with the predicted value according to the $k_{\text {PCET }}$ and $K_{\mathrm{A}}$ of $\mathbf{A} 2$ and $\mathbf{T} 1$ determined above. In all experiments, the solution was irradiated at $380 \mathrm{~nm}$ and fluorescence was measured at $496 \mathrm{~nm}$.

\subsubsection{Experimental Quenching Constant}

Table S5.2.1.1 Luminescence quenching data with [amide] and [thiol] fixed at 0.001M, [base] varied from 0.000075 to $0.000125 \mathrm{M}$.

\begin{tabular}{cccccc}
\hline $\begin{array}{c}\text { [base]/M } \\
0\end{array}$ & I/au 1 & I/au 2 & I/au 3 & I/au ave & Io/I \\
0.000075 & 400 & 401 & 402 & 401 & 1.15212 \\
0.0000875 & 392 & 392 & 392 & 392 & 1.178571 \\
0.0001 & 385 & 383 & 385 & 384.3333 & 1.202082 \\
0.0001125 & 377 & 376 & 377 & 376.6667 & 1.226549 \\
0.000125 & 368 & 369 & 370 & 369 & 1.252033 \\
\hline
\end{tabular}

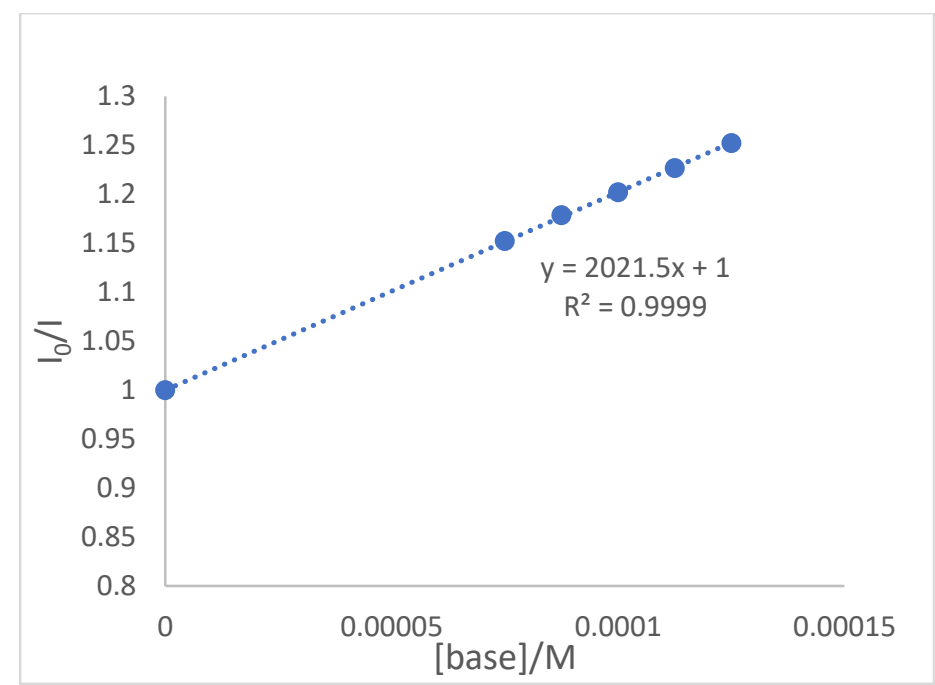

Figure S5.2.1.1 Stern-Volmer plot of luminescence data in Table S5.2.1.1

$I_{0}$ was extrapolated from the five data points.

$$
\begin{gathered}
\mathrm{I}_{0} / \mathrm{I}=1+k_{\text {obs exp }} \tau_{0}([\text { amide }]+[\text { thiol }])[\text { base }] \\
k_{\text {obs exp }} \tau_{0}([\text { amide }]+[\text { thiol }])=2021
\end{gathered}
$$


Table S5.2.1.2. Repeat. Luminescence quenching data with [amide] and [thiol] fixed at $0.001 \mathrm{M}$, [base] varied from 0.000075 to $0.000125 \mathrm{M}$.

\begin{tabular}{cccccc}
\hline $\begin{array}{c}\text { [base]/M } \\
0\end{array}$ & I/au 1 & I/au 2 & I/au 3 & I/au ave & $I_{0} / \mathbf{I}$ \\
0.000075 & 187.5 & 187.3 & 187.6 & 187.4667 & 1.151671 \\
0.0000875 & 183.8 & 183.5 & 183.6 & 183.6333 & 1.175712 \\
0.0001 & 179.2 & 179.8 & 180 & 179.6667 & 1.20167 \\
0.0001125 & 176.2 & 175.7 & 175.7 & 175.8667 & 1.227635 \\
0.000125 & 173 & 173 & 173.1 & 173.0333 & 1.247736 \\
\hline
\end{tabular}

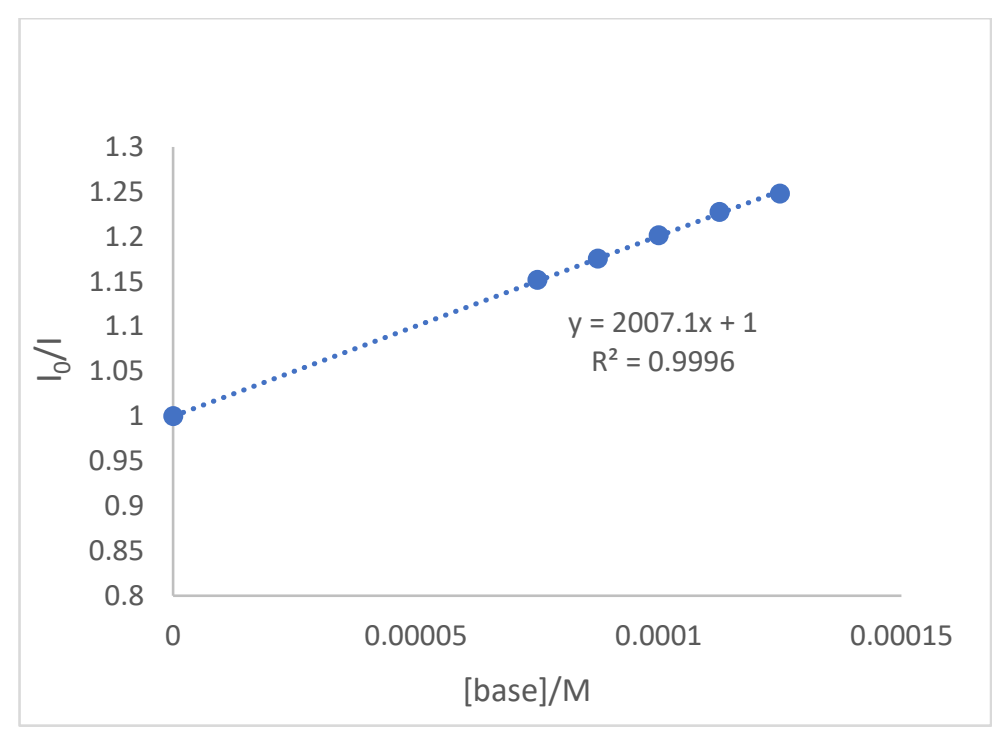

Figure S5.2.1.2. Stern-Volmer plot of luminescence data in Table S5.2.1.2.

$$
\begin{gathered}
I_{0} \text { was extrapolated from the five data points. } \\
I_{0} / I=1+k_{\text {obs } \exp } \tau_{0}([\text { amide }]+[\text { thiol }])[\text { base }] \\
k_{\text {obs } \exp } \tau_{0}([\text { amide }]+[\text { thiol }])=2007
\end{gathered}
$$




\subsubsection{Predicted Quenching Constant}

In the mixture of thiol, amide and base, (assume that thiol-base and amide-base are the only two intermolecular interactions between solutes) the following three equilibria are co-established:<smiles>Sc1ccccc1</smiles><smiles>CC(=O)Nc1ccc(C#N)cc1</smiles><smiles>O=P(O)(O)OCCCCCO</smiles><smiles></smiles><smiles>O=P([O-])([O-])[O-]</smiles><smiles>Sc1ccccc1</smiles><smiles>O=P([O-])([O-])O</smiles>

$S-x$

$N$
$N-y$

$P$

0

0

$P-x-y$

$\mathbf{x}$

y

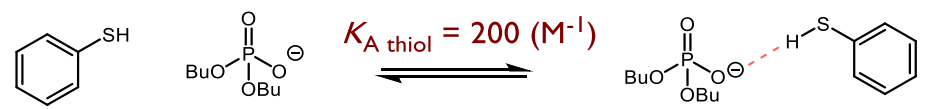

$\mathrm{x}$ and $\mathrm{y}$ are concentrations of the two pre-PCET hydrogen-bonded adduct for thiol T1 and amide $\mathbf{A} \mathbf{2}$ respectively. These two concentrations satisfy the simultaneous equations

$$
\begin{aligned}
& K_{\text {A thiol }}=\frac{x}{(S-x)(P-x-y)}=200\left(K_{\text {A thiol }} \text { from Table S2 entry } 9\right) \\
& K_{\text {A amide }}=\frac{y}{(N-y)(P-x-y)}=3550\left(K_{\text {A amide }} \text { from Table S2 entry } 3\right)
\end{aligned}
$$

Experimentally, the plot $\frac{I_{0}}{I}=1+k_{S V}(S+N) P$, is adopted and the gradient $k_{S V}(S+N)$ is taken as the "experimental $k_{\text {obs }} \tau_{0}$ ". Here "experimental $k_{\text {obs }} \tau_{0} "=1 / 2 \times(2021+2007)=2014$ 
The predicted plot is $\frac{I_{0}}{I}=1+k_{\text {PCET thiol }} \tau_{0} x+k_{\text {PCET amide }} \tau_{0} y . \quad k_{\text {PCET thiol }}$ and $k_{\text {PCET amide }}$ are the PCET rate constant pre-determined in Table S2 entry 9 and entry 3 respectively.

Thus, for any given initial concentrations of thiol, amide and base, the concentration of each of the two pre-PCET hydrogen-bonded adducts can be estimated.

To parallel the experimental " $k_{\mathrm{obs}} \tau_{0} ", \frac{I_{0}}{I}=1+\frac{k_{\text {PCET thiol }} \tau_{0}[x]+k_{P C E T ~ a m i d e} \tau_{0}[y]}{P_{\text {average }}}[$ base $]$ is adopted, and the "predicted " $k_{\mathrm{obs}} \tau_{0}$ " is $\left(\frac{k_{P C E T \text { thiol }} \tau_{0} x+k_{\text {PCET amide }} \tau_{0} y}{[\text { base }]_{\text {average }}}\right)$. In the experiments described in Section 5.2.1, [base $]_{0}$ is varied as $0.000075,0.0000875,0.00010,0.0001125,0.000125 \mathrm{M},[\text { base }]_{\text {average }}$ is then taken as $0.0001 \mathrm{M}$.

In Section 5.2.1, $\mathrm{S}=0.001 \mathrm{M}, \mathrm{N}=0.001 \mathrm{M}, \mathrm{P}=0.0001 \mathrm{M}$, [base $]_{\text {average }}=\mathrm{P}=0.0001 \mathrm{M}$.

$$
\begin{gathered}
\frac{y}{(0.001-y)(0.0001-x-y)}=3550 \\
\frac{x}{(0.001-x)(0.0001-x-y)}=200 \\
\mathrm{y}=7.33 \times 10^{-5} \mathrm{M}, \mathrm{x}=4.44 \times 10^{-6} \mathrm{M} \\
k_{\text {PCET amide }}=9.3 \times 10^{8} \mathrm{M}^{-1} \mathrm{~s}^{-1}, k_{\mathrm{PCET} \text { thiol }}=9.5 \times 10^{9} \mathrm{M}^{-1} \mathrm{~s}^{-1} \\
\text { "predicted } k_{\text {obs }} \tau_{0} "=\left(\frac{k_{\text {PCET thiol }} \tau_{0} x+k_{\text {PCET amide }} \tau_{0} y}{0.0001}\right)=2008
\end{gathered}
$$

"predicted $k_{\mathrm{obs}} \tau_{0}$ " and "experimental $k_{\mathrm{obs}} \tau_{0}$ " are in high agreement.

To appreciate the significance of such agreement, if the $K_{\mathrm{A}}$ and $k_{\mathrm{PCET}}$ for amide $\mathbf{A} \mathbf{3}$ instead of $\mathbf{A} \mathbf{2}$ is substituted instead:

$$
\begin{aligned}
& \frac{y}{(0.001-y)(0.0001-x-y)}=1400 \\
& \frac{x}{(0.001-x)(0.0001-x-y)}=200
\end{aligned}
$$




$$
\begin{gathered}
\mathrm{y}=5.25 \times 10^{-5} \mathrm{M}, \mathrm{x}=7.86 \times 10^{-6} \mathrm{M} \\
k_{\mathrm{PCET} \text { amide }}=1.1 \times 10^{9} \mathrm{M}^{-1} \mathrm{~s}^{-1}, k_{\mathrm{PCET} \text { thiol }}=9.5 \times 10^{9} \mathrm{M}^{-1} \mathrm{~s}^{-1} \\
\text { "predicted } k_{\mathrm{obs}} \tau_{0} "=\left(\frac{k_{\text {PCET thiol }} \tau_{0} x+k_{\text {PCET amide }} \tau_{0} y}{0.0001}\right)=2410
\end{gathered}
$$

Similarly, if the $K_{\mathrm{A}}$ and $k_{\mathrm{PCET}}$ for thiol $\mathbf{T} 2$ Instead of $\mathbf{T} 1$ is substituted instead:

$$
\begin{gathered}
\frac{y}{(0.001-y)(0.0001-x-y)}=3550 \\
\frac{x}{(0.001-x)(0.0001-x-y)}=44 \\
\mathrm{y}=7.59 \times 10^{-5} \mathrm{M}, \mathrm{x}=1.02 \times 10^{-6} \mathrm{M} \\
k_{\text {PCET amide }}=9.3 \times 10^{8} \mathrm{M}^{-1} \mathrm{~s}^{-1}, k_{\text {PCET thiol }}=1.0 \times 10^{10} \mathrm{M}^{-1} \mathrm{~s}^{-1} \\
\text { "predicted } k_{\mathrm{obs}} \tau_{0} "=\left(\frac{k_{\text {PCET thiol }} \tau_{0} x+k_{\text {PCET amide }} \tau_{0} y}{0.0001}\right)=1470
\end{gathered}
$$

Both hypothetical "predicted $k_{\mathrm{obs}} \tau_{0}$ " values deviate significantly from the "experimental $k_{\mathrm{obs}} \tau_{0}$ " 


\section{Calculation of MS-PCET Selectivity Ratio $Q_{0}$ in Catalytic Hydroamidation Reactions}

$$
\begin{aligned}
& \text { thiol }+ \text { amide }+ \text { base } \stackrel{K_{\text {eq }}}{\rightleftharpoons} \text { thiol adduct }+ \text { amide adduct } \\
& \begin{array}{lllll}
S & N & P & 0 & 0
\end{array} \\
& \begin{array}{lllll}
\text { S-x } & \mathrm{N}-\mathrm{y} & \mathrm{P}-\mathrm{x}-\mathrm{y} & \mathrm{x} & \mathrm{y}
\end{array} \\
& \text { thiol }+ \text { base } \stackrel{K_{\mathrm{A} \text { thiol }}}{=} \text { thiol adduct } \\
& \text { amide }+ \text { base } \stackrel{K_{\mathrm{A} \text { amide }}}{=} \text { amide adduct } \\
& \left\{\begin{aligned}
K_{\mathrm{A} \text { amide }} & =\frac{y}{(\mathrm{~N}-\mathrm{y})(\mathrm{P}-\mathrm{x}-\mathrm{y})} \\
K_{\mathrm{A} \text { thiol }} & =\frac{\mathrm{x}}{(\mathrm{S}-\mathrm{x})(\mathrm{P}-\mathrm{x}-\mathrm{y})}
\end{aligned}\right. \\
& \mathrm{Q}_{0}=\frac{k_{\mathrm{PCET} \text { amide }}[\text { amide adduct }]_{0}}{k_{\mathrm{PCET} \text { thiol }}[\text { thiol adduct }]_{0}}=\frac{k_{\mathrm{PCET} \text { amide }} \mathrm{y}}{k_{\mathrm{PCET} \text { thiol }} \mathrm{x}}
\end{aligned}
$$




\subsection{Table 2 in the main text}

Table S6.1. Correlate MS-PCET Kinetic, H-Bonding, and Selectivity Data with Catalytic Reaction Outcomes

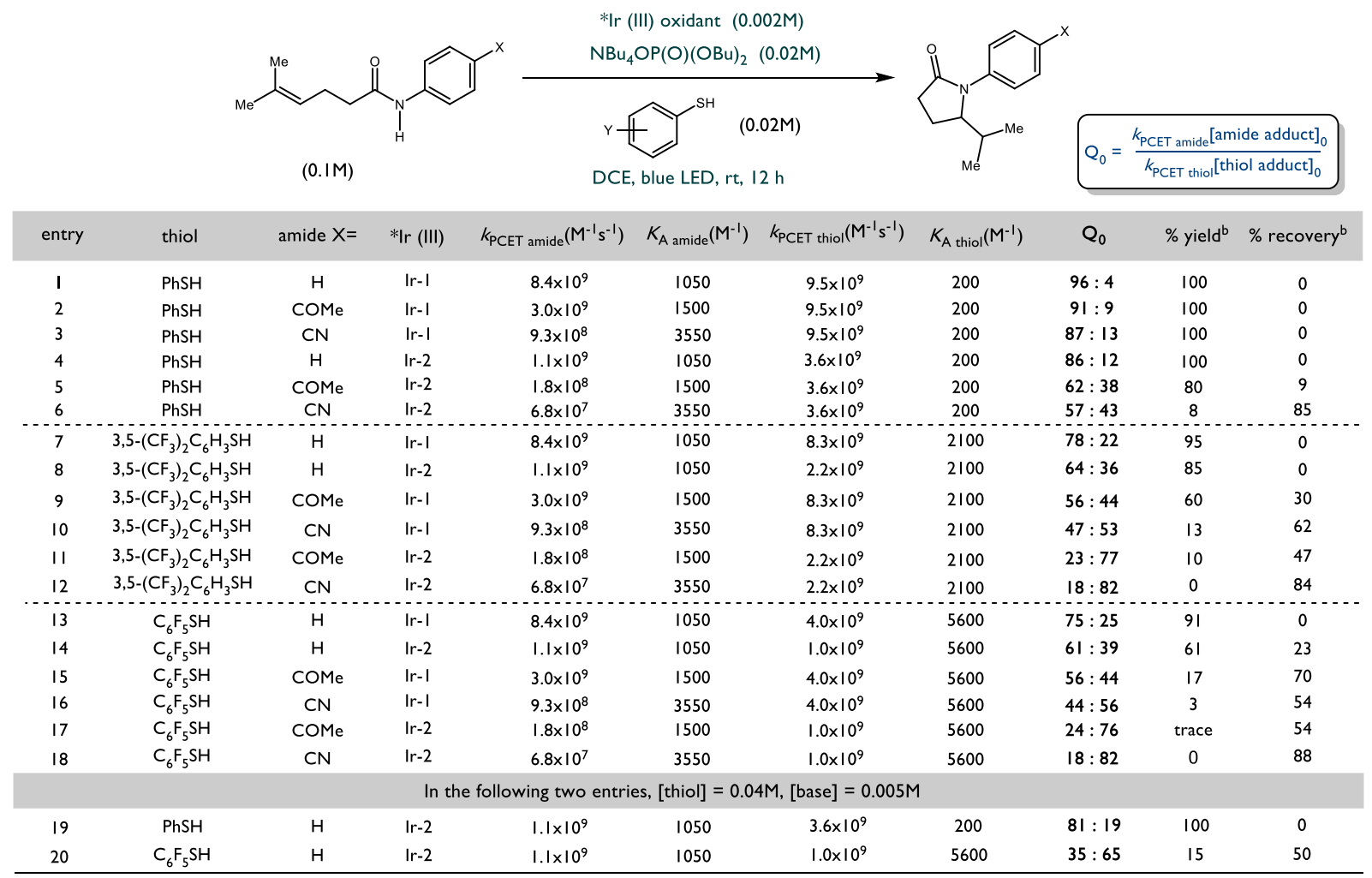

${ }^{a}$ All reactions are on the scale of $0.05 \mathrm{mmol}$. ${ }^{b}$ Each percentage yield and starting material recovery was taken after 12 hours of reaction time, by NMR relative to internal standard. There was no further product formation upon prolonged reaction time.

\subsection{1. entry 1}

$K_{\text {Aamide }}=1050 \mathrm{M}^{-1}, K_{\mathrm{A} \text { thiol }}=200 \mathrm{M}^{-1} \cdot k_{\mathrm{PCET} \text { amide }}=8.4 \times 10^{9} \mathrm{M}^{-1} \mathrm{~s}^{-1}, k_{\mathrm{PCET} \text { thiol }}=9.5 \times 10^{9} \mathrm{M}^{-1} \mathrm{~s}^{-1}$

$[\text { amide }]_{0}=0.10 \mathrm{M},[$ thiol $]=0.020 \mathrm{M},[$ Base $]=0.020 \mathrm{M}$

$$
\begin{gathered}
\frac{y}{(0.1-y)(0.02-x-y)}=1050 \\
\frac{x}{(0.02-x)(0.02-x-y)}=200 \\
\mathrm{y}=0.019 \mathrm{M}, \mathrm{x}=0.00085 \mathrm{M} \\
Q_{0}=\frac{k_{\text {PCET amide }[\text { amide adduct }]_{0}}}{k_{\text {PCET thiol }[\text { thiol adduct }]_{0}}}=\frac{8.4 \times 10^{9} \times 0.019}{9.5 \times 10^{9} \times 0.00085}=19.7=96: 4
\end{gathered}
$$




\subsection{2. entry 2}

$K_{\text {A amide }}=1500 \mathrm{M}^{-1}, K_{\text {A thiol }}=200 \mathrm{M}^{-1} \cdot k_{\mathrm{PCET} \text { amide }}=3.0 \times 10^{9} \mathrm{M}^{-1} \mathrm{~s}^{-1}, k_{\mathrm{PCET} \text { thiol }}=9.5 \times 10^{9} \mathrm{M}^{-1} \mathrm{~s}^{-1}$

$[\text { amide }]_{0}=0.10 \mathrm{M},[$ thiol $]=0.020 \mathrm{M},[$ Base $]=0.020 \mathrm{M}$

$$
\begin{aligned}
& \frac{y}{(0.1-y)(0.02-x-y)}=1450 \\
& \frac{x}{(0.02-x)(0.02-x-y)}=200 \\
& y=0.019 M, x=0.00062 M \\
& Q_{0}=\frac{k_{P C E T \text { amide }}[\text { amide adduct }]_{0}}{k_{\text {PCET thiol }}[\text { thiol adduct }]_{0}}=\frac{3.0 \times 10^{9} \times 0.0192}{9.5 \times 10^{9} \times 0.00065}=9.3=91: 9
\end{aligned}
$$

\subsection{4. entry 3}

$K_{\mathrm{A} \text { amide }}=3550 \mathrm{M}^{-1}, K_{\mathrm{A} \text { thiol }}=200 \mathrm{M}^{-1} \cdot k_{\mathrm{PCET} \text { amide }}=9.3 \times 10^{8} \mathrm{M}^{-1} \mathrm{~s}^{-1}, k_{\mathrm{PCET}}$ thiol $=9.5 \times 10^{9} \mathrm{M}^{-1} \mathrm{~s}^{-1}$

$[\text { amide }]_{0}=0.10 \mathrm{M},[$ thiol $]=0.020 \mathrm{M},[$ Base $]=0.020 \mathrm{M}$

$$
\begin{aligned}
& \frac{y}{(0.1-y)(0.02-x-y)}=3550 \\
& \frac{x}{(0.02-x)(0.02-x-y)}=200 \\
& y=0.020 M, x=0.00028 M
\end{aligned}
$$

$$
Q_{0}=\frac{k_{\text {PCET amide }}[\text { amide adduct }]_{0}}{k_{\text {PCET thiol }}[\text { thiol adduct }]_{0}}=\frac{9.3 \times 10^{8} \times 0.020}{9.5 \times 10^{9} \times 0.00028}=7.0=87: 13
$$




\subsection{3. entry 4}

$K_{\text {A amide }}=1050 \mathrm{M}^{-1}, K_{\text {A thiol }}=200 \mathrm{M}^{-1} \cdot k_{\text {PCET amide }}=1.1 \times 10^{9} \mathrm{M}^{-1} \mathrm{~s}^{-1}, k_{\text {PCET thiol }}=3.6 \times 10^{9} \mathrm{M}^{-1} \mathrm{~s}^{-1}$

$[\text { amide }]_{0}=0.10 \mathrm{M},[$ thiol $]=0.020 \mathrm{M},[$ Base $]=0.020 \mathrm{M}$

$$
\begin{aligned}
& \frac{y}{(0.1-y)(0.02-x-y)}=1050 \\
& \frac{x}{(0.02-x)(0.02-x-y)}=200 \\
& y=0.019 M, x=0.00085 M \\
& Q_{0}=\frac{k_{\text {PCET amide }}[\text { amide adduct }]_{0}}{k_{\text {PCET thiol }}[\text { thiol adduct }]_{0}}=\frac{1.1 \times 10^{9} \times 0.019}{3.6 \times 10^{9} \times 0.00085}=6.8=86: 12
\end{aligned}
$$

\subsection{5. entry 5}

$K_{\text {A amide }}=1500 \mathrm{M}^{-1}, K_{\text {A thiol }}=200 \mathrm{M}^{-1} \cdot k_{\text {PCET amide }}=1.8 \times 10^{8} \mathrm{M}^{-1} \mathrm{~s}^{-1}, k_{\text {PCET thiol }}=3.6 \times 10^{9} \mathrm{M}^{-1} \mathrm{~s}^{-1}$

$[\text { amide }]_{0}=0.10 \mathrm{M},[$ thiol $]=0.020 \mathrm{M},[$ Base $]=0.020 \mathrm{M}$

$$
\begin{aligned}
& \frac{y}{(0.1-y)(0.02-x-y)}=1450 \\
& \frac{x}{(0.02-x)(0.02-x-y)}=200 \\
& y=0.0192 M, x=0.000650 M \\
& Q_{0}=\frac{k_{\text {PCET amide }[\text { amide adduct }]_{0}}}{k_{\text {PCET thiol }}[\text { thiol adduct }]_{0}}=\frac{1.8 \times 10^{8} \times 0.0192}{3.6 \times 10^{9} \times 0.00065}=1.5=61: 39
\end{aligned}
$$

\subsection{6. entry 6}

$K_{\text {A amide }}=3550 \mathrm{M}^{-1}, K_{\text {A thiol }}=200 \mathrm{M}^{-1} \cdot k_{\text {PCET amide }}=6.8 \times 10^{7} \mathrm{M}^{-1} \mathrm{~s}^{-1}, k_{\text {PCET thiol }}=3.6 \times 10^{9} \mathrm{M}^{-1} \mathrm{~s}^{-1}$

$[\text { amide }]_{0}=0.10 \mathrm{M},[$ thiol $]=0.020 \mathrm{M},[$ Base $]=0.020 \mathrm{M}$

$$
\begin{aligned}
& \frac{y}{(0.1-y)(0.02-x-y)}=3550 \\
& \frac{x}{(0.02-x)(0.02-x-y)}=200
\end{aligned}
$$




$$
\begin{gathered}
\mathrm{y}=0.020 \mathrm{M}, \mathrm{x}=0.00028 \mathrm{M} \\
Q_{0}=\frac{k_{\text {PCET amide }}[\text { amide adduct }]_{0}}{k_{\text {PCET thiol }[\text { thiol adduct }]_{0}}}=\frac{6.8 \times 10^{7} \times 0.020}{3.6 \times 10^{9} \times 0.00028}=1.3=57: 43
\end{gathered}
$$

\subsection{7. entry 7}

$$
K_{\text {A amide }}=1050 \mathrm{M}^{-1}, K_{\text {A thiol }}=2150 \mathrm{M}^{-1} \cdot k_{\mathrm{PCET} \text { amide }}=8.4 \times 10^{9} \mathrm{M}^{-1} \mathrm{~s}^{-1}, k_{\mathrm{PCET} \text { thiol }}=8.3 \times 10^{9} \mathrm{M}^{-1} \mathrm{~s}^{-1}
$$

$[\text { amide }]_{0}=0.10 \mathrm{M},[$ thiol $]=0.016 \mathrm{M},[$ Base $]=0.017 \mathrm{M}$

$$
\begin{gathered}
\frac{y}{(0.1-y)(0.017-x-y)}=1050 \\
\frac{x}{(0.016-x)(0.017-x-y)}=2100 \\
\mathrm{y}=0.013 \mathrm{M}, \mathrm{x}=0.0037 \mathrm{M} \\
Q_{0}=\frac{k_{\text {PCET amide }[\text { amide adduct }]_{0}}}{k_{\text {PCET thiol }[\text { thiol adduct }]_{0}}}=\frac{8.4 \times 10^{9} \times 0.013}{8.3 \times 10^{9} \times 0.0037}=3.6=78: 22
\end{gathered}
$$

6.1.8. entry 8

$K_{\mathrm{A} \text { amide }}=1050 \mathrm{M}^{-1}, K_{\mathrm{A} \text { thiol }}=2100 \mathrm{M}^{-1} \cdot k_{\mathrm{PCET} \text { amide }}=1.1 \times 10^{9} \mathrm{M}^{-1} \mathrm{~s}^{-1}, k_{\mathrm{PCET} \text { thiol }}=2.2 \times 10^{9} \mathrm{M}^{-1} \mathrm{~s}^{-1}$

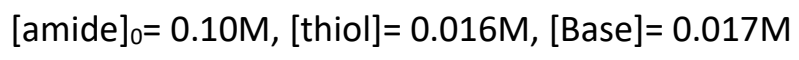

$$
\begin{aligned}
& \frac{y}{(0.1-y)(0.017-x-y)}=1050 \\
& \frac{x}{(0.016-x)(0.017-x-y)}=2100 \\
& y=0.013 M, x=0.0037 M \\
& Q_{0}=\frac{k_{P C E T \text { amide }}[\text { amide adduct }]_{0}}{k_{\text {PCET thiol }[\text { thiol adduct }]_{0}}}=\frac{1.1 \times 10^{9} \times 0.013}{2.2 \times 10^{9} \times 0.0037}=1.8=64: 36
\end{aligned}
$$

\subsection{9. entry 9}

$K_{\text {A amide }}=1500 \mathrm{M}^{-1}, K_{\text {A thiol }}=2150 \mathrm{M}^{-1} \cdot k_{\text {PCET amide }}=3.0 \times 10^{9} \mathrm{M}^{-1} \mathrm{~s}^{-1}, k_{\text {PCET thiol }}=8.3 \times 10^{9} \mathrm{M}^{-1} \mathrm{~s}^{-1}$

$[\text { amide }]_{0}=0.10 \mathrm{M},[$ thiol $]=0.020 \mathrm{M},[$ Base $]=0.020 \mathrm{M}$ 


$$
\begin{gathered}
\frac{y}{(0.1-y)(0.02-x-y)}=1450 \\
\frac{x}{(0.02-x)(0.02-x-y)}=2100 \\
\mathrm{y}=0.0156 \mathrm{M}, \mathrm{x}=0.00423 \mathrm{M} \\
Q_{0}=\frac{k_{\text {PCET amide }[\text { amide adduct }]_{0}}}{k_{\text {PCET thiol }[\text { thiol adduct }]_{0}}}=\frac{3.0 \times 10^{9} \times 0.0156}{8.3 \times 10^{9} \times 0.00423}=1.3=56: 44
\end{gathered}
$$

\subsubsection{0. entry 10}

$K_{\text {A amide }}=3550 \mathrm{M}^{-1}, K_{\text {A thiol }}=2150 \mathrm{M}^{-1} \cdot k_{\text {PCET amide }}=9.3 \times 10^{8} \mathrm{M}^{-1} \mathrm{~s}^{-1}, k_{\mathrm{PCET} \text { thiol }}=8.3 \times 10^{9} \mathrm{M}^{-1} \mathrm{~s}^{-1}$

$[\text { amide }]_{0}=0.10 \mathrm{M},[$ thiol $]=0.020 \mathrm{M},[$ Base $]=0.020 \mathrm{M}$

$$
\begin{aligned}
& \frac{y}{(0.1-y)(0.02-x-y)}=3550 \\
& \frac{x}{(0.02-x)(0.02-x-y)}=2100 \\
& y=0.0177 M, x=0.00226 M \\
& Q_{0}=\frac{k_{\text {PCET amide }}[\text { amide adduct }]_{0}}{k_{\text {PCET thiol }}[\text { thiol adduct }]_{0}}=\frac{9.3 \times 10^{8} \times 0.0177}{8.3 \times 10^{9} \times 0.00226}=0.88=47: 53
\end{aligned}
$$

\subsubsection{1. entry 11}

$K_{\text {A amide }}=1500 \mathrm{M}^{-1}, K_{\text {A thiol }}=2150 \mathrm{M}^{-1} \cdot k_{\text {PCET amide }}=1.8 \times 10^{8} \mathrm{M}^{-1} \mathrm{~s}^{-1}, k_{\text {PCET thiol }}=2.2 \times 10^{9} \mathrm{M}^{-1} \mathrm{~s}^{-1}$

$[\text { amide }]_{0}=0.10 \mathrm{M},[$ thiol $]=0.020 \mathrm{M},[$ Base $]=0.020 \mathrm{M}$

$$
\begin{aligned}
& \frac{y}{(0.1-y)(0.02-x-y)}=1450 \\
& \frac{x}{(0.02-x)(0.02-x-y)}=2100 \\
& y=0.0156 \mathrm{M}, \mathrm{x}=0.00423 \mathrm{M} \\
& Q_{0}=\frac{k_{\text {PCET amide }}[\text { amide adduct }]_{0}}{k_{\text {PCET thiol }}[\text { thiol adduct }]_{0}}=\frac{1.8 \times 10^{8} \times 0.0156}{2.2 \times 10^{9} \times 0.00423}=0.30=23: 77
\end{aligned}
$$




\subsubsection{2. entry 12}

$K_{\text {Aamide }}=3550 \mathrm{M}^{-1}, K_{\mathrm{A} \text { thiol }}=2150 \mathrm{M}^{-1} \cdot k_{\text {PCET amide }}=6.8 \times 10^{7} \mathrm{M}^{-1} \mathrm{~s}^{-1}, k_{\text {PCET thiol }}=2.2 \times 10^{9} \mathrm{M}^{-1} \mathrm{~s}^{-1}$

$[\text { amide }]_{0}=0.10 \mathrm{M},[$ thiol $]=0.020 \mathrm{M},[$ Base $]=0.020 \mathrm{M}$

$$
\begin{aligned}
& \frac{y}{(0.1-y)(0.02-x-y)}=3550 \\
& \frac{x}{(0.02-x)(0.02-x-y)}=2100 \\
& y=0.0177 M, x=0.00226 M \\
& Q_{0}=\frac{k_{\text {PCET amide }}[\text { amide adduct }]_{0}}{k_{\text {PCET thiol }}[\text { thiol adduct }]_{0}}=\frac{6.8 \times 10^{7} \times 0.0177}{2.2 \times 10^{9} \times 0.00226}=0.22=18: 82
\end{aligned}
$$

\subsubsection{3. entry 13}

$$
K_{\text {A amide }}=1050 \mathrm{M}^{-1}, K_{\text {A thiol }}=5600 \mathrm{M}^{-1} \cdot k_{\text {PCET amide }}=8.4 \times 10^{9} \mathrm{M}^{-1} \mathrm{~s}^{-1}, k_{\mathrm{PCET} \text { thiol }}=4.0 \times 10^{9} \mathrm{M}^{-1} \mathrm{~s}^{-1}
$$

$[\text { amide }]_{0}=0.10 \mathrm{M},[$ thiol $]=0.020 \mathrm{M},[$ Base $]=0.020 \mathrm{M}$

$$
\begin{gathered}
\frac{y}{(0.1-y)(0.02-x-y)}=1050 \\
\frac{x}{(0.02-x)(0.02-x-y)}=5600 \\
\mathrm{y}=0.0116 \mathrm{M}, \mathrm{x}=0.00825 \mathrm{M} \\
Q_{0}=\frac{k_{\text {PCET amide }[\text { amide adduct }]_{0}}}{k_{\text {PCET thiol }[\text { thiol adduct }]_{0}}}=\frac{8.4 \times 10^{9} \times 0.0116}{4.0 \times 10^{9} \times 0.00825}=3.0=75: 25
\end{gathered}
$$

\subsubsection{4. entry 14}

$K_{\text {A amide }}=1050 \mathrm{M}^{-1}, K_{\text {A thiol }}=5600 \mathrm{M}^{-1} \cdot k_{\text {PCET amide }}=1.1 \times 10^{9} \mathrm{M}^{-1} \mathrm{~s}^{-1}, k_{\text {PCET thiol }}=1.0 \times 10^{9} \mathrm{M}^{-1} \mathrm{~s}^{-1}$

$[\text { amide }]_{0}=0.10 \mathrm{M},[$ thiol $]=0.020 \mathrm{M},[$ Base $]=0.020 \mathrm{M}$

$$
\begin{aligned}
& \frac{y}{(0.1-y)(0.02-x-y)}=1050 \\
& \frac{x}{(0.02-x)(0.02-x-y)}=5600 \\
& y=0.0116 \mathrm{M}, \mathrm{x}=0.00825 \mathrm{M}
\end{aligned}
$$




$$
Q_{0}=\frac{k_{\text {PCET amide }}[\text { amide adduct }]_{0}}{k_{\text {PCET thiol }}[\text { thiol adduct }]_{0}}=\frac{1.1 \times 10^{9} \times 0.0116}{1.0 \times 10^{9} \times 0.00825}=1.5=61: 39
$$

\subsubsection{5. entry 15}

$K_{\text {A amide }}=1500 \mathrm{M}^{-1}, K_{\text {A thiol }}=5600 \mathrm{M}^{-1} \cdot k_{\text {PCET amide }}=3.0 \times 10^{9} \mathrm{M}^{-1} \mathrm{~s}^{-1}, k_{\text {PCET thiol }}=4.0 \times 10^{9} \mathrm{M}^{-1} \mathrm{~s}^{-1}$

$[\text { amide }]_{0}=0.10 \mathrm{M},[$ thiol $]=0.020 \mathrm{M},[$ Base $]=0.020 \mathrm{M}$

$$
\begin{gathered}
\frac{y}{(0.1-y)(0.02-x-y)}=1450 \\
\frac{x}{(0.02-x)(0.02-x-y)}=5600 \\
\mathrm{y}=0.0127 \mathrm{M}, \mathrm{x}=0.00720 \mathrm{M} \\
Q_{0}=\frac{k_{\text {PCET amide }[\text { amide adduct }]_{0}}}{k_{\text {PCET thiol }[\text { thiol adduct }]_{0}}}=\frac{3.0 \times 10^{9} \times 0.0127}{4.0 \times 10^{9} \times 0.00720}=1.3=56: 44
\end{gathered}
$$

\subsubsection{6. entry 16}

$K_{\text {A amide }}=3550 \mathrm{M}^{-1}, K_{\mathrm{A} \text { thiol }}=5600 \mathrm{M}^{-1} \cdot k_{\mathrm{PCET} \text { amide }}=9.3 \times 10^{8} \mathrm{M}^{-1} \mathrm{~s}^{-1}, k_{\mathrm{PCET} \text { thiol }}=4.0 \times 10^{9} \mathrm{M}^{-1} \mathrm{~s}^{-1}$

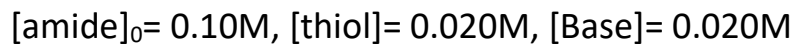

$$
\begin{aligned}
& \frac{y}{(0.1-y)(0.02-x-y)}=3550 \\
& \frac{x}{(0.02-x)(0.02-x-y)}=5600 \\
& y=0.0154 M, x=0.00448 M \\
& Q_{0}=\frac{k_{P C E T \text { amide }}[\text { amide adduct }]_{0}}{k_{\text {PCET thiol }}[\text { thiol adduct }]_{0}}=\frac{9.3 \times 10^{8} \times 0.0154}{4.0 \times 10^{9} \times 0.00448}=0.79=44: 56
\end{aligned}
$$

\subsubsection{7. entry 17}

$K_{\text {A amide }}=1500 \mathrm{M}^{-1}, K_{\text {A thiol }}=5600 \mathrm{M}^{-1} \cdot k_{\text {PCET amide }}=1.8 \times 10^{8} \mathrm{M}^{-1} \mathrm{~s}^{-1}, k_{\mathrm{PCET} \text { thiol }}=1.0 \times 10^{9} \mathrm{M}^{-1} \mathrm{~s}^{-1}$ 
$[\text { amide }]_{0}=0.10 \mathrm{M},[$ thiol $]=0.020 \mathrm{M},[$ Base $]=0.020 \mathrm{M}$

$$
\begin{aligned}
& \frac{y}{(0.1-y)(0.02-x-y)}=1450 \\
& \frac{x}{(0.02-x)(0.02-x-y)}=5600 \\
& y=0.0127 M, x=0.00720 M \\
& Q_{0}=\frac{k_{P C E T \text { amide }}[\text { amide adduct }]_{0}}{k_{\text {PCET thiol }}[\text { thiol adduct }]_{0}}=\frac{1.8 \times 10^{8} \times 0.0127}{1.0 \times 10^{9} \times 0.00720}=0.32=24: 76
\end{aligned}
$$

\subsubsection{8. entry 18}

$K_{\mathrm{A} \text { amide }}=3550 \mathrm{M}^{-1}, K_{\mathrm{A} \text { thiol }}=5600 \mathrm{M}^{-1} \cdot k_{\mathrm{PCET} \text { amide }}=6.8 \times 10^{7} \mathrm{M}^{-1} \mathrm{~s}^{-1}, k_{\mathrm{PCET}}$ thiol $=1.0 \times 10^{9} \mathrm{M}^{-1} \mathrm{~s}^{-1}$

$[\text { amide }]_{0}=0.10 \mathrm{M},[$ thiol $]=0.020 \mathrm{M},[$ Base $]=0.020 \mathrm{M}$

$$
\begin{aligned}
& \frac{y}{(0.1-y)(0.02-x-y)}=3550 \\
& \frac{x}{(0.02-x)(0.02-x-y)}=5600 \\
& y=0.0154 M, x=0.00448 M \\
& Q_{0}=\frac{k_{\text {PCET amide }}[\text { amide adduct }]_{0}}{k_{\text {PCET thiol }}[\text { thiol adduct }]_{0}}=\frac{6.8 \times 10^{7} \times 0.0154}{1.0 \times 10^{9} \times 0.00448}=0.21=18: 82
\end{aligned}
$$

\subsection{1. entry 19}

$K_{\text {A amide }}=1050 \mathrm{M}^{-1}, K_{\mathrm{A} \text { thiol }}=200 \mathrm{M}^{-1} \cdot k_{\mathrm{PCET} \text { amide }}=1.1 \times 10^{9} \mathrm{M}^{-1} \mathrm{~s}^{-1}, k_{\mathrm{PCET}}$ thiol $=3.6 \times 10^{9} \mathrm{M}^{-1} \mathrm{~s}^{-1}$

$[\text { amide }]_{0}=0.10 \mathrm{M},[$ thiol $]=0.040 \mathrm{M},[$ Base $]=0.0050 \mathrm{M}$

$$
\begin{gathered}
\frac{y}{(0.1-y)(0.005-x-y)}=1050 \\
\frac{x}{(0.04-x)(0.005-x-y)}=200 \\
\mathrm{y}=0.0050 \mathrm{M}, \mathrm{x}=0.00036 \mathrm{M} \\
Q_{0}=\frac{k_{\text {PCET amide }[\text { amide adduct }]_{0}}}{k_{\text {PCET thiol }[\text { thiol adduct }]_{0}}}=\frac{1.1 \times 10^{9} \times 0.005}{3.6 \times 10^{9} \times 0.00036}=4.2=81: 19
\end{gathered}
$$


6.1.1. entry 20

$K_{\text {A amide }}=1050 \mathrm{M}^{-1}, K_{\text {A thiol }}=5600 \mathrm{M}^{-1} \cdot k_{\text {PCET amide }}=1.1 \times 10^{9} \mathrm{M}^{-1} \mathrm{~s}^{-1}, k_{\text {PCET thiol }}=1.0 \times 10^{9} \mathrm{M}^{-1} \mathrm{~s}^{-1}$

$[\text { amide }]_{0}=0.10 \mathrm{M},[$ thiol $]=0.040 \mathrm{M},[$ Base $]=0.0050 \mathrm{M}$

$$
\begin{gathered}
\frac{y}{(0.1-y)(0.005-x-y)}=1050 \\
\frac{x}{(0.04-x)(0.005-x-y)}=5600 \\
\mathrm{y}=0.00167 \mathrm{M}, \mathrm{x}=0.0033 \mathrm{M} \\
Q_{0}=\frac{k_{\text {PCET amide }[\text { amide adduct }]_{0}}}{k_{\text {PCET thiol }[\text { thiol adduct }]_{0}}}=\frac{1.1 \times 10^{9} \times 0.00167}{1.0 \times 10^{9} \times 0.0033}=0.55=35: 65
\end{gathered}
$$




\subsection{Manipulation of Reaction to Attain a Broad Range of Yields by Varying Base Loading}

To minimize effects other than MS-PCET selectivity on the hydroamidation yield, we attained a range of $Q_{0}$ values by only varying the loading of base, under identical conditions otherwise, as attempts to obtain a range of moderate reaction yields. The reaction outcomes are presented in Table S6.2. A broad range of yield from $22 \%$ to $88 \%$ were attained and the yield well correlated with $Q_{0}$. Besides lower product yields, higher loading of thiol also resulted in higher substrate recoveries and better mass balances. These results are consistent with our hypothesis: MS-PCET selectivity is vital for the product formation. Beyond the endpoint of hydroamidation product formation, the MS-PCET chemoselectivity towards thiol would protect the amide from decomposition.

Table S6.2. Manipulation of Reaction to Attain a Broad Range of Yields

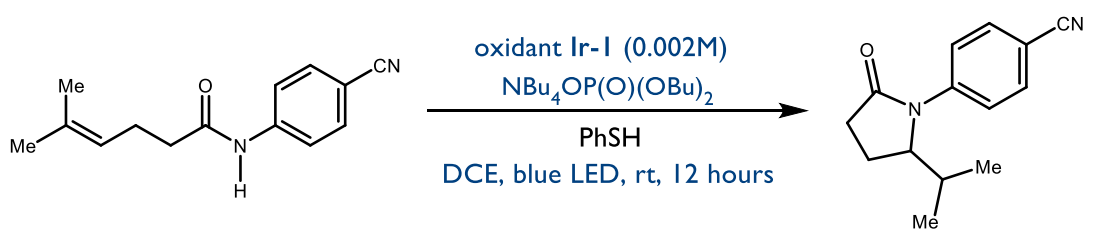

\begin{tabular}{ccccccc} 
entry & [amide]/M & [thiol] $/ M$ & {$[$ base]/M } & ratio $Q_{0}$ & $\%$ yield \% recovery \\
\hline & 0.05 & 0.02 & 0.005 & $80: 20$ & 88 & 4 \\
2 & 0.05 & 0.02 & 0.02 & $73: 27$ & 81 & 0 \\
3 & 0.05 & 0.02 & 0.04 & $57: 43$ & 74 & 4 \\
4 & 0.05 & 0.02 & 0.06 & $34: 66$ & 48 & 14 \\
\hline 5 & 0.05 & 0.09 & 0.01 & $44: 56$ & 62 & 25 \\
6 & 0.05 & 0.09 & 0.03 & $34: 66$ & 50 & 45 \\
7 & 0.05 & 0.09 & 0.05 & $23: 77$ & 40 & 60 \\
8 & 0.05 & 0.09 & 0.07 & $14: 86$ & 22 & 66 \\
\hline
\end{tabular}

All reactions are on the scale of $0.05 \mathrm{mmol}$. Each percentage yield was taken after 12 hours of reaction time, by NMR. There was no further product formation upon prolonged reaction time.

\subsection{1. entry 1}

$K_{\text {A amide }}=3550 \mathrm{M}^{-1}, K_{\text {A thiol }}=200 \mathrm{M}^{-1} \cdot k_{\text {PCET amide }}=9.3 \times 10^{8} \mathrm{M}^{-1} \mathrm{~s}^{-1}, k_{\text {PCET thiol }}=9.5 \times 10^{9} \mathrm{M}^{-1} \mathrm{~s}^{-1}$

$[\text { amide }]_{0}=0.050 \mathrm{M},[$ thiol $]=0.020 \mathrm{M},[$ Base $]=0.0050 \mathrm{M}$

$$
\begin{gathered}
\frac{y}{(0.05-y)(0.005-x-y)}=3550 \\
\frac{x}{(0.02-x)(0.005-x-y)}=200 \\
y=0.00485 \mathrm{M}, \mathrm{x}=0.00012 \mathrm{M}
\end{gathered}
$$




$$
Q_{0}=\frac{k_{\text {PCET amide }}[\text { amide adduct }]_{0}}{k_{\text {PCET thiol }}[\text { thiol adduct }]_{0}}=\frac{9.3 \times 10^{8} \times 0.00485}{9.5 \times 10^{9} \times 0.00012}=4.0=80: 20
$$

\subsection{2. entry 2}

$K_{\text {A amide }}=3550 \mathrm{M}^{-1}, K_{\text {A thiol }}=200 \mathrm{M}^{-1} \cdot k_{\text {PCET amide }}=9.3 \times 10^{8} \mathrm{M}^{-1} \mathrm{~s}^{-1}, k_{\text {PCET thiol }}=9.5 \times 10^{9} \mathrm{M}^{-1} \mathrm{~s}^{-1}$

[amide $_{0}=0.050 \mathrm{M},[$ thiol $]=0.020 \mathrm{M},[$ Base $]=0.020 \mathrm{M}$

$$
\begin{gathered}
\frac{y}{(0.05-y)(0.02-x-y)}=3550 \\
\frac{x}{(0.02-x)(0.02-x-y)}=200 \\
\mathrm{y}=0.0192 \mathrm{M}, \mathrm{x}=0.00068 \mathrm{M} \\
Q_{0}=\frac{k_{\text {PCET amide }[\text { amide adduct }]_{0}}}{k_{\text {PCET thiol }[\text { thiol adduct }]_{0}}}=\frac{9.3 \times 10^{8} \times 0.0192}{9.5 \times 10^{9} \times 0.00068}=2.8=73: 27
\end{gathered}
$$

\subsection{3. entry 3}

$K_{\text {A amide }}=3550 \mathrm{M}^{-1}, K_{\text {A thiol }}=200 \mathrm{M}^{-1} \cdot k_{\text {PCET amide }}=9.3 \times 10^{8} \mathrm{M}^{-1} \mathrm{~s}^{-1}, k_{\text {PCET thiol }}=9.5 \times 10^{9} \mathrm{M}^{-1} \mathrm{~s}^{-1}$

$[\text { amide }]_{0}=0.050 \mathrm{M},[$ thiol $]=0.020 \mathrm{M},[$ Base $]=0.040 \mathrm{M}$

$$
\begin{aligned}
& \frac{y}{(0.05-y)(0.04-x-y)}=3550 \\
& \frac{x}{(0.02-x)(0.04-x-y)}=200 \\
& y=0.0366 \mathrm{M}, x=0.000266 \mathrm{M} \\
& Q_{0}=\frac{k_{\text {PCET amide }}[\text { amide adduct }]_{0}}{k_{\text {PCET thiol }}[\text { thiol adduct }]_{0}}=\frac{9.3 \times 10^{8} \times 0.0366}{9.5 \times 10^{9} \times 0.00266}=1.3=57: 43
\end{aligned}
$$

\subsection{4. entry 4}

$K_{\text {A amide }}=3550 \mathrm{M}^{-1}, K_{\text {A thiol }}=200 \mathrm{M}^{-1} \cdot k_{\text {PCET amide }}=9.3 \times 10^{8} \mathrm{M}^{-1} \mathrm{~s}^{-1}, k_{\text {PCET thiol }}=9.5 \times 10^{9} \mathrm{M}^{-1} \mathrm{~s}^{-1}$

$[\text { amide }]_{0}=0.050 \mathrm{M},[$ thiol $]=0.020 \mathrm{M},[$ Base $]=0.060 \mathrm{M}$ 


$$
\begin{gathered}
\frac{y}{(0.05-y)(0.06-x-y)}=3550 \\
\frac{x}{(0.02-x)(0.06-x-y)}=200 \\
\mathrm{y}=0.0468 \mathrm{M}, \mathrm{x}=0.00905 \mathrm{M} \\
Q_{0}=\frac{k_{\text {PCET amide }[\text { amide adduct }]_{0}}}{k_{\text {PCET thiol }}[\text { thiol adduct }]_{0}}=\frac{9.3 \times 10^{8} \times 0.0468}{9.5 \times 10^{9} \times 0.00905}=0.51=34: 66
\end{gathered}
$$

\subsection{5. entry 5}

$K_{\text {A amide }}=3550 \mathrm{M}^{-1}, K_{\text {A thiol }}=200 \mathrm{M}^{-1} \cdot k_{\text {PCET amide }}=9.3 \times 10^{8} \mathrm{M}^{-1} \mathrm{~s}^{-1}, k_{\text {PCET thiol }}=9.5 \times 10^{9} \mathrm{M}^{-1} \mathrm{~s}^{-1}$

[amide $_{0}=0.050 \mathrm{M},[$ thiol $]=0.090 \mathrm{M},[$ Base $]=0.010 \mathrm{M}$

$$
\begin{aligned}
& \frac{y}{(0.05-y)(0.01-x-y)}=3550 \\
& \frac{x}{(0.09-x)(0.01-x-y)}=200 \\
& y=0.0089 M, x=0.0011 M \\
& Q_{0}=\frac{k_{P C E T \text { amide }}[\text { amide adduct }]_{0}}{k_{\text {PCET thiol }}[\text { thiol adduct }]_{0}}=\frac{9.3 \times 10^{8} \times 0.0089}{9.5 \times 10^{9} \times 0.0011}=0.79=44: 56
\end{aligned}
$$

\subsection{6. entry 6}

$K_{\text {A amide }}=3550 \mathrm{M}^{-1}, K_{\text {A thiol }}=200 \mathrm{M}^{-1} \cdot k_{\text {PCET amide }}=9.3 \times 10^{8} \mathrm{M}^{-1} \mathrm{~s}^{-1}, k_{\text {PCET thiol }}=9.5 \times 10^{9} \mathrm{M}^{-1} \mathrm{~s}^{-1}$

$[\text { amide }]_{0}=0.050 \mathrm{M},[$ thiol $]=0.090 \mathrm{M},[$ Base $]=0.030 \mathrm{M}$

$$
\begin{gathered}
\frac{y}{(0.05-y)(0.03-x-y)}=3550 \\
\frac{x}{(0.09-x)(0.03-x-y)}=200 \\
\mathrm{y}=0.025 \mathrm{M}, \mathrm{x}=0.0048 \mathrm{M} \\
Q_{0}=\frac{k_{\text {PCET amide }[\text { amide adduct }]_{0}}}{k_{\text {PCET thiol }[\text { thiol adduct }]_{0}}}=\frac{9.3 \times 10^{8} \times 0.025}{9.5 \times 10^{9} \times 0.0048}=0.51=34: 66
\end{gathered}
$$




\subsection{7. entry 7}

$K_{\mathrm{A} \text { amide }}=3550 \mathrm{M}^{-1}, K_{\mathrm{A} \text { thiol }}=200 \mathrm{M}^{-1} \cdot k_{\text {PCET amide }}=9.3 \times 10^{8} \mathrm{M}^{-1} \mathrm{~s}^{-1}, k_{\text {PCET thiol }}=9.5 \times 10^{9} \mathrm{M}^{-1} \mathrm{~s}^{-1}$

$[\text { amide }]_{0}=0.050 \mathrm{M},[$ thiol $]=0.090 \mathrm{M},[$ Base $]=0.050 \mathrm{M}$

$$
\begin{aligned}
& \frac{y}{(0.05-y)(0.05-x-y)}=3550 \\
& \frac{x}{(0.09-x)(0.05-x-y)}=200 \\
& y=0.037 M, x=0.012 M \\
& Q_{0}=\frac{k_{\text {PCET amide }}[\text { amide adduct }]_{0}}{k_{\text {PCET thiol }}[\text { thiol adduct }]_{0}}=\frac{9.3 \times 10^{8} \times 0.037}{9.5 \times 10^{9} \times 0.012}=0.30=23: 77
\end{aligned}
$$

\subsection{8. entry 8}

$K_{\text {A amide }}=3550 \mathrm{M}^{-1}, K_{\text {A thiol }}=200 \mathrm{M}^{-1} \cdot k_{\text {PCET amide }}=9.3 \times 10^{8} \mathrm{M}^{-1} \mathrm{~s}^{-1}, k_{\text {PCET thiol }}=9.5 \times 10^{9} \mathrm{M}^{-1} \mathrm{~s}^{-1}$

[amide $_{0}=0.050 \mathrm{M},[$ thiol $]=0.090 \mathrm{M},[$ Base $]=0.070 \mathrm{M}$

$$
\begin{aligned}
& \frac{y}{(0.05-y)(0.07-x-y)}=3550 \\
& \frac{x}{(0.09-x)(0.07-x-y)}=200 \\
& y=0.043 M, x=0.025 M \\
& Q_{0}=\frac{k_{\text {PCET amide }}[\text { amide adduct }]_{0}}{k_{\text {PCET thiol }}[\text { thiol adduct }]_{0}}=\frac{9.3 \times 10^{8} \times 0.043}{9.5 \times 10^{9} \times 0.025}=0.17=14: 86
\end{aligned}
$$




\section{Deconvolution of the Effect from Thiol-Related Steps Downstream in the Catalytic Cycle}

Table 2 in the main text shows that the threshold value of $Q$ below which hydroamidation becomes inhibited varies as a function of the thiol employed (e.g. entry 5 versus 9 and 15). Thus, the change in reaction yield with thiol identity is likely the result of both the changes in MS-PCET chemoselectivity and other thiol-related steps downstream in the catalytic cycle. Therefore, we attempted to deconvolute these effects and isolate the role of MS-PCET chemoselectivity on hydroamidation efficiency. In Table S7, the only difference among entries 1-3 (and 6-8) is the identity of the thiol. Upon adding the very effective HAT catalyst thiol T1, the yields are essentially entries 4 and 5, and are only partially rescued in entries 9 and 10 . These results are consistent with the notion that MS-PCET selectivity $\left(Q_{0}\right)$ is responsible for the remaining yield suppression.

Table S7. Catalytic Reactions with Dual Thiols

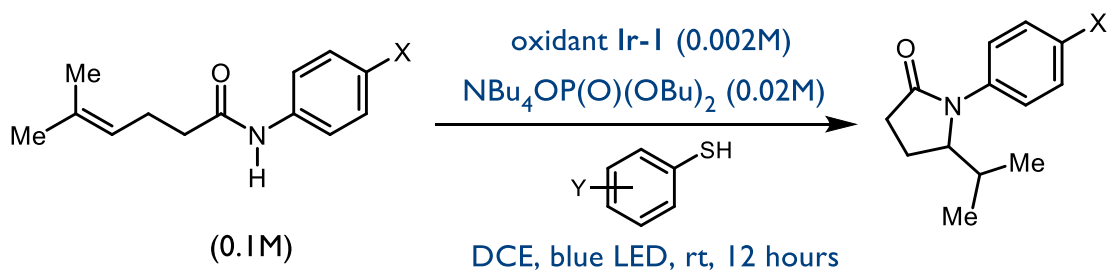

\begin{tabular}{ccccccc} 
entry & amide $\mathrm{X}=$ & thiol(s) & [thiol(s)]/M & ratio $\mathrm{Q}_{0}$ & $\%$ yield & $\%$ rec \\
\hline $\mathrm{I}$ & $\mathrm{CN}$ & $\mathrm{TI}$ & 0.02 & $88: 12$ & 100 & 0 \\
2 & $\mathrm{CN}$ & $\mathrm{T} 3$ & 0.02 & $44: 56$ & 3 & 54 \\
3 & $\mathrm{CN}$ & $\mathrm{T} 4$ & 0.02 & $47: 53$ & 13 & 62 \\
4 & $\mathrm{CN}$ & $\mathrm{TI}+\mathrm{T} 3$ & $0.02+0.02$ & $<44: 56$ & 4 & 68 \\
5 & $\mathrm{CN}$ & $\mathrm{TI}+\mathrm{T} 4$ & $0.02+0.02$ & $<47: 53$ & 20 & 64 \\
\hline 6 & $\mathrm{COMe}$ & $\mathrm{TI}$ & 0.02 & $91: 9$ & 100 & 0 \\
7 & $\mathrm{COMe}$ & $\mathrm{T} 3$ & 0.02 & $56: 44$ & 17 & 70 \\
8 & $\mathrm{COMe}$ & $\mathrm{T} 4$ & 0.02 & $56: 44$ & 60 & 30 \\
9 & $\mathrm{COMe}$ & $\mathrm{TI}+\mathrm{T} 3$ & $0.02+0.02$ & $<56: 44$ & 30 & 42 \\
10 & $\mathrm{COMe}$ & $\mathrm{TI}+\mathrm{T} 4$ & $0.02+0.02$ & $<56: 44$ & 77 & 0 \\
\hline
\end{tabular}




\section{References}

(1) Qiu, G.; Knowles, R. R. "Rate-Driving Force Relationships in the Multisite-PCET Activation of Ketones." J. Am. Chem. Soc. 2019, 141, 2721-2730.

(2) Breslow, R.; Chu, W. "Thermodynamic Determination of pKa's of Weak Hydrocarbon Acids Using Electrochemical Reduction Data." J. Am. Chem. Soc. 1973, 95, 411

(3) Bordwell, F. G.; Cheng, J. P.; Harrelson, J. A. "Homolytic bond dissociation energies in solution from equilibrium acidity and electrochemical data." J. Am. Chem. Soc. 1988, 110, 1229

(4) Warren, J. J.; Tronic, T. A.; Mayer, J. M. "Thermochemistry of proton-coupled electron transfer reagents and its implications." Chem. Rev. 2010, 110, 6961

(5) Choi, G. J.; Knowles, R. R. Catalytic Alkene Carboaminations Enabled by Oxidative Proton-Coupled Electron Transfer. J. Am. Chem. Soc. 2015, 137, 9226.

(6) Cheng, J. P.; Zhao, Y. Y. "Homolytic Bond Cleavage Energies of the Acidic N-H Bonds in Dimethyl Sulfoxide Solution and Properties of the Corresponding Radicals and Radical Cations." Tetrahedron 1993, 49, 5267.

(7) Bordwell, F. G., Zhang, X. M., Satish, A. V., \& Cheng, J. P. “Assessment of the importance of changes in ground-state energies on the bond dissociation enthalpies of the $\mathrm{OH}$ bonds in phenols and the $\mathrm{SH}$ bonds in thiophenols." J. Am. Chem. Soc. 1994, 116, 6605-6610

(8) Dené s, F.; Pichowicz, M.; Povie, G.; Renaud, P. "Thiyl Radicals' in Organic Synthesis." Chem. Rev. $2014,114,2587-2693$

(9) dos Santos, Daniel JVA, Ana S. Newton, Raul Bernardino, and Rita C. Guedes. "Substituent effects on $\mathrm{O}-\mathrm{H}$ and $\mathrm{S}-\mathrm{H}$ bond dissociation enthalpies of disubstituted phenols and thiophenols." International Journal of Quantum Chemistry 2008, 108, 754-761.

(10) Miller, D. C.; Choi, G. J.; Orbe, H. S.; Knowles, R. R. Catalytic Olefin Hydroamidation Enabled by Proton-Coupled Electron Transfer. J. Am. Chem. Soc. 2015, 137, 13492-13495. 